Portland State University

PDXScholar

Dissertations and Theses

Dissertations and Theses

$1-1-2010$

\title{
Confirmation Bias and Related Errors
}

Geoffrey Ludlow Borthwick

Portland State University

Follow this and additional works at: https://pdxscholar.library.pdx.edu/open_access_etds Let us know how access to this document benefits you.

\section{Recommended Citation}

Borthwick, Geoffrey Ludlow, "Confirmation Bias and Related Errors" (2010). Dissertations and Theses. Paper 128.

https://doi.org/10.15760/etd.128

This Dissertation is brought to you for free and open access. It has been accepted for inclusion in Dissertations and Theses by an authorized administrator of PDXScholar. Please contact us if we can make this document more accessible: pdxscholar@pdx.edu. 


\section{Confirmation Bias and Related Errors}

by

Geoffrey Ludlow Borthwick

A dissertation submitted in partial fulfillment of the requirements for the degree of

Doctor of Philosophy

in

Systems Science: Psychology

Dissertation Committee:

Thomas Kindermann, Chair

Barry F. Anderson

Todd E. Bodner

Wayne Wakeland

John Settle

Portland State University

(C) 2010 


\begin{abstract}
This study attempted to replicate and extend the study of Doherty, Mynatt, Tweney, and Schiavo (1979), which introduced what is here called the Bayesian conditionals selection paradigm. The present study used this paradigm (and a script similar to that used by Doherty et al.) to explore confirmation bias and related errors that can appear in both search and integration in probability revision. Despite selection differences and weak manipulations, this study provided information relevant to four important questions.
\end{abstract}

First, by asking participants to estimate the values of the conditional probabilities they did not learn, this study was able to examine the use of "intuitive conditionals”. This study found evidence that participants used intuitive conditionals and that their intuitive conditionals were affected by the size of the actual conditionals.

Second, by examining both phases in the same study, this study became the first to look for inter-phase interactions. A strong correlation was found between the use of focal search strategies and focal integration strategies $(r=.81, \mathrm{p}<.001)$. However, when the sample was limited to participants who selected at least one probability conditioned on each hypothesis, the relationship was near random $(r=.02)$.

Third, this study was the first in the Bayesian conditionals selection paradigm to provide a basis for selecting information that would be expected to confirm rather than disconfirm the focal hypothesis. No support was found for predictor selection 
bias, but a nearly significant interaction was found between Attention and Motivation ( $p=.07$ ) in applying Bayes' theorem only to information favoring the focal hypothesis.

Fourth, this study was the first to have a single normative posterior probability, against which participants’ posterior probability estimates could be compared to yield a quantitative measure of confirmation bias. This permitted measuring the confirmation bias uniquely contributed by each phase, assuming the participant was normative in the other phase. Focused attention was found to increase confirmation bias in the integration phase $(p=.03)$ but not in the search phase.

These last three findings challenge the field's assumption that the search and integration phases can be examined separately, and call for reinterpretation of research done on only one phase. 
Table of Contents

Abstract............................................................... i

List of Tables............................................................ viii

List of Figures..................................................... xii

Introduction............................................................... 1

Literature Review .................................................. 10

Research Paradigms............................................ 10

Bayesian Conditional Selection Paradigm................ 11

Bayesian Data Sampling Studies ..................... 17

Wason Selection Task Studies........................... 20

Wason Rule-Discovery Task Studies..................... 22

Classification Studies ............................... 22

Covariation and Causation Studies ..................... 23

Dissonance Theory Studies .......................... 25

Consistent Findings Across Paradigms ............................... 26

Failure to use Base Rate Information. .................. 27

Searching for Less than Maximally Diagnostic Information 27

Searching for Information About or Associated With the

Focal Hypothesis .................................... 28

Integration of Non-Diagnostic Information ............... 28

Use of Averaging Integration Strategies. ............... 29

Causes and Cures of Biases ...................................... 29

Attention. .......................................... 29

Motivation. ......................................... 30

Timing-of-Feedback .............................. 30

Surprise. ...................................... 30

Problems with Current Research.................................... 31

The Present Study. .................................................. 36

Similarities to Doherty, et al. (1979)................... 37

Differences from Doherty, et al. (1979)................ 38

Hypotheses.................................................. 42

Expected Effects of Independent Variables...................... 43

Research Hypotheses......................................... 45

Predictor Selection Bias ............................... 47

Biased Bayesian Strategy in Integration ................ 47

Confirmation Bias ................................. 48 
Non-Independence of Phases ............................ 48

Exploratory Hypotheses. ................................... 49

Exploratory Search Process Hypotheses................. 51

Hypothesis Focus Bias ......................... 51

Pseudodiagnosticity.................................. 51

Focal Search Strategies.............................. 52

Comparing the Size of Hypothesis Focus and

Predictor Selection Biases ...................... 52

Exploratory Integration Process Hypotheses............... 53

Hypothesis-Focus Integration Bias................ 53

Predictor-Focus Integration Bias ................. 54

Pairs Used in Integration ........................ 54

Use of Biased Integration Strategies .............. 55

Phase Biases..................................... 55

Intuitive Conditionals............................ 57

Surprise Hypotheses ........................... 58

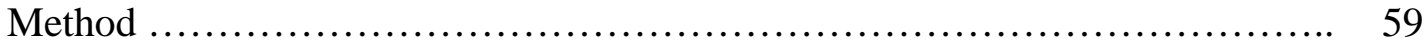

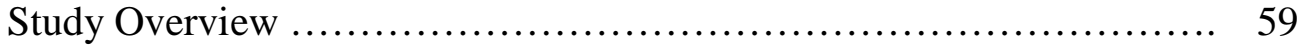

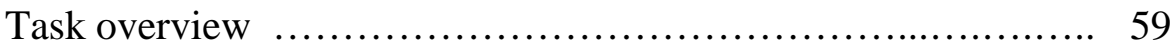

Measures overview............................................... 60

Concurrent Search Phase Measurements ................ 60

Concurrent Integration Phase Measurements .............. 60

Retrospective Measurements ........................... 60

Demographic Measurements ............................. 61

Participants......................................................... 61

Demographics ............................................ 61

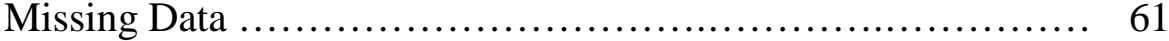

Experimental Design ............................................. 62

Structural Design ........................................... 62

Design Features Used to Ensure a Single Optimal Search and

Integration Strategy .......................................... 62

Theoretical Definitions of Independent Variables .................. 62

Theoretical Definitions of Dependent Variables .................... 64

Cell Sizes ........................................................... 66

Operational Definitions of Independent Variables ...................... 68

$\mathrm{IV}_{1}$ Attention Operational Definition .......................... 68

$\mathrm{IV}_{2}$ Motivation Operational Definition ........................... 73

$\mathrm{IV}_{3}$ Timing of Feedback Operational Definition ................... 74

$\mathrm{IV}_{4}$ Size of Conditionals Operational Definition .................... 75

Biasing Conditions Operational Definition ........................ 77

Operational Definitions of Direct Dependent Variables .................. 77 
Operational Definitions of Direct Dependent Search Phase

Variables

Operational Definitions of Direct Dependent Integration Phase

Variables

Information Usage Dependent Variables ................ 83

Operational Definitions of Derived Dependent Variables .............. 87

Operational Definition of $\mathrm{DV}_{4}$ : Search Strategy .................. 87

Bayesian Strategies ................................ 91

Non-Bayesian One-Category Strategies ................ 92

Non-Bayesian Two-Category Strategies ............... 92

Non-Bayesian Four-Category Strategy ................. 93

Unclassifiable Strategies ........................... 93

Classification of Search Strategies as Focal and Predictor

Biased ....................................... 93

Operational Definition of $\mathrm{DV}_{11}$ : Integration Strategy ............ 96

Mathematical Two-Island Bayesian Strategies............ 105

Mathematical Two-Island Non-Bayesian Strategies ....... 107

Mathematical One-Island Strategies................... 109

Mathematical Zero-Island Strategy...................... 111

Non-Mathematical Strategies ............................ 111

Classification of Integration Strategies as Focal and

Predictor Biased ...................................... 113

Operational Definition of $\mathrm{DV}_{6}$ : Search Confirmation Bias ........ 115

Operational Definition of $\mathrm{DV}_{12}$ : Integration Confirmation Bias ... 116

Operational Definition of $\mathrm{DV}_{13}$ : Sum-of-Phase-Biases ........... 119

Operational Definition of $\mathrm{DV}_{15}$ : Intuitive Conditionals .......... 120

Procedure ..................................................... 122

Recruitment and Experimental Setting ....................... 122

Instructions to the Participants ............................... 123

Assignment to Conditions ................................. 123

Experimental Control: Double Blind ........................ 123

Materials ...................................................... 124

Decision Problem Introduction. ............................. 125

The Attention and Motivation Manipulations Structure ... 125

Attention Manipulation ............................... 126

Motivation Manipulation ........................... 127

Use of Frequency Form of Data. ..................... 127

Direction and Diagnosticity of Hints. ................. 127

Information Search Phase Structure ......................... 128

Timing-of-Feedback Manipulation. ....................... 129

Size-of-Conditionals Manipulation...................... 130

Information Search Data Collection. ................... 130 
Integration Phase ........................................... 130

Post Hoc Questions ......................................... 131

Information Usage Dependent Variables. .................. 131

Questions for Inferring Integration Strategy ................ 131

Surprise Questions. .................................. 132

Intuitive Conditionals Question........................... 133

Demographic Variables. ..................................... 133

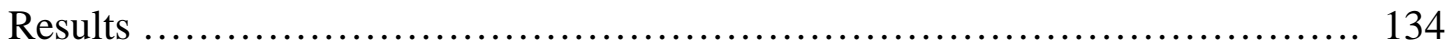

Preliminary Analyses ............................................ 134

Demographics ......................................... 134

Manipulation Checks ....................................... 134

Coherence Checks ............................................ 135

Problem with Random Assignment .............................. 135

Over-Biasing ............................................... 135

Search and Integration Strategy Classification Results ............. 136

Research Hypotheses.......................................... 140

Overall Testing Strategy for Research Hypotheses................. 140

Bonferroni Adjustment ................................. 140

Research Hypotheses Results. ................................. 141

Predictor Selection Bias .............................. 143

Biased Bayesian Strategy in Integration ................... 144

Confirmation Bias ..................................... 147

Non-Independence of Search and Integration Strategies .... 148

Exploratory Hypotheses.............................................. 151

Exploratory Search Phase Hypotheses . ........................... 153

Exploratory Integration Phase Hypotheses........................ 161

Phase Biases ................................................. 165

Intuitive Conditionals ......................................... 169

Surprise Hypotheses......................................... 174

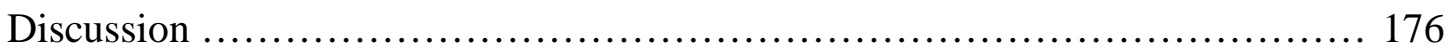

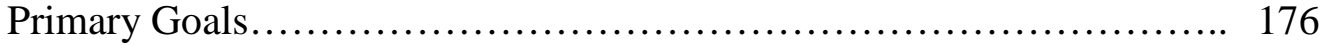

Results Summary ….............................................. 179

Results Categorized by Independent Variable ....................... 180

Attention Main Effects ....................................... 180

Motivation Main Effects .................................... 181

Motivation and Attention Interaction ........................... 181

Motivation and Attention Comparison .......................... 182

Timing of Feedback ......................................... 182

Size of Conditionals .......................................... 182

Results Categorized by Dependent Variable ......................... 183 
Search Phase ................................................ 183

Integration Phase .......................................... 184

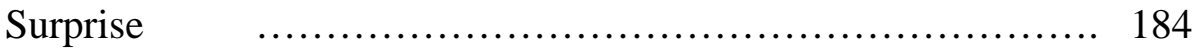

Intuitive Conditionals ......................................... 185

Search and Integration Strategies ........................... 187

Limitations ...................................................... 188

Limitations from the Scope of the Study ....................... 188

Limitations of Internal Validity from Manipulation Problems...... 189

Technical Limitation............................................... 192

Limitations of External Validity due to the Laboratory Setting..... 193

Implications ................................................... 195

Multi-Phase Research.......................................... 195

Technical Improvement for Future Laboratory Research........... 196

Future Research Topics ..................................... 197

Specific Future Studies ...................................... 200

Implications for Practice ..................................... 201

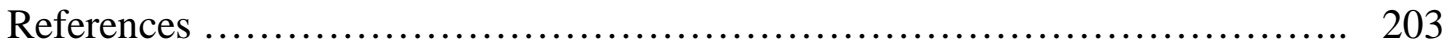

Appendices ................................................................. 214

Appendix A: Experimental Script .................................... 214

Appendix B: Instructions........................................ 222

Appendix C: Demographic Variables................................. 223

Appendix D: Informed Consent Form................................ 224

Appendix E: Pilot Study Integration Strategies........................ 225 
List of Tables

Table 1. Definition of Four Quadrants of Conditional Probabilities ............. 5

Table 2. Examples of the Biases Using Quadrant Definitions from Table 1 ....... 6

Table 3. Definition of Terms .............................................. 8

Table 4. Bayesian Conditional Selection Example ............................ 18

Table 5. Bayesian Data Sampling Example ............................... 19

Table 6. Studies by Paradigm and Decision Phases Examined $\ldots . . \ldots \ldots \ldots \ldots \ldots . . . . . .32$

Table 7. The Types of Conditional Probabilities in Doherty, et al.’s (1979) Study 40

Table 8. The Types of Conditional Probabilities in the Present Study............ 41

Table 9. Research Hypotheses and their Associated Independent and Dependent Variables 46

Table 10. Exploratory Hypotheses and their Associated Independent and Dependent Variables ............................................. 50

Table 11. Participants Completing each Phase in each Condition ............... 67

Table 12. Description of the Four Quadrants of Conditional Probabilities ........ 78

Table 13. Coding for Hypothesis Focus Bias................................ 80

Table 14. Coding for Predictor Selection Bias ............................ 80

Table 15. Coding for Biased Usage of Hints ............................... 84

Table 16. Coding for Hypothesis-Focus Integration Bias ...................... 85

Table 17. Coding for Predictor-Focus Integration Bias. ..................... 86

Table 18. Coding of Search Strategies as Focal and Predictor Biased ............ 95

Table 19. Exceptions to the Two Main Criteria for Classification to an Integration Strategy .............................................. 104 
Table 20. Example used for all Mathematical Strategies

Table 21. Coding of Integration Strategies as Focal and Predictor Biased

Table 22. Posterior Produced by Optimal Search and Each Integration Strategy ... 118

Table 23. Levels of the Four Independent Variables

Table 24. Order of Parts of Manipulation of Attention and Motivation Independent Variables 126

Table 25. Frequency of Search Strategies 138

Table 26. Frequency of Integration Strategies 139

Table 27. Research Hypotheses Results: Independent and Dependent Variables Involved

Table 28. The Effects of the Independent Variables on Predictor Selection Bias

Table 29. The Effects of the Independent Variables on Bias Bayesian Strategy...

Table 30. Post Hoc Orthogonal Contrasts Exploring Over-Biasing Effect on use of Biased Bayesian Strategies

Table 31. The Effects of the Independent Variables on Confirmation Bias

Table 32. Cross Tabulation of Search and Integration Strategies by Focal Island

Table 33. Cross Tabulation of Search and Integration Strategies by Conditionals Selected

Table 34. Exploratory Hypotheses Results: Independent and Dependent Variables Involved

Table 35. The Effects of the Independent Variables on Hypothesis Focus Bias ... 154

Table 36. Orthogonal Contrasts on Over-Biasing Effect on Hypothesis Focus Bias 154

Table 37. Descriptive Statistics on Over-Biasing Effect on Hypothesis Focus Bias 155 
Table 38. The Effects of the Independent Variables on Pseudodiagnosticity ..... 156

Table 39. The Effects of the Independent Variables on Focal Search Strategies .. 157

Table 40. Orthogonal Contrasts on Over-Biasing Effect on Focal Search Strategies

Table 41. Descriptive Statistics on Over-Biasing Effect on Focal Search Strategies

Table 42. The Effects of the Independent Variables on Hypothesis-Focus Integration Bias

Table 43. The Effects of the Independent Variables on Predictor-Focus Integration Bias

Table 44. The Effects of the Independent Variables on Pairs Used in Integration

Table 45. The Effects of the Independent Variables on Use of Biased Integration Strategies

Table 46. Orthogonal Contrasts on Over-Biasing Effect on Use of Biased Integration Strategies

Table 47. Descriptive Statistics on Over-Biasing Effect on Use of Biased Integration Strategies

Table 48. The Effects of the Independent Variables on Search Confirmation Bias

Table 49. The Effects of the Independent Variables on Integration Confirmation Bias

Table 50. Orthogonal Contrasts on Over-Biasing Effect on Integration Confirmation Bias

Table 51. Descriptive Statistics on Over-Biasing Effect on Integration Confirmation Bias

Table 52. Comparison Accuracy of Models in Predicting Actual Posterior Estimates 
Table 53. Descriptive Statistics for Surprised and Not-Surprised Participants in Motivated and / or Focused Conditions ............................... 
List of Figures

Figure 1. Classification of Search Strategies by the Number of Cells Used and the Use of Matched Pairs.

Figure 2. Categorization of Integration Strategies by the Information and Math Used

Figure 3. Attention by Motivation Interaction on Use of Biased Bayesian Integration Strategies.

Figure 4. Attention by Size-of-Conditionals Interaction on Use of Biased

Bayesian Integration Strategies

Figure 5. Attention by Motivation Interaction on Hypothesis Focus Bias

Figure 6. Attention by Motivation Interaction on Use of Focal Search Strategies

Figure 7. Attention by Size of Conditionals Interaction on Use of Focal Search Strategies

Figure 8. Motivation by Attention on Integration Confirmation Bias 
Introduction 1

\section{Introduction}

Decision making is defined as "selecting and committing oneself to a course of action” (Anderson, Deane, Hammond, McClelland, and Shanteau, 1981, p. 73).

Studies have shown people have trouble with risky (probabilistic) decision making. One reason for this difficulty is difficulty with probability revision, the changing of a belief on the basis of new information. Nickerson (2004) states that, "Everybody thinks probabilistically, whether knowingly or not” (p. ix). Probabilistic decision making is both required by daily life (e.g., estimating the probability of finding a parking space in various locations) and demonstrated to be flawed in non-experts and occasionally in experts (Tversky \& Kahneman, 1971). As Persi Diaconis said in commenting on the topic, "Our brains are just not wired to do probability problems very well, so I'm not surprised there were mistakes.” (Tierney, 1991, p. A1).

For example, the importance of probabilistic reasoning in decision-making is highlighted by the trend away from defined benefit plans (social security, defined benefit private pensions) towards individually managed retirement plans (IRAs, 401k, potential private social security accounts). This trend will require more Americans to make important probabilistic decisions.

The decision making process is commonly divided into two phases, information search (where the decision maker gathers information to use in the decision) and information integration (where the decision maker combines information to make a decision). Non-expert decision makers suffer from many errors in both phases. One class of errors that has been repeatedly demonstrated is a variety 
Introduction 2

of common biases that are associated with the existence of a preferred or focal hypothesis. These biases have been extensively researched (115,073 hits on the PsychInfo Data base on 11/2/05) and demonstrated across a variety of paradigms and cover stories. These biases are usually grouped together and commonly called "confirmation bias" (or some variant of the term).

Unfortunately, this term has been not been consistently defined. Confirmation bias has been used as a catchall for virtually any bias related to decision-making that involves some form of focal hypothesis bias in selecting, remembering, or interpreting information or in judging the likelihood of the focal hypothesis. This label has covered both motivated and unmotivated biases and both those that occur in the information search phase and in the integration phase. The various definitions have included: a) biases in the search phase favoring information about the focal hypothesis (Doherty, Mynatt, Tweney, \& Schiavo, 1979); b) limitations of the range of information collected to that which is consistent with the focal hypothesis (Trope \& Bassok 1984); c) preference for the collection of information that is expected to be supportive to the focal hypothesis (Jonas, Schulz, Frey, \& Thelen, 2001); d) biases caused by differential rates of memory for favorable and unfavorable evidence (Perkins, Farady, \& Bushey, 1991); and e) interpretation of evidence in ways that support the focal hypothesis. (Kelley, 1950). These five terms each refer to a different phenomenon, of which only the last four will always result in a confirmatory effect.

Because the term “confirmation bias” has been used to describe a variety of phenomena, the use of the term has led to difficulty in comparing results across 
Introduction 3

paradigms and researchers. This has led to the recommendation that the term be retired (Fischhoff \& Beyth-Marom, 1983). However, the position taken in this paper is that it would be better to define the term clearly than to retire it. In this paper, "confirmation bias” will be used to refer to any bias that actually results in a confirming effect (i.e., an increase in the estimated probability of the focal hypothesis above the normative posterior probability). Confirmation bias as thus defined is a resulting effect of other cognitive processes. These may be search or integration processes, which will be referred to respectively as "Search Confirmation Bias” and “Integration Confirmation Bias”. This definition works for all paradigms that result in judgment or choice (Bayesian Conditionals Selection Paradigm, Bayesian Data Sampling Studies, Covariation and Causation Studies, and Dissonance Theory Studies, see Research Paradigms for a description of these paradigms).

This paper will use three terms that can be confused with confirmation bias and with each other, and which therefore need to be clarified. These are the search phase biases of “pseudodiagnosticity”, “hypothesis focus bias”, and “predictor selection bias”.

“Pseudodiagnosticity” is here accepted as defined by Doherty, et al. (1979) in a study examining participants' search-phase behavior. They used the term to describe the search phase phenomenon of participants selecting only one member of a matching pair of conditional probabilities. A matching pair is defined as two probabilities both relevant to the same datum with one conditioned on each of the hypotheses [e.g. 
selecting both $p\left(\mathrm{D}_{\mathrm{i}} \mid \mathrm{H}_{1}\right)$ and $p\left(\mathrm{D}_{\mathrm{i}} \mid \mathrm{H}_{2}\right)$ ]. (See Tables 1 and 2). Matching pairs are necessary for the application of Bayes' Theorem.

$$
p\left(\mathrm{H}_{1} \mid \mathrm{D}\right)=\frac{p\left(\mathrm{H}_{1}\right) * p\left(\mathrm{D} \mid \mathrm{H}_{1}\right)}{p\left(\mathrm{H}_{1}\right) * p\left(\mathrm{D} \mid \mathrm{H}_{1}\right)+p\left(\mathrm{H}_{2}\right) * p\left(\mathrm{D} \mid \mathrm{H}_{2}\right)}
$$

Bayes' Theorem is the correct method for revising probability estimates on the basis of new information. $\left[p\left(\mathrm{H}_{1} \mid \mathrm{D}\right)\right.$ is the probability of hypothesis 1 given the new data, $p\left(\mathrm{H}_{1}\right.$ or 2 ) is the original probability of hypothesis 1 (or 2$), p\left(\mathrm{D} \mid \mathrm{H}_{1}\right.$ or 2$)$ is the probability of the data given hypothesis 1 (or 2)]. 
Introduction 5

Table 1

Definition of Four Quadrants of Conditional Probabilities

\begin{tabular}{llll}
\hline $\begin{array}{l}\text { Expected direction } \\
\text { of dimension’s }\end{array}$ & Data & Probabilities & Probabilities conditioned \\
diagnosticity & & conditioned on the & on the alternative \\
& focal hypothesis $\left(\mathrm{H}_{1}\right)$ & hypothesis $\left(\mathrm{H}_{2}\right)$ \\
& (Selected) (Hit Rate) & (Selected) (False Alarm \\
& Rate)
\end{tabular}

\begin{tabular}{ll|l|l|}
\hline $\begin{array}{l}\text { Predictor expected } \\
\text { to favor focal }\end{array}$ & $\mathrm{D}_{1}$ & Quadrant 1: $p\left(\mathrm{D}_{1} \mid \mathrm{H}_{1}\right)$ & Quadrant 3: $p\left(\mathrm{D}_{1} \mid \mathrm{H}_{2}\right)$ \\
$\begin{array}{l}\text { hypothesis } \\
\text { Predictor expected } \quad \mathrm{D}_{2}\end{array}$ & Quadrant 2: $p\left(\mathrm{D}_{2} \mid \mathrm{H}_{1}\right)$ & Quadrant 4: $p\left(\mathrm{D}_{2} \mid \mathrm{H}_{2}\right)$ \\
\cline { 3 - 4 } \\
to favor non-focal \\
hypothesis
\end{tabular}

Note: Data would be in the form of the urn has two handles. The conditional probabilities in quadrants 1 and 2 would be in the form of the percentage of urns from Shell with two handles. 
Introduction 6

Table 2

Examples of the Biases Using Quadrant Definitions from Table 1

\begin{tabular}{lll}
\hline Name of Bias & Definition of Bias & Example from Table 1 \\
\hline Pseudodiagnosticity & Selecting only one member of a & Selecting $p\left(\mathrm{D}_{\mathrm{x}} \mid \mathrm{H}_{1}\right)$ \\
& matching pair & without $p\left(\mathrm{D}_{\mathrm{x}} \mid \mathrm{H}_{2}\right)$ \\
Hypothesis focus & Selecting more probabilities & Selecting more \\
bias & conditioned on the focal & probabilities from \\
& hypothesis than the non-focal & Quadrants 1 \& 2 \\
& hypothesis & \\
Predictor selection & Selecting more probabilities from & Selecting more \\
bias & dimensions expected to favor the & probabilities from \\
& focal hypothesis & Quadrants 1 \& 3
\end{tabular}

Doherty, et al. (1979) found that participants tend to choose disproportionate number of probabilities conditioned on the focal hypothesis (hereafter symbolically represented as $\mathrm{H}_{1}$, with the alternative hypothesis represented as $\mathrm{H}_{2}$ ). [This can also be stated as choosing more $p\left(\mathrm{D} \mid \mathrm{H}_{1}\right)$ than $\left.p\left(\mathrm{D} \mid \mathrm{H}_{2}\right)\right]$. (See Tables 1 and 2). Doherty labeled this bias “confirmation bias”. However, in order to avoid confusion, in this paper we will substitute what we believe to be the more precise term "hypothesis focus bias". Pseudodiagnosticity is a necessary, but not sufficient, condition for hypothesis focus 
Introduction 7

bias. Participants must be pseudodiagnostic in order to show a hypothesis focus bias, but they can be pseudodiagnostic without showing hypothesis focus bias (by selecting non-matched conditionals that are evenly split between the two hypotheses or that are more often conditioned on the alternative hypothesis).

A key point stressed in this paper, which was first noted for hypothesis focus bias by Fischhoff and Beyth-Marom, (1983) is that neither of these search phase biases alone or in combination necessarily results in making confirmation more likely (that is, showing what is here called confirmation bias). The extent to which these biases have a confirming effect would depend upon the conditionals selected and the integration strategy utilized, as first noted by Snyder and Swan (1978). For example, use of a hypothesis focus bias or pseudodiagnosticity would not result in confirmation bias in combination with a Bayesian integration strategy and, in fact, would result in no revision at all, due to the lack of matching pairs.

The third term that can be confused with confirmation bias, "predictor selection bias”, is the preference for information from a variable that is expected to confirm a focal hypothesis. [This can also be stated as selecting more $p(\mathrm{D}$ expected to favor ${ }_{\mathrm{H} 1} \mid \mathrm{H}_{1}$ or 2 ) than $p\left(\mathrm{D}\right.$ expected to favor $\mathrm{H}_{2} \mid \mathrm{H}_{1}$ or 2$\left.)\right]$. (See Table 1 for a description of a design that would enable examination of predictor selection bias and Table 2 for examples of these conditional probabilities). For this bias to operate there must be some basis for expecting some variable or variables to favor the focal hypothesis more than other variables (See Table 3 for definitions). This bias can exist with the selection of either matched or unmatched conditional probabilities. 
Introduction 8

Table 3

Definition of the Terms

\begin{tabular}{|c|c|c|c|c|}
\hline Phase & Bias Name & Page & Original Name & Definition \\
\hline \multirow{5}{*}{$\begin{array}{l}\text { Search } \\
\text { Phase } \\
\text { Only } \\
\text { (Selecting) }\end{array}$} & Search & $2-3$ & & $p(\mathrm{H} \mid \mathrm{D})$ greater than \\
\hline & Confirmation Bias & & & normative from search phase \\
\hline & Pseudodiagnosticity & 3 & & $\begin{array}{l}\text { Not selecting both } \\
\text { conditional probabilities on a } \\
\text { dimension }\left[p\left(\mathrm{D}_{1} \mid \mathrm{H}_{2}\right) \&\right. \\
p\left(\mathrm{D}_{1} \mid \mathrm{H}_{1}\right)\end{array}$ \\
\hline & $\begin{array}{l}\text { Hypothesis Focus } \\
\text { Bias }\end{array}$ & 4 & $\begin{array}{l}\text { Confirmation } \\
\text { Bias (Doherty, } \\
\text { et al., 1979) }\end{array}$ & $\begin{array}{l}\text { Selecting more conditional } \\
\text { probabilities conditioned on } \\
\text { the focal hypothesis } p\left(\mathrm{D} \mid \mathrm{H}_{2}\right) \\
\text { than the alternative } \\
\text { hypothesis } p\left(\mathrm{D} \mid \mathrm{H}_{1}\right)\end{array}$ \\
\hline & $\begin{array}{l}\text { Predictor Selection } \\
\text { Bias }\end{array}$ & 4 & $\begin{array}{l}\text { Confirmation } \\
\text { Bias (Jonas, } \\
\text { Schulz-Hardt, } \\
\text { Frey, \& } \\
\text { Thelen, 2001) }\end{array}$ & $\begin{array}{l}\text { Selecting more conditional } \\
\text { probabilities from } \\
\text { dimensions that are expected } \\
\text { to support the focal } \\
\text { hypothesis }\end{array}$ \\
\hline \multirow{4}{*}{$\begin{array}{l}\text { Integration } \\
\text { Phase } \\
\text { Only } \\
\text { (Using) }\end{array}$} & $\begin{array}{l}\text { Integration } \\
\text { Confirmation Bias }\end{array}$ & $2-3$ & & $\begin{array}{l}p(\mathrm{H} \mid \mathrm{D}) \text { greater than } \\
\text { normative from integration } \\
\text { phase }\end{array}$ \\
\hline & $\begin{array}{l}\text { Pseudodiagnostic } \\
\text { Integration }\end{array}$ & $4-5$ & & $\begin{array}{l}\text { Not using both conditional } \\
\text { probabilities on a dimension } \\
{\left[p\left(\mathrm{D}_{1} \mid \mathrm{H}_{2}\right) \& p\left(\mathrm{D}_{1} \mid \mathrm{H}_{1}\right)\right.}\end{array}$ \\
\hline & $\begin{array}{l}\text { Hypothesis-Focus } \\
\text { Integration Bias }\end{array}$ & $4-5$ & & $\begin{array}{l}\text { Using more conditional } \\
\text { probabilities conditioned on } \\
\text { the focal hypothesis } p\left(\mathrm{D} \mid \mathrm{H}_{2}\right) \\
\text { than the alternative } \\
\text { hypothesis } p\left(\mathrm{D} \mid \mathrm{H}_{1}\right)\end{array}$ \\
\hline & $\begin{array}{l}\text { Predictor Selection } \\
\text { Integration Bias }\end{array}$ & $4-5$ & & $\begin{array}{l}\text { Using more conditional } \\
\text { probabilities from } \\
\text { dimensions that are expected } \\
\text { to support the focal } \\
\text { hypothesis }\end{array}$ \\
\hline \multirow[t]{2}{*}{$\begin{array}{l}\text { Both } \\
\text { Phases }\end{array}$} & Confirmation Bias & 2 & & $\begin{array}{l}p(\mathrm{H} \mid \mathrm{D}) \text { greater than } \\
\text { normative from all causes }\end{array}$ \\
\hline & Base Rate Neglect & 26 & & $\begin{array}{l}\text { Ignoring or underweighting } \\
p(\mathrm{H})\end{array}$ \\
\hline
\end{tabular}


Introduction 9

These three search phase biases (pseudodiagnosticity, hypothesis focus bias, and predictor selection bias) all have corresponding integration phase biases, which here will be referred to, respectively, as "pseudodiagnostic integration”, "HypothesisFocus Integration Bias”, and “predictor selection integration bias”. (The reason "pseudodiagnostic integration" is not referred to as "pseudodiagnostic integration bias" is because this error does not imply a direction in the participants' departure from normative). All three of these integration phase biases need to be studied in addition to their search phase counterparts, because information that is selected is not necessarily used.

Pseudodiagnostic integration is the use in integration of unmatched conditional probabilities. Hypothesis-Focus Integration Bias is the disproportionate use of probabilities in integration that are conditioned on the focal hypothesis. (As was the case in the search phase, pseudodiagnostic integration bias is required for the possibility of Hypothesis-Focus Integration Bias). Predictor selection integration bias is a bias towards using predictors in integration that are expected to support the focal hypothesis. This last definition is problematic in the abstract, because some predictors could be expected in the search phase to support the focal hypothesis but in the integration phase, on the basis of the data now in hand, to support the alternative hypothesis. This problem is avoided in this study by making all predictors either consistently favor or consistently oppose the focal hypothesis. 
Literature Review 10

\section{Literature Review}

\section{Research Paradigms}

Research on search and integration biases that can result in confirmation bias has been conducted under at least the following seven paradigms: Bayesian conditional selection studies, Bayesian data sampling studies, Wason selection task studies, Wason rule-discovery task studies, classification studies, covariation and causation studies, and dissonance theory studies.

In the Bayesian conditional selection studies, participants are asked to select and/or incorporate conditional probability information to make revised probability estimates. This paradigm usually, but not always, focuses on the search phase. In the Bayesian data sampling studies, participants are asked which population a sample of data was drawn from. These studies usually focus on the integration phase. The Wason four-card selection task requires participants to select the information (cards) necessary to test a logical rule and looks exclusively at the search phase. The Wason rule-discovery task requires participants to seek information to assist in their formulating and evaluating hypotheses. This task is the most open and involves hypothesis creation, information search, and information integration. The classification studies are similar in logical structure to the rule-discovery task. As in that paradigm, participants must think up questions to test a hypothesis, but this time about category membership. The covariation and causation paradigm asks participants to select or evaluate information necessary to make a decision regarding the degree of 
covariation or causation. These studies, with one exception, examine only the integration phase.

Finally, the dissonance theory research paradigm has participants make an initial decision and then presents them with the opportunity to select information that would support or oppose this decision. These studies focus on the search phase.

Bayesian conditional selection paradigm. Research under this paradigm presents participants with cover stories that require a probability revision. Typically, participants are given the opportunity to select some but not all of the available information in the form of conditional probabilities. These probabilities are conditioned on two possible hypotheses, one of which has already been determined, on some basis, to be the favored or focal hypothesis. As previously noted, the correct way to incorporate new information into a probability estimate involves the application of Bayes' Theorem. As applied to Bayesian conditional selection studies Bayes' theorem requires a prior probability estimate (typically provided to participants as a base rate), one or more items of data (also usually provided to participants) and a matching pair of conditional probabilities for the data. Participants usually chose which conditional probabilities to be informed of, after being informed of the related data. The focus of this paradigm is on the extent to which participants fail to select matching pairs (exhibit pseudodiagnosticity) and the extent to which these unmatched probabilities are conditioned on the focal hypothesis (exhibit hypothesis focus bias).

The groundbreaking study using a Bayesian conditional selection paradigm to study search biases was Doherty, et al. (1979), to which the present study is closely 
related. They used an ocean archeology cover story to present participants with an information search problem. Their experiment involved two steps. In the first step, they presented participants with the problem (deciding which island an artifact was made on), the base rate for each hypothesis, and descriptive data about the artifact. Next, participants were required to choose two conditional probabilities from four relevant to the descriptive data. Last, each participant was asked which hypothesis was now more likely. This hypothesis was taken to be that participant's focal hypothesis.

Half the participants were provided an equal base rate (odds ratio $p(\mathrm{~h}) \mid p(-\mathrm{h})=$ 1:1), and half were provided a base rate that favored one of the hypotheses (odds ratio of 10:1). This 10:1 ratio was designed to be so strong that the estimate as to which hypothesis was more likely would not be changed even by either of the data favoring the less likely hypothesis (with diagnostic ratios of 1:5).

The unequal base rate group produced two main findings. The first finding was that the participants were pseudodiagnostic, with 49 of the 64 participants choosing one conditional probability from each dimension, rather that the two required to form a diagnostic pair. Therefore, these participants did not have sufficient information to revise their probability estimates using Bayes’ Theorem. The second finding was that 52 of the 64 participants indicated as more likely the hypothesis favored by the data rather than the base rate. This choice was incorrect for both those who chose diagnostically (for whom the base rate was normatively stronger than the data) and those who chose pseudodiagnostically (who had no normatively relevant evidence other than the base rate). The authors stated that this effect was an indication of base 
Literature Review 13

rate neglect. Doherty, et al. (1979) did not report the degree to which the participants who demonstrated the first error (pseudodiagnosticity) were same as those who demonstrated the second error (base rate neglect).

In the second step, Doherty, et al. (1979) asked participants to select six conditional probabilities from a set of twelve, to assist in making a second probability revision. Six of these probabilities were conditioned on the focal hypothesis and six on the alternative hypothesis, forming six matching pairs. Doherty, et al. examined this second step in terms of both pseudodiagnosticity and hypothesis focus bias, and found evidence for both search errors. First, 110 of the 121 participants failed to choose three matched pairs, thus demonstrating some degree of pseudodiagnosticity. Because one must be pseudodiagnostic to demonstrate hypothesis focus bias only the 110 pseudodiagnostic participants were examined for hypothesis focus bias. Each of these 110 pseudodiagnostic participants showed one of three possible patterns: a) choosing probabilities conditioned evenly on the two hypotheses (50), b) choosing more probabilities conditioned of the alternative hypothesis (9), or c) choosing more probabilities conditioned of the focal hypothesis (51). The propensity of participants to choose conditional probabilities conditioned on the focal hypothesis when they did not choose evenly (51 to 9), indicates hypothesis focus bias (labeled “confirmation bias” by Doherty, et al.). They interpreted this finding as a "strong bias to confirm”, despite the fact that any actual confirmatory effect would be dependent on the participant's integration strategy. 
Doherty, Schiavo, Tweeny, and Mynatt (1981) conducted an experiment to determine whether people can be induced to think diagnostically. They based their experiment on the first step of the Doherty, et al. (1979) experiment, (specifically the equal base rate condition), where participants had been asked to choose two conditional probabilities from four that formed two matched pairs that favored one of the hypotheses. They made four changes from the original experiment that were intended to help participants think diagnostically.

Three of these changes were made for all participants: a) presenting the problem four times, using different cover stories each time, b) providing accurate feedback after each story as to which hypothesis was more likely, and c) making all conditional probabilities above $50 \%$. The fourth change was to give the half the participants a third conditional probability from the original four. For participants who chose non-diagnostically, this third conditional probability necessarily completed a diagnostic pair whose diagnostic direction favored the second hypothesis. This diagnostic pair would contradict the conclusion that would likely have been drawn by participants who choose both probabilities conditioned on the first hypothesis. In this case, the two conditional probabilities would both be above $50 \%$ and both would likely be interpreted as supportive of the first hypothesis.

Following the initial study, Doherty, et al. (1981) found that participants who did not receive the third conditional probability did marginally improve their rate of diagnostic search over the four trials. However, those who received the third conditional probability nearly tripled their rate of diagnostic search over the four trials. 
This suggests that participants have the capacity to think diagnostically when the situation presents them with diagnostic information.

Two years later, Beyth-Marom and Fischhoff (1983) published a series of studies that examined participants' search phase behavior. In the first study, the authors asked participants whether $p\left(\mathrm{H}_{1}\right)$ (the base rate), $p\left(\mathrm{D} \mid \mathrm{H}_{1}\right)$ (the hit rate), and $p\left(\mathrm{D} \mid \mathrm{H}_{2}\right)$ (the false alarm rate) are relevant to a probability revision. Approximately $90 \%$ of participants reported that $p\left(\mathrm{H}_{1}\right)$ and $p\left(\mathrm{D} \mid \mathrm{H}_{1}\right)$ were relevant, but only $50 \%$ reported that $p\left(\mathrm{D} \mid \mathrm{H}_{2}\right)$ was relevant. This was true regardless of whether the alternative hypothesis was stated as a single possibility, multiple possibilities, or only an implied other. This rate of interest in the alternative hypothesis declined even further when subjects were told to stick to relevant information only. In a second study, BeythMarom and Fischhoff asked participants why each type of information was relevant. Most (69\%) of those who thought $p\left(\mathrm{D} \mid \mathrm{H}_{2}\right)$ was relevant, saw it as being relevant in order to evaluate the alternative hypothesis directly $\left(\mathrm{H}_{2}\right)$, rather than to make a diagnostic comparison with $p\left(\mathrm{D} \mid \mathrm{H}_{1}\right)$. In a third study, Beyth-Marom and Fischhoff asked participants to make a revision with either diagnostic information (non-equal $p\left(\mathrm{D} \mid \mathrm{H}_{1}\right)$ and $p\left(\mathrm{D} \mid \mathrm{H}_{2}\right)$ ) or non-diagnostic (equal $p\left(\mathrm{D} \mid \mathrm{H}_{1}\right)$ and $p\left(\mathrm{D} \mid \mathrm{H}_{2}\right)$ ). Beyth-Marom and Fischhoff found that participants were more likely to revise on the basis of diagnostic information than non-diagnostic, and to revise in the correct direction. However, there was some evidence of base rate neglect, use of averaging strategies, and use of non-diagnostic information. Therefore, Beyth-Marom and Fischhoff 
concluded that, while not perfect, people are better at using information than at seeking it out.

Fischhoff and Bar-Hillel (1984) examined the integration phase using a between-subjects design. Across the cover stories, they varied the representativeness of the data. Next, they provided conditional probabilities for these data (i.e., there was no search phase). They found evidence of base rate neglect, which was stronger when representativeness of the data was high, as well as the use of averaging integration strategies.

Ofir (1988) used a series of between-subjects studies to examine integration strategies. Information was provided to participants (i.e., there was no search phase); and the base rate, the hit rate and the false alarm rate were all manipulated. Ofir had two findings. The first was that his participants did not exhibit base rate neglect. The second finding was that participants would use the false alarm rate data $\left[p\left(\mathrm{D} \mid \mathrm{H}_{2}\right)\right]$ only if the base rate and the hit rate $\left[p\left(\mathrm{D} \mid \mathrm{H}_{1}\right)\right]$ were on different sides of .5 and therefore psychologically inconsistent. This process appears similar to that identified by Smith, Schoben and Rips (1974), in which thinking becomes more precise only when necessary.

Mynatt, Doherty and Dragon (1993) examined participants’ search choices (but not their integration phase) on two logically identical problems which were worded differently. One problem was worded as a choice between two actions, and the second, as a comparative evaluation between two hypotheses. They provided the participants with a single hit rate and the choice to gather one more conditional 
probability from among a second hit rate and one of the two corresponding false alarm rates.

Mynatt, Doherty and Dragon’s (1993) principal finding was that participants were both less hypothesis focused and less pseudodiagnostic (i.e., more likely to select the matching false alarm rate) when choosing between actions than when evaluating hypotheses. Mynatt, et al. theorized that this difference was due to the outcome values being intrinsic to the evaluation of action choices but extrinsic to hypothesis evaluation, a difference that is more encouraging of comparison in action choices. This difference between action choices and hypothesis evaluation supports the conclusion that hypothesis focus bias is not an artifact of grammar or logical structure.

A second finding of this study was that participants in the inference condition were more likely to choose diagnostically when the provided probability conditioned on the focal hypothesis was below .5. The authors theorized that the low value of this probability switched participants’ focus to the alternative hypothesis.

Bayesian data sampling studies. The Bayesian data sampling studies use the same Bayesian framework but differ from the Bayesian conditional selection studies in that the participants in the Bayesian data sampling studies are given the conditional probabilities and must select data, while those in the Bayesian conditional selection studies are given the data and must select conditional probabilities.

The same situation can be presented to participants using either Bayesian paradigm. The Bayesian conditional selection paradigm might, for example, present participants with two kinds of data (two handles, and picture of a horse) and ask them 
to choose among four conditional probabilities to use in revising their probability estimate (see Table 4).

Table 4

Bayesian Conditional Selection Example

\begin{tabular}{lll}
\hline Feature of urn in & Percentage of Urns made & Percentage of Urns made \\
question & on Shell Island that have & on Coral Island that have \\
& feature & feature \\
\hline Urn has two handles & ? \% have two handles & ? \% have two handles \\
Urn has picture of a & ? \% have a horse & ? \% have a horse \\
horse & & \\
\hline
\end{tabular}

The Bayesian data sampling paradigm might, for example, present participants with four conditional probabilities and ask the participants to select which (of the two) kinds of data to use in revising their probability estimate (see Table 5). 
Table 5

Bayesian Data Sampling Example

\begin{tabular}{|c|c|c|}
\hline Feature of urn in & Percentage of Urns made & Percentage of Urns made \\
\hline \multirow[t]{2}{*}{ question } & on Shell Island that have & on Coral Island that have \\
\hline & feature & two handles \\
\hline \multirow[t]{2}{*}{ Two or One Handle(s)? } & $80 \%$ have two handles & $40 \%$ have two handles \\
\hline & - & \\
\hline \multirow[t]{2}{*}{ Picture of a horse? } & $30 \%$ have a picture of a & $60 \%$ have a picture of a \\
\hline & horse & horse \\
\hline
\end{tabular}

Phillips \& Edwards (1966) conducted the ground breaking study using this paradigm. Their focus was on the integration phase and examined how participants used the data that were provided (i.e., no search phase). They found that participants tended to under-adjust for the new information, an effect they labeled "conservatism.” (See Slovic \& Lichtenstien, 1971 for a review of this and the other early studies on conservatism).

Mynatt, Doherty and Sullivan (1991) conducted experiments on information search using the Bayesian data sampling paradigm. They presented participants with the decision as to which of two populations a sample had been drawn from. Participants were told that the two population proportions were 70:30 and 30:70 and that the proportion in the sample was 7:5. Participants were then presented an opportunity to draw another sample from either the population from which the first 
Literature Review 20

sample had been drawn or from the other population. Similar to the findings using the Bayesian conditional selection methodology, participants preferred to gather information repeatedly about the focal hypothesis. This bias appears whether gathering data or selecting conditional probabilities. This study collected no integration phase information.

Trautman and Shanteau (1977) found that non-diagnostic information caused participants to move towards a 50\%-50\% estimate of the probability that the data probability came from one of two sources. Nisbett, Zukier, and Lemley, (1981) replicated this finding, now called the "dilution effect”. This effect is non-normative, and usually anti-confirmatory, diluting the impact of other information. Kemmelmeier (2004) manipulated the instructions in order to examine the dilution effect in the integration phase. Half of the participants were instructed to identify non-diagnostic information before integrating. The other half of the participants were instructed to identify non-diagnostic information and completely black it out with a marker before integrating. The participants who blacked out the non-diagnostic information did not exhibit the dilution effect. However, those who did not black it out exhibited the dilution effect despite correctly identifying the non-diagnostic information.

Wason selection task studies. Wason (1966) introduced a new information search paradigm with his four-card problem. In this paradigm, participants are presented with four cards. They are told that each card has a number on one side and a letter on the other. The four cards are displayed with one of each possibility showing: even, odd, consonant and vowel. Participants are asked to indicate which cards must 
Literature Review 21

be turned over to test the rule that all the cards that have an even number on one side have a vowel on the other ("If E then V", more generally and commonly expressed as the conditional rule, "If P Then Q").

Wason found that participants most commonly asked to turn over two cards, the $\mathrm{E}$ and the $\mathrm{V}$ cards, a pattern which he labeled "verification bias". This pattern is similar to hypothesis focus, gathering information about the focal hypothesis. If the focal hypothesis is taken to be "If $\mathrm{E}$ then V", then participants gather information about the $\mathrm{E}$ and $\mathrm{V}$ cards, elements of the statement of the focal hypothesis.

Wason and Johnson-Laird (1970) replicated Wason (1966) and additionally found that instructions to evaluate the effects of each card prior to choosing did not improve performance, despite participants demonstrating that they understood the effects of each card. Evans (1972) disagreed with Wason's conclusion that this was a focal bias or verification bias. Instead, Evans argued that it was a matching bias (matching the surface terms used in the hypothesis). Evans found that when the problem was presented "If E then not V", participants chose the same cards as when asked to test "If E then V" (the E and V cards). However, Ormerod, Manktelow, and Jones (1993) found that by changing the instructions to E only if V enhanced performance. Platt and Griggs (1993) improved participants’ performance by instructing them to look for violations of the rule. Additionally, there have been many studies providing contradictory evidence as to whether or not a realistic context improves performance. (See Manktelow, 1999, for a review). 
Literature Review 22

Wason rule-discovery task studies. In the first study using the rule-discovery paradigm, Wason (1960) asked participants to formulate and test a hypothesis or rule to account for the data. In this paradigm participants are told that the series of numbers 2-4-6 follows a rule that the experimenter is thinking of. The participants are then asked to determine the rule by testing other series of three numbers, hereafter triads, by discovering whether they fit the rule or not. Participants are told to stop when they are sure what the rule is.

Wason (1960) found that participants typically chose the rule of even increasing numbers in sequence as their initial hypothesis. Participants usually then proceeded to test their hypothesis by asking about triads that fit their focal rule, following what has been called a "positive testing strategy” (Klayman \& Ha, 1987). Many studies have followed up this finding and have found that participants do not understand the value of a falsification strategy (Kareev \& Halberstadt, 1993). The primary way researchers have found to improve participant performance is to ask participants to consider or test more than one hypothesis simultaneously (Klayman \& На, 1989).

Classification studies. Classification studies are similar to rule discovery task studies, in that they require participants to create questions to test hypotheses. These studies ask participants to think up (presumably) diagnostic questions for classifying a person or object. For example, when participants were testing whether "Bob is an extrovert”, a typical question they used would be similar to, “Does Bob like to go to parties where he does not know anyone?” 
Literature Review 23

If the participants are choosing rationally, they will ask questions that they believe are diagnostic and whose diagnostic direction they know. Snyder and Swan (1978) found participants favored questions that they expected to be answered by a yes response if the focal hypothesis were true. Trope and Bassok (1982) disagreed, finding that people preferred evidence that would be diagnostic to evidence that would be highly probable if the hypothesis were true. Skov and Sherman (1986) found mixed evidence on this point. They found that people preferred diagnostic questions but, to a lesser extent, also preferred information that was likely given the hypothesis and therefore expected to be confirmatory.

Covariation and causation studies. Whether judging covariation or causation, participants have been shown to fail to consider all relevant data. Data have been presented either serially or in a two-by-two table. Smedslund (1963) found that even experts (nurses) fail to use all necessary information. He found the best predictor of participants’ judgment of covariation to be the present-present cell (both predictor and criterion present). Ward and Jenkins (1965), Shaklee and Tucker (1980), Shaklee and Mims (1982), and Arkes and Harkness (1983), using different methodologies, replicated the finding that the majority of participants' judgments are based on the present-present cell.

However, other studies have found evidence that participants use both predictor-present cells. Shaklee and Goldston (1989) and Shaklee and Hall (1983) both found that the most common strategy for participants was using only evidence when the possible cause was present. In contrast, using a serial presentation, Jenkins 
Literature Review 24

and Ward (1965) found that participants based their judgments of contingency on the present-present cell and the absent- absent cell.

Additional research has attempted to identify the variables relevant to participant's poor performance at judging covariation and causation. Evidence has been found that performance improves with age in youth (Shaklee and Goldston, 1989). Poor performance has been found to be due to limited memory (Shaklee and Mims, 1982), and sequential presentation (Ward \& Jenkins, 1965). The sequential method of presentation requires participants to store the evidence in working memory, which has been found to be limited to at most seven (see Simon, 1986 for a review) or as little as three (Broadbent, 1975) chunks of information. Thus this limitation of memory is probably the cause of poor performance in sequential presentation. Additionally, Jenkins and Ward (1965) found that their pre-training participants using examples of contingent and non-contingent sequences with feedback failed to improve in judgments of contingency.

Shaklee and Hall (1983) classified participants’ covariation strategies on the basis of a) participants’ verbal descriptions, b) participants' selections from a multiple choice list of verbal descriptions, and c) the patterns of participants' judgments. These three different methods of classifying covariation strategies produced only somewhat similar results (correlating between .45 and .58 with each other). Shaklee and Hall found it useful to interpret these correlations as reflecting two different types of participants, normative and non-normative. Participants who judged covariation normatively were more able to identify their strategies accurately. However, the 
Literature Review 25

participants who judged covariation non-normatively were less able to identify their strategies accurately.

Typically, these studies have presented subjects with all the information and therefore have no search phase. Shaklee and Goldston (1989), however, had participants choose which information to select and found that they did select information from all four cells, but the authors did not report the patterns of selection. Their analysis of participants' judgments was limited to the binary direction of contingency and was not analyzed in conjunction with the search phase data.

Dissonance theory studies. An entirely different paradigm with which to approach confirmation bias is based on Festinger's (1957) cognitive dissonance theory. Studies using this paradigm have examined the effects of a focal hypothesis on information search, presumably resulting in search that minimized cognitive dissonance (Jonas, Schulz-Hardt, Frey, \& Thelen, 2001). Cognitive dissonance theory predicts, among other things, that once a person accepts a position he or she will prefer to be exposed to information consistent with that belief and avoid information that is inconsistent with it, in order to avoid an experience of "cognitive dissonance” (Festinger, 1957).

Many studies have been conducted using this method (Frey, Schulz-Hardt, \& Stahlberg, 1996; Frey, 1986). Typically, participants are presented with real world problems and then their initial position is measured or manipulated. Next, participants are given an opportunity to expose themselves to additional information relevant to the correctness of their position. Participants choose additional information to read based 
on the titles that imply which side of the issue the information supports. Research under this paradigm has consistently found that people select and read more information that agrees with their existing beliefs (Jonas, Schulz-Hardt, Frey, \& Thelen, 2001). Recent research in this paradigm has found the same bias in information search prior to commitment, for alternatives that have emerged as promising (Russo, Medvec, \& Meloy, 1996; Luce, Bettman, \& Payne, 1997; Simon, Pham, Le, \& Holyoak, 2001)

As previously noted, this paper will use the term "predictor selection bias” to refer to this phenomenon of search biased in favor of variables expected to be confirmatory. (See page 4 for an explanation of this term). This bias has been replicated in many dissonance theory studies, across a variety of topics, methods and participants. Researchers have explored social stereotypes (Johnston, 1996), attitudes (Lundgren \& Prislin, 1998), negotiations (Pinkley, Griffith, \& Northcraft, 1995), selfimage (Frey, 1981), expert decision makers (bank managers) (Schulz-Hardt, Frey, Lüthgens, \& Moscovici, 2000), and motivated decision makers (Ditto \& Lopez, 1992).

\section{Consistent Findings Across Paradigms}

Many of the findings are similar across these seven paradigms. This suggests that the biases identified are not artifacts of the task or of the cover story, but reflect general cognitive processes. These similar findings include: failure to use base rate information, searching for less than maximally diagnostic information, searching for information associated with the focal hypothesis, integration of non-diagnostic information, and use of averaging integration strategies. 
Literature Review 27

Failure to use base rate information. Since, Kahneman and Tversky (1973) reported base rate neglect, it has been repeatedly replicated both in the Bayesian conditionals selection studies (Doherty, et al., 1979; Fischhoff \& Bar-Hillel, 1984) and in studies specifically examining the size and condition of the effect (see Bar-Hillel \& Fischhoff, 1981 for a review). The opposite effect, of under-revising for evidence has also been found in other circumstances and is called "conservatism” (Phillips and Edwards (1966), see Slovic and Lichtenstien, 1971 for a review).

Searching for less than maximally diagnostic information. There are two different ways that participants have sought less than maximally diagnostic information: seeking wholly non-diagnostic information and seeking minimally diagnostic information. The Bayesian conditionals selection studies have consistently found that people choose pseudodiagnostic information during the search phase (which is wholly non-diagnostic). Doherty, et al.’s (1979) finding of pseudodiagnosticity has been replicated in all studies in the paradigm (Doherty, et al., 1981; Beyth-Marom \& Fischhoff, 1983; Mynatt, et al., 1993). Similarly, most evidence points to the same bias in the Wason (1966) selection task. Wason (1996), and Wason and Johnson-Laird (1970) found that participants consistently chose the non-diagnostic evidence of Q (when testing If $\mathrm{P}$ then $\mathrm{Q}$ ). In the Wason rule discovery task, participants repeatedly used a positive testing strategy, which seeks minimally diagnostic information. In the classification studies, there is some support that people preferred questions that would be answered yes if the focal hypothesis were true to diagnostic questions (Snyder \& Swan, 1978; Skov \& Sherman 1986). These findings 
Literature Review 28

across paradigms indicate that participants do not grasp diagnosticity or its importance.

\section{Searching for information about or associated with the focal hypothesis.}

Searching for information that is about or associated with the focal hypothesis is the most consistent finding across paradigms. The Bayesian conditionals selection studies have repeatedly found this effect in the form of hypothesis focus bias (preference for information conditioned on the focal hypothesis) (Doherty, et al., 1979; Doherty, et al., 1981; Beyth-Marom \& Fischhoff, 1983; Mynatt et al., 1993). Similarly, Mynatt, et al. (1991) found the same preference for focal information using the Bayesian data sampling methodology. Research in the Wason selection task paradigm has often found a hypothesis focus bias to different degrees in different circumstances (preference for the P and Q cards when testing If P then Q) (Wason, 1966; Wason \& Johnson-Laird, 1970; Platt \& Griggs 1993; Ormerod, Manktelow, \& Jones 1993). Studies under the Wason (1960) rule discovery task have been consistent in their finding of a positive test strategy, testing new triads that are positive examples of their current hypothetical rule (Klayman \& Ha, 1987). In the covariation and causation literature, most studies do not have a search phase, however Shaklee and Goldston (1989) did. They found evidence of uneven searching, but did not report the direction of the bias.

Integration of non-diagnostic information. Ofir, (1988) using the Bayesian conditionals selection method found that participants often based their judgments on the hit rate $\left[p\left(\mathrm{D} \mid \mathrm{H}_{1}\right)\right]$ without using the complementary false alarm rate $\left[p\left(\mathrm{D} \mid \mathrm{H}_{2}\right)\right]$ 
Literature Review 29

(i.e., showed pseudodiagnostic integration). Kemmelmeier (2004), using the Bayesian data sampling method, found that the presence of non-diagnostic information had an impact on the outcome of integration (the dilution effect, discussed earlier). While, it is impossible to say whether pseudodiagnosticity was involved in the dilution effect, diagnosticity was clearly not properly weighed. The covariation and causation paradigm has consistently demonstrated participants’ reliance on insufficient data in making judgments, for example, integrating data most commonly from only a single row, column, or cell, instead of the entire table (Shaklee \& Goldstone 1989).

Use of averaging integration strategies. The Bayesian conditionals selection literature has found evidence that participants often use averaging strategies (Ofir, 1988; Fischhoff \& Bar-Hillel, 1984). Similarly, the Bayesian data sampling literature has also found the use of averaging strategies (Troutman \& Shanteau, 1977; Nisbett, et al., 1981; Kemmelmeier, 2004).

\section{Causes and Cure of Biases}

Attention. Participants' attention is the most widely researched cause of biases. In all seven paradigms every study examined attention. It was not manipulated as an independent variable but one hypothesis was assumed to be the focal hypothesis from the script. Doherty, et al., (1979) is the single exception to this, they measured the participants' belief about which hypothesis was more likely, and assumed that this hypothesis was now the focal hypothesis. The focusing of the participants' attention on one hypothesis is believed to be the primary cause of the search phase biases outlined in the paragraphs above (Searching for less than maximally diagnostic 
Literature Review 30

information and Searching information that is about or associated with the focal hypothesis).

Motivation. The dissonance theory paradigm is the only one to examine motivation. These studies manipulated the potential level of cognitive dissonance, which should in turn manipulate the participant's motivation (see Frey, 1986, for a review). These studies have consistently found that increased cognitive dissonance causes increased predictor selection bias.

Timing-of-feedback. Covariation and causation is the only paradigm to manipulate the timing (sequential vs. simultaneous) of feedback as an independent variable. Ward and Jenkins (1965) found that simultaneous feedback caused less frequent use of sub-optimal strategies.

Surprise. The possibility of a surprising result improving participants thinking has been explored in the Bayesian conditional selection paradigm. Ofir (1988) factorially manipulated the base rate, the hit rate and the false alarm rate. Ofir found that participants used the false alarm rate when the hit rate and base rate were seemingly inconsistent (which can be assumed to be surprising). Doherty, et al. (1981) manipulated surprise as an independent variable by providing half the participants an additional conditional probability that completed a matched pair of conditionals. The pair was constructed so that it would be inconsistent (and therefore surprising) with their assumed interpretation of the single conditional probability they already had. The participants who received the extra surprising conditional probability performed better (less pseudodiagnostic search) on later trials than those who did not receive it. 
Literature Review 31

\section{Problems with Current Research.}

Despite the areas of agreement outlined above, there remain three major problems with the current research: a) the common practice across paradigms of investigating only one phase of decision-making at a time; b) the lack of quantification of any effect of confirmation bias (as defined here); and c) a lack of research exploring predictor selection bias (with the exception of the Dissonance theory paradigm).

The first problem stems from the common practice of conducting studies that explore only one decision making phase at a time (search or integration). Research on “confirmation bias” (however defined) has commonly been conducted looking at only one of these phases in a given study. This practice prevents examination of any interaction between these phases, for example, asking whether information search phase strategy influences integration phase strategy. Additionally, this practice prevents following the effects of errors in the search phase to see whether they actually result in what is here referred to as confirmation bias in the final judgments. Table 6 lists the studies by paradigm and phases they examine. 
Table 6

Studies by Paradigm and Decision Phases Examined

\begin{tabular}{|c|c|c|}
\hline Paradigm & Search Studies & Integration Studies \\
\hline $\begin{array}{l}\text { Bayesian } \\
\text { conditionals }\end{array}$ & \multicolumn{2}{|c|}{$\begin{array}{l}\text { Doherty, et al. (1979); Doherty, et al. (1981); } \\
\text { Beyth-Marom \& Fischhoff (1983) }\end{array}$} \\
\hline $\begin{array}{l}\text { selection } \\
\text { Studies }\end{array}$ & Mynatt, et al. (1993) & $\begin{array}{l}\text { Fischhoff \& Bar-Hillel (1984); } \\
\text { Ofir (1988) }\end{array}$ \\
\hline $\begin{array}{l}\text { Bayesian } \\
\text { data } \\
\text { sampling } \\
\text { studies }\end{array}$ & Mynatt, et al. (1991) & $\begin{array}{l}\text { Phillips \& Edwards (1966); } \\
\text { Trautman \& Shanteau (1977); } \\
\text { Nisbett, et al. (1981); } \\
\text { Kemmelmeier (2004) }\end{array}$ \\
\hline $\begin{array}{l}\text { Wason } \\
\text { selection task } \\
\text { studies }\end{array}$ & $\begin{array}{l}\text { Wason (1966); Wason \& Johnson-Laird } \\
\text { (1970); Evans (1972); Ormerod, et al. } \\
\text { (1993); Platt \& Griggs (1993) }\end{array}$ & \\
\hline $\begin{array}{l}\text { Wason rule } \\
\text { discovery } \\
\text { studies }\end{array}$ & $\begin{array}{l}\text { Wason (1960); Klayman \& Ha (1987); } \\
\text { Klayman \& Ha (1989); Kareev \& } \\
\text { Halberstadt (1993) }\end{array}$ & \\
\hline $\begin{array}{l}\text { Classification } \\
\text { studies }\end{array}$ & $\begin{array}{l}\text { Trope \& Bassok (1982); Snyder \& Swan } \\
\text { (1978); Skov \& Sherman (1986) }\end{array}$ & \\
\hline \multirow{2}{*}{$\begin{array}{l}\text { Covariation } \\
\text { and causation } \\
\text { studies }\end{array}$} & \multicolumn{2}{|c|}{ Shaklee \& Goldston (1989) } \\
\hline & & $\begin{array}{l}\text { Smedslund (1963); Ward \& } \\
\text { Jenkins (1965); Jenkins \& Ward } \\
\text { (1965); Shaklee \& Tucker } \\
\text { (1980); Shaklee \& Mims (1982); } \\
\text { Arkes \& Harkness (1983); } \\
\text { Shaklee \& Hall (1983) }\end{array}$ \\
\hline $\begin{array}{l}\text { Dissonance } \\
\text { theory } \\
\text { studies }\end{array}$ & $\begin{array}{l}\text { Frey (1981); Frey (1986); Ditto \& Lopez } \\
\text { (1992); Pinkley, et al. (1995); Frey, et al. } \\
\text { (1996); Johnston (1996); Russo, et al. } \\
\text { (1996); Luce, et al. (1997); Lundgren \& } \\
\text { Prislin (1998); Schulz-Hardt, et al. (2000); } \\
\text { Simon, et al. (2001) }\end{array}$ & \\
\hline
\end{tabular}


Literature Review 33

In all seven paradigms, no study examines the effects of an individual's search strategy on his or her integration strategy or decision. In the seven paradigms, only four articles even reported data on both phases. In one of these, Shaklee and Goldston (1989), using the covariation and causation paradigm, reported data from both phases, but the integration data were limited to group-level data, which in addition were only binary. Group level data prevent the examination of any possible linkage between an individual's search strategy and his or her integration strategy and final judgment. The other three articles reporting data on both phases used the Bayesian conditionals selection paradigm. Doherty, et al. (1979) and Doherty, et al. (1981) were both primarily studies on the search phase. In Doherty, et al. (1979), integration data were collected only for the first preliminary step, which existed merely to demonstrate base rate neglect and to measure the focal hypothesis. Doherty, et al. (1981) replicated this first step of Doherty, et al. (1979) with minor changes. As with Shaklee and Goldston (1989), only group-level (binary) integration data were collected, again preventing the examination of any possible linkage between an individual's search strategy and his or her integration strategy and final judgment. Beyth-Marom \& Fischhoff (1983) examined both phases but only in separate experiments, once again preventing examination of this possible linkage.

The practice of studying search and integration phases separately implies the assumption that the search and integration phases are independent and can be appropriately treated as separate systems. Using the definition of a system proposed by Lendaris (1986), systems are composed of sub-units operating together to manifest 
Literature Review 34

emergent properties that are not apparent by examining the sub-units separately. In these terms, the entire Bayesian revision process may constitute a system. Phase interactions (for example search phase effects on integration strategy and search phase effects on probability revision) would constitute the emergent properties required by this definition of a system. The existence of phase interactions could be directly tested. Any evidence of the existence of phase interaction would attack the appropriateness of the view that search and integration are sub-units of a single belief-revision system. The finding that people are better at using information that is presented to them than at seeking it out suggests an interaction of this kind (Beyth-Marom \& Fischhoff, 1983).

This common practice of studying the two phases of decision making separately may contribute to the other common practice of using generic names that are not specific to a phase.

The second problem in the research literature is the lack of quantification of confirmation bias (as defined here). This lack of quantification could be caused by any combination of the following three factors. (1) The practice of studying each phase separately prevents the measurement of any confirmation bias caused by the search phase. (2) The imprecise use of any term (especially “confirmation bias”) impedes its quantification. Typically, the effects of biases are not actually measured in terms of change in the final judgment or decision. (3) The seven paradigms either can not or have not been structured to produce a precise correct normative answer on an interval scale against which participants’ responses could be compared. 
The third problem in the literature is a lack of research exploring predictorselection bias. As stated before, predictor-selection bias is a form of search phase bias, where participants choose to learn about predictors that they expect will have a confirmatory impact on their focal hypothesis. In the real world, people often have expectations as to the direction of diagnosticity of the predictors available. For example, a person gathering information about buying a car would likely know, prior to gathering the actual data, that safety information would more likely favor buying a Volvo while cost information would more likely favor buying a Ford. The only paradigm that has examined predictor-selection bias is the dissonance theory paradigm. Dissonance theory research has consistently found that participants favor information that agrees with beliefs to which they have committed (Jonas, SchulzHardt, Frey, \& Thelen, 2001). However, this paradigm has not quantified the confirmatory effect of this bias against a normatively correct standard, nor examined interactions with the integration phase. Additionally, this paradigm confounds two possible biases, a motivational one (from their commitment) and a cognitive one (from the participants' attention focus). 
Method 36

\section{The Present Study}

The present study is designed to overcome the three weaknesses in the existing research by a) investigating both phases of Bayesian revision, b) exploring predictor selection bias, and c) quantifying confirmation bias from sources in each phase.

The first decision in the design of this study is the choice of which of the seven paradigms to use. The Bayesian conditionals selection paradigm was chosen primarily in order to create a situation with a normative outcome on an interval scale, so that the combined effects of confirmation biases can be quantified. Of the seven paradigms, four do not yield a normative answer on an interval scale (Dissonance theory studies, Wason rule discovery task, Wason selection task, and classification studies). The dissonance theory studies typically do not even require integration, much less quantify the effects of new information. The Wason rule discovery task does not produce a normative outcome; the participant can never eliminate all the possible alternative rules with which the triads are consistent. While the Wason selection task produces a normative outcome; it is binary; the rule is disproved or not. The classification studies do not produce a normative outcome on an interval scale, because the diagnosticities of the questions are never explicitly quantified.

Two other paradigms, the covariation and causation studies and the Bayesian data sampling studies, do produce at least an interval-scale normative-outcome (the correlation coefficient and the probability of a sample having been drawn from a specific population, respectively). However, quantitative dependent measures suitable for comparison with these normative outcomes are problematic, because numeric 
values are not typically employed by average people in corresponding real world problems. (e.g., people do not normally quantify the effect on their beliefs upon hearing that the clouds have been seeded two days, and it rained both days, and not seeded two days and it rained once).

This leaves only the Bayesian conditionals selection paradigm as an appropriate paradigm for this research. An additional benefit of the Bayesian conditionals selection paradigm is that the problem of seeking conditional probability information is similar to that encountered in real world problems faced by ordinary people. The use of a real world situation instead of a purely logical framework may improve participants’ performance. Johnson-Laird, Legrenzi, and Legrenzi (1972) found a familiar real world context improved performance. However, later studies have found mixed support for this. See Manktelow (1999) for a review of this topic.

This paradigm lends itself naturally to the study of both phases of decisionmaking. Both phases have actually been studied using this paradigm, though usually in separate studies. This paradigm thus enables a researcher to follow Lendaris’ (1986) advice and study properties of the entire system as well as each sub-unit simultaneously.

Similarities to Doherty, et al. (1979). This study will use the same paradigm and cover story (with minor modifications) as Doherty, et al. (1979) and Doherty, et al. (1981), in order to facilitate direct comparisons with these experiments. The cover story used by Doherty, et al. (1979) told participants about an archeologist finding an urn between two islands and having to decide which island the urn came from. 
Differences from Doherty, et al. (1979). Although, the scripts in the present study describe a similar hypothetical situation (determining the island of origin of an urn), there are ten functional differences between these scripts and Doherty's, four of which have to do with four independent variables not manipulated by Doherty, et al. (1979).

First, in Doherty's scripts the focal hypothesis was measured for each subject, while in the present scripts, focal hypothesis will be manipulated as an independent variable "attention”. This variable has two levels: focused and balanced. Half of the participants will receive instructions that focus their attention on a single hypothesis and half will receive instructions that balance their attention. Doherty defined focal hypothesis to be the island that the participant judged to be more likely the origin of the urn after initial search and integration phases. In this study these initial search and integration phases are eliminated and replaced with an independent variable attention that has two levels, balanced and focused.

Second, these scripts manipulate motivation as an independent variable “motivation”. This variable has two levels: motivated and not motivated. Half of the participants will be provided a motivation for a preferred outcome and half not. This variable enables the study of the effects of motivation on search and integration strategies.

Third, these scripts manipulate timing as an independent variable "timing of feedback”. Half of the participants will seek information sequentially and half 
simultaneously. This enables an examination of the effects of search phase sequencing. (E.g., does early information affect subsequent search decisions?)

Fourth, these scripts manipulate the size of the conditional probabilities as an independent variable "size-of-conditionals". Half the participants will be in the high conditionals condition. When these participants select probabilities conditioned on the island favored by the hint, they will receive probabilities greater than $50 \%$. (See the next paragraph for an explanation of the hints.) The other half of the participants will be in the low conditionals condition. When these participants select probabilities conditioned on the island favored by the hint, they will receive probabilities less than $50 \%$. This manipulation is intended to surprise the subjects in the low condition and encourage them to think more carefully. This is similar to the effect found by Smith, Schoben and Rips (1974).

Fifth, these scripts add hints in the form of vague non-numeric information about the expected diagnostic direction of each of the conditional probabilities (i.e., "Characterizes the current clay pits of Shell Island”). These hints provide the basis for any predictor selection bias. Similar to those in the dissonance theory paradigm (Jonas, Schulz-Hardt, Frey, \& Thelen, 2001), this study’s hints accurately indicate the direction of diagnosticity.

Sixth, these scripts provide participants with a choice of 4 out of 16 conditional probabilities, where the 16 conditional probabilities form eight diagnostic pairs. Of these eight pairs, four have hints that favor the focal island and four have hints that favor the non-focal island. The 16 conditional probabilities can be classified by 2 
dimensions, the island they are conditioned on (the columns in Tables 7 and 8) and the island the relevant hint favors (the rows). Therefore, each cell has four of the 16 conditional probabilities in it, each of which can form a diagnostic pair with a conditional probability in the other cell in the same row.

Tables 7 and 8 illustrate the types of conditional probabilities that the participants may choose in Doherty, et al. (1979), and in the present study, respectively. (Note that these tables are not traditional covariation tables).

\section{Table 7}

The Types of Conditional Probabilities in Doherty, et al.'s (1979) Study

$\begin{array}{lcc}\text { Hints } & \begin{array}{c}\text { Conditional probabilities } \\ \text { about focal Island }\end{array} & \begin{array}{c}\text { Conditional probabilities } \\ \text { about non-focal island }\end{array} \\ \text { There are no hints to } & 6 & 6 \\ \text { distinguish the predictors } & \end{array}$


Table 8

The Types of Conditional Probabilities in the Present Study

\begin{tabular}{l|c|c}
\hline \multicolumn{3}{c}{$\begin{array}{c}\text { Conditional probabilities } \\
\text { about focal Island }\end{array}$} \\
\begin{tabular}{l|c} 
Conditional probabilities \\
about non-focal island
\end{tabular} \\
\hline Predictors with hints & 4 & 4 \\
favoring focal island & (Quadrant 1) & 4 \\
Predictors with hints & 4 & (Quadrant 3) \\
favoring non-focal island & (Quadrant 2) & \\
\hline
\end{tabular}

These changes (adding the hints and increasing the number of conditional probabilities to 16) enable participants to demonstrate any number of the following possible search phase patterns: a) cell bias, selecting all four conditional probabilities from the same category (which demonstrates a form of pseudodiagnosticity, hypothesis focus bias, and predictor selection bias); b) column bias, selecting all four conditional probabilities from a single column (which demonstrates a form of pseudodiagnosticity and hypothesis focus bias); c) pseudodiagnostic row bias, selecting all four conditional probabilities from a single row without forming any diagnostic pairs (which demonstrates a form of predictor selection bias and pseudodiagnosticity); d) diagnostic row bias, selecting two diagnostic pairs from a single row (which demonstrates a form of predictor selection bias despite being Bayesian), or e) unbiased Bayesian, selecting one diagnostic pair from each row. 
Method 42

Seventh, these scripts provide participants with conditional probabilities whose diagnosticity is held at a constant 2:1 ratio. This was done to ensure the same normatively correct outcome. (This is discussed further in the Methods Section).

Eighth, conditional probabilities and the base rate are presented as frequencies instead of probabilities. This was done following the advice of Gigerenzer \& Hoffrage (1995) that participants perform better with frequencies. This step was taken to make the problem easier for the participants, and thus to reduce random error. (Random responding has been a problem in other cognitive studies conducted by the author using the same participant pool).

Ninth, data will be collected and analyzed on both search and integration phase decisions, and the interactions between these phases will be examined.

Tenth, manipulations in the original studies of the base rate and the diagnostic direction of the conditional probabilities will be dropped. These changes were made to simplify the study and to ensure the same normatively correct outcome. The inclusion of both manipulations in the original study was to demonstrate base rate neglect, a phenomena which is now widely accepted.

\section{Hypotheses}

The hypotheses are divided into research hypotheses and exploratory hypotheses. The research hypotheses are significant extensions of prior research and will be evaluated by independent statistical tests. The exploratory hypotheses are not significant extension of prior research and/or can not be tested by independent statistical tests. This distinction is made between research and exploratory hypotheses 
to preserve the study wide alpha level. Only research hypotheses will be included in the study wide alpha level. Both research and exploratory hypotheses can be subdivided into experimental and non-experimental hypotheses, the difference being whether an independent variable is included in the hypothesis.

Expected effects of independent variables. The independent variables involved in the experimental hypotheses (both research and exploratory) are expected to produce biasing and unbiasing effects on arriving at posterior probabilities. These effects are expected to have consistent (biasing or unbiasing) effects across dependent variables regarding the participants’ search strategies, integration strategies, and posterior probabilities.

The independent variable attention is expected to create a focal hypothesis. Research across the seven paradigms has suggested that participants who have a focal hypothesis in mind are biased in both the search and integration phases.

The independent variable motivation is expected to create a motive to determine that one of the hypotheses is more likely. Many dissonance theory studies support the expectation that motivated participants will select more information expected to support their existing beliefs.

The independent variable size-of-conditionals presents conditionals that are either consistent with or violate participants' expectations. In the low condition, participants are expected to be surprised by probabilities below 50\%, when on the basis of the hint they were presumably expecting probabilities above 50\%. Such an effect would be consistent with Ofir’s (1988) finding that participants used the false 
alarm rate data $\left[p\left(\mathrm{D} \mid \mathrm{H}_{2}\right)\right]$ only if the base rate and the hit rate $\left[p\left(\mathrm{D} \mid \mathrm{H}_{1}\right)\right]$ were on different sides of .5 and therefore psychologically inconsistent. Doherty, et al. (1981) found a similar effect of surprise. Their study asked participants to choose two conditional probabilities from four (all four probabilities would form a diagnostic pairs). Participants who chose unmatched conditional probabilities were later given a third conditional probability which completed a diagnostic pair. Participants who received the third conditional probability changed their posterior probability estimate, presumably because the new information contradicted their assumed previous interpretation of unmatched conditional probabilities.

The independent variable timing of feedback has two levels: sequential and simultaneous. The sequential condition enables participants to alter their search strategy after having learning the results of earlier choices of conditional probabilities. It is expected that the combination of low conditionals and sequential timing of feedback will result in an unbiasing effect.

Thus the attention manipulation is expected to produce bias in the focused condition; the motivation manipulation is expected to produce bias in the motivated condition; and the interaction of timing of feedback and size of conditional in the combination of sequential and low conditions is expected to reduce bias. However, participants must be biased by attention and/or motivation in order to be unbiased by the combination of sequential feedback and low conditionals. Those participants in conditions expected to produce bias will be refereed to as biased participants (and the remaining participants will be referred to as unbiased participants). 
Method 45

Research hypotheses. The four research hypotheses are listed in Table 9 and discussed in the section below. In Table 9 each hypothesis is listed by number in the cells corresponding to the independent and dependent variables involved. 
Table 9

Research Hypotheses and their Associated Independent and Dependent Variables

\begin{tabular}{|c|c|c|c|c|c|}
\hline \multicolumn{6}{|c|}{ Research Hypotheses - Experimental } \\
\hline & $\begin{array}{l}\mathrm{IV}_{1} \\
\text { Attention }\end{array}$ & $\begin{array}{l}\mathrm{IV}_{2} \\
\text { Motivation }\end{array}$ & $\begin{array}{l}\text { IV }_{3} \text { Timing- } \\
\text { of-Feedback }\end{array}$ & $\begin{array}{l}\mathrm{IV}_{4} \text { Size-of- } \\
\text { Conditionals }\end{array}$ & $\begin{array}{l}\mathrm{IV}_{3} * \\
\mathrm{IV}_{4}\end{array}$ \\
\hline \multicolumn{6}{|l|}{ Search Process DVs } \\
\hline \multicolumn{6}{|l|}{$\mathrm{DV}_{1}$ Hypothesis Focus Bias } \\
\hline $\mathrm{DV}_{2}$ Predictor Selection Bias & R1 & R1 & & & R1 \\
\hline \multicolumn{6}{|l|}{$\mathrm{DV}_{3}$ Pairs Selected } \\
\hline \multicolumn{6}{|l|}{$\mathrm{DV}_{4}$ Search Strategy } \\
\hline \multicolumn{6}{|l|}{$\mathrm{DV}_{14}$ Search Surprise } \\
\hline \multicolumn{6}{|l|}{ Integration Process DVs } \\
\hline \multicolumn{6}{|l|}{$\begin{array}{l}\mathrm{DV}_{8} \text { Hypothesis-Focus } \\
\text { Integration Bias }\end{array}$} \\
\hline \multicolumn{6}{|l|}{$\begin{array}{l}\mathrm{DV}_{9} \text { Predictor-Focus } \\
\text { Integration Bias }\end{array}$} \\
\hline \multicolumn{6}{|l|}{$\begin{array}{l}\mathrm{DV}_{10} \text { Pairs Used in } \\
\text { Integration }\end{array}$} \\
\hline $\mathrm{DV}_{11}$ Integration Strategy & $\mathrm{R} 2$ & $\mathrm{R} 2$ & & & $\mathrm{R} 2$ \\
\hline \multicolumn{6}{|l|}{ Posterior Result DVs } \\
\hline $\mathrm{DV}_{5}$ Confirmation Bias & R3 & R3 & & & R3 \\
\hline \multicolumn{6}{|l|}{$\begin{array}{l}\mathrm{DV}_{6} \text { Search Confirmation } \\
\text { Bias }\end{array}$} \\
\hline \multicolumn{6}{|l|}{$\begin{array}{l}\mathrm{DV}_{12} \text { Integration } \\
\text { Confirmation Bias }\end{array}$} \\
\hline \multicolumn{6}{|l|}{$\mathrm{DV}_{13}$ Sum-of-Phase-Biases } \\
\hline \multicolumn{6}{|l|}{$\mathrm{DV}_{15}$ Intuitive Conditionals } \\
\hline \multicolumn{6}{|c|}{ Research Hypotheses - Non-Experimental } \\
\hline $\begin{array}{l}\text { R4 Use of biased search strat } \\
\text { strategies }\left(\mathrm{DV}_{11}\right) \text {. }\end{array}$ & ies $\left(\mathrm{DV}_{4}\right) \mathrm{w}$ & ll be positive & correlated witl & use of biased il & egration \\
\hline
\end{tabular}

Note: R1-R4 refer to the four research hypotheses and the IV DV combinations they examine. Many empty cells in this table will be examined by exploratory hypotheses. See Table 10 for exploratory hypotheses. 
The three experimental research hypotheses will be tested by planned comparisons within an ANOVA framework.

Predictor selection bias. Participants in conditions expected to produce bias will demonstrate predictor selection bias by selecting more conditional probabilities from minerals that are hinted to favor Shell Island than minerals hinted to favor Coral Island (the top row of Table 8). Predictor selection bias was found in a pilot study (Borthwick and Anderson, 2001) using a personnel selection cover story under the Bayesian conditionals selection paradigm. Additionally, predictor selection bias is analogous to the finding in the dissonance theory research that participants choose information that is expected to support their existing belief or leading choice. However, as previously noted, the dissonance literature has not clarified whether predictor selection bias was caused by motivation and/or attention focus, because these two variables were always confounded.

Research Hypothesis \#1. Biased Participants will select more conditional probabilities from minerals that are hinted to favor Shell Island than minerals hinted to favor Coral Island.

Biased Bayesian strategy in integration. The design of this study (specifically the use of hints in the Bayesian conditionals selection paradigm) creates the opportunity for participants who search optimally (selected one pair hinted to favor each hypothesis, as discussed under "Demonstration of the Optimal Search and Integration Strategies” in the methods section), to show bias in integration by using only one of the two pairs in integration (Biased Bayesians). (Biased Bayesians would 
use their pair from the top row but not their pair from the bottom row in Table 8). Biased participants are expected to show this bias despite searching evenly and using Bayes’ Theorem. This bias will result in a confirmatory effect in their posterior probability, by being more likely to use information that has been hinted to favor Shell Island in integration than information hinted to favor Coral Island.

This hypothesis extends the dissonance theory findings to the Bayesian Conditionals paradigm. Confirmation bias in integration is expected to show up despite unbiased search.

Research Hypothesis \#2. Biased Participants who searched optimally will be more likely in integration to use the selected pair favoring Shell than the pair favoring Coral.

Confirmation bias. Participants will demonstrate confirmation bias by producing a posterior probability that favors Shell Island. This extends prior findings in all seven paradigms that participants can be biased in the search and integration phases which will cause a bias in their resulting posterior probability (confirmation bias as defined in this study).

Research Hypothesis \#3. Biased Participants will produce posterior probabilities more in favor Shell Island than Coral Island.

Non-independence of phases. A major problem in the literature (noted above in Problems with Current Research) is that prior studies have examined the two phases of decision making separately. The two phases functioning separately is an underlying unstated and untested assumption. This assumption, on its face, appears false, since an 
individual's search strategy determines the information available to integrate and therefore must influence their integration. Additionally, past research has not compared participants’ judgments against a normative standard interval scale outcome. This study provides this standard and uses it to directly test the assumption that the phases are independent. This study assumes this assumption to be false and puts the assumption to a direct statistical test. If the assumption is true, an individual's choice of search phase strategy should be independent of their choice of integration phase strategy.

Research Hypothesis \#4. Participants who use search strategies that are hypothesis focused will be more likely to use integration strategies that are hypothesis focused.

Exploratory hypotheses. The exploratory hypotheses are listed in Table 10, and described below. The exploratory hypotheses can be sub-divided into experimental and non-experimental hypotheses, the difference being the inclusion of an independent variable in the hypothesis.

The hypotheses are listed by number, in the cells corresponding to the independent and dependent variables involved. Descriptions of each hypothesis follow. 
Table 10

Exploratory Hypotheses and their Associated Independent and Dependent Variables

\begin{tabular}{|c|c|c|c|c|c|}
\hline \multicolumn{6}{|c|}{ Exploratory Hypotheses - Experimental } \\
\hline & $\begin{array}{l}\mathrm{IV}_{1} \\
\text { Attention }\end{array}$ & $\begin{array}{l}\mathrm{IV}_{2} \\
\text { Motivation }\end{array}$ & $\begin{array}{l}\mathrm{IV}_{3} \text { Timing- } \\
\text { of Feedback }\end{array}$ & $\begin{array}{l}\mathrm{IV}_{4} \text { Size-of- } \\
\text { Conditionals }\end{array}$ & $\begin{array}{l}\mathrm{IV}_{3} * \\
\mathrm{IV}_{4}\end{array}$ \\
\hline \multicolumn{6}{|l|}{ Search Process DVs } \\
\hline $\mathrm{DV}_{1}$ Hypothesis Focus Bias & E1, E4 & E1, E4 & & & E1 \\
\hline $\mathrm{DV}_{2}$ Predictor Selection Bias & R1, E5 & R1, E5 & & & R1 \\
\hline $\mathrm{DV}_{3}$ Pairs Selected & E2 & E2 & & & E2 \\
\hline $\mathrm{DV}_{4}$ Search Strategy & E3 & E3 & & & E3 \\
\hline $\mathrm{DV}_{14}$ Search Surprise & E15, E16 & E15, E16 & & & $\begin{array}{l}\text { E15, } \\
\text { E16 }\end{array}$ \\
\hline \multicolumn{6}{|l|}{ Integration Process DVs } \\
\hline $\begin{array}{l}\mathrm{DV}_{8} \text { Hypothesis-Focus } \\
\text { Integration Bias }\end{array}$ & E6 & E6 & & & E6 \\
\hline $\begin{array}{l}\mathrm{DV}_{9} \text { Predictor-Focus } \\
\text { Integration Bias }\end{array}$ & E7 & E7 & & & E7 \\
\hline $\mathrm{DV}_{10}$ Pairs Used in Integration & E8 & E8 & & & E8 \\
\hline $\mathrm{DV}_{11}$ Integration Strategy & R2, E9 & R2, E9 & & & $\begin{array}{l}\text { R2, } \\
\text { E9 }\end{array}$ \\
\hline \multicolumn{6}{|l|}{ Posterior Result DVs } \\
\hline $\mathrm{DV}_{5}$ Confirmation Bias & R3 & R3 & & & R3 \\
\hline $\mathrm{DV}_{6}$ Search Confirmation Bias & E10 & E10 & & & E10 \\
\hline $\begin{array}{l}\mathrm{DV}_{12} \text { Integration Confirmation } \\
\text { Bias }\end{array}$ & E11 & E11 & & & E11 \\
\hline $\mathrm{DV}_{15}$ Intuitive Conditionals & & & & E14 & \\
\hline \multicolumn{6}{|c|}{ Exploratory Hypotheses - Non-Experimental } \\
\hline $\begin{array}{l}\text { R4 Correlation between bias se } \\
\text { E13a Bayesian integration pred } \\
\text { conditionals. } \\
\text { E13b Bayesian integration pred } \\
\text { E12 The actual posterior will n }\end{array}$ & $\begin{array}{l}\text { ch and intę } \\
\text { ts actual po } \\
\text { ts actual po } \\
\text { be equal to }\end{array}$ & $\begin{array}{l}\text { tion strategie } \\
\text { riors better w } \\
\text { ariors better tl } \\
\text { e Sum-of-Phy }\end{array}$ & $\begin{array}{l}\text { len incorporati } \\
\text { an regression. } \\
\text { e-Biases. }\end{array}$ & g intuitive & \\
\hline
\end{tabular}

Note: R1-R4 refer to research hypotheses and E1-E14 refer to exploratory hypotheses and the IV DV combinations the hypotheses examine. See Table 9 for research hypotheses. 
Method 51

\section{Exploratory search process hypotheses.}

Hypothesis focus bias. Research in five paradigms has shown that directed attention causes a focal search bias (Bayesian conditionals selection studies, Bayesian data sampling studies, Wason selection task studies, Wason rule discovery studies, and covariation and causation studies; see paragraph "Searching information that is about or associated with the focal hypothesis" above for details). (Hypothesis focus bias is the disproportionate selection from the left column of Table 8). The following hypothesis will replicate and extend these findings.

Exploratory Hypothesis \#1. Participants in biasing conditions will select more probabilities conditioned on Shell Island than will unbiased participants. Pseudodiagnosticity. The effects of focal search bias (preference for selecting probabilities conditioned on the focal island) should appear in two other search phase dependent variables, pairs selected and search strategy. These hypotheses are related to (and not independent of) Exploratory Hypothesis \#1, but use different dependent variables.

Participants' focal search bias creates a mismatch between the number of probabilities they chose conditioned on each island, and therefore interferes with their chance of selecting matched pairs. The preference for unmatched conditionals in biased participants has been found by research in the Bayesian conditionals paradigm (Doherty, et al., 1979; Doherty, et al., 1981; Mynatt, et al., 1993; Beyth-Marom \& Fischhoff, 1983). (Participants who are pseudodiagnostic will select conditional 
probabilities without selecting the matching probability conditioned on the other island. The two probabilities would be from the same row in Table 8).

Exploratory Hypothesis \#2. Participants in biasing conditions will select fewer diagnostic pairs than unbiased participants.

Focal search strategies. Similarly, the focal search bias should cause participants to use search strategies focused on the focal island. The difference between Exploratory Hypotheses 3 and 1 is the difference between participants' search strategy (which classifies only the consistent participants see Search Strategy in methods section), rather than the just the result of that strategy (their actual search selections).

Exploratory Hypothesis \#3. Participants in biasing conditions would use more hypothesis-focused search strategies and fewer control focused search strategies than unbiased participants Comparing the size of hypothesis focus and predictor selection biases. Both the attention manipulation and the motivation manipulation are expected to produce search phase effects on the number of probabilities; (a) conditioned on the focal island, and, (b) on dimensions hinted to favor the focal island. However, the attention manipulation is expected to have a stronger effect on the number of probabilities conditioned on the focal island (hypothesis focus bias, disproportionately selecting probabilities from the left column of Table 8), while the motivation manipulation is expected to have a stronger effect on the number of conditionals selected on 
dimensions hinted to favor the focal island (predictor selection bias, disproportionately selecting probabilities from the top row of Table 8).

Exploratory Hypothesis \#4. The hypothesis focus bias caused by the attention manipulation will be greater than the predictor selection bias caused by the attention manipulation.

Exploratory Hypothesis \#5. The predictor selection bias caused by the motivation manipulation will be greater than hypothesis focus bias caused by the motivation manipulation.

\section{Exploratory integration process hypotheses.}

Hypothesis-focus integration bias. Research across paradigms has shown that participant use non-diagnostic information in integration. (See above paragraph entitled Integration of non-diagnostic information.) There are three types of findings that show the use of non-diagnostic information. First, Beyth-Marom and Fischhoff's (1983) finding that half of their participants reported that $p(\mathrm{D} \mid \mathrm{H} 2)$ was irrelevant. Second, Ofir's (1988) finding that $p(\mathrm{D} \mid \mathrm{H} 2)$ was used only when the hit rate and base rate were psychologically inconsistent. Third, the majority of the covariation research finding that participants used the either only the present-present cell (Ward \& Jenkins, 1965; Shaklee \& Tucker, 1980; Shaklee \& Mims, 1982; Arkes \& Harkness, 1983) or both predictor present cells (Shaklee \& Goldston, 1989; Shaklee \& Hall, 1983) in making their covariation judgments.

It is hypothesized biased participants will select more probabilities conditioned on Shell Island (Hypotheses-Focus Bias, Exploratory Hypothesis \#1), and therefore 
they will have more available for them to use in integration. Therefore, participants will continue to be hypothesis-focused biased in the integration phase (HypothesesFocus Integration Bias).

Exploratory Hypothesis \#6. Participants in biasing conditions will report using more information conditioned on Shell Island than will unbiased participants. Predictor-focus integration bias. As stated in Research Hypothesis \#1, Dissonance Theory research has found that participants chose information expected to confirm their hypothesis (predictor selection bias). The finding is expected to continue into the integration phase (as predictor-focus integration bias, i.e., use of more information expected to confirm the focal hypothesis in their integration phase). Since participants will select more information expected to confirm, and therefore the information available to them during integration. (Predictor-focus integration bias would be the disproportionate use of probabilities from the left column of Table 8) Exploratory Hypothesis \#7. Participants in biasing conditions will use in integration more conditional probabilities hinted to favor Shell Island than will unbiased participants.

Pairs used in integration. A similar extension of the search phase applies to the integration of unmatched pairs. Since biased participants will have fewer pairs available to use from their search (Exploratory Hypothesis \#2) they will use fewer pairs in integration. This expected finding is extensively supported by research in the Bayesian conditionals paradigm (Doherty, et al., 1979; Doherty, et al., 1981; Mynatt, et al., 1993; Beyth-Marom \& Fischhoff, 1983). This hypothesis is classified as 
exploratory despite the fact that it has extensive support because it is not independent of Research Hypothesis \#1. As noted before, in order for subjects to have a hypothesis focus bias (Research Hypothesis \#1) they must also be pseudodiagnostic.

Exploratory Hypothesis \#8. Participants in biasing conditions will use in integration fewer diagnostic pairs than unbiased participants.

Use of biased integration strategies. As stated in Exploratory Hypothesis \#4, participants are expected to use search strategies that focus on the focal hypotheses. This pattern is expected to continue into the integration phase, where participants are expected to use integration strategies that focus on the focal hypothesis. This in part will be caused by the disproportionate amount of information available to them in the integration phase about the focal island. This effect is similar to (and not independent of) Exploratory Hypothesis \#6 (hypothesis-focus integration bias). However, this hypothesis uses a different dependent variable, (integration strategy), which uses information in addition to the conditionals participants stated they used (which is the sole basis of hypothesis-focus integration bias).

Exploratory Hypothesis \#9. Participants in biasing conditions will use more integration strategies that focus on the focal island than unbiased participants. Phase biases. As stated in Research Hypothesis \#4, participants are expected to produce confirmation bias in posterior probabilities. The confirmatory effect of participants' strategies (in terms of posterior probability) can be measured for both phases separately (by combining their actual strategies in one phase with optimal 
strategies in the other phase). It is expected that biased participants' will produce greater confirmatory bias in both their search strategies and their integration strategies. Exploratory Hypothesis \#10. Participants in biasing conditions will have a higher posterior probability produced from optimal integration and their actual search than unbiased participants.

Exploratory Hypothesis \#11. Participants in biasing conditions will have a higher posterior probability produced from optimal search and their actual integration than unbiased participants.

A major problem in the literature (noted above in Problems with Current Research) is that prior studies have examined the two phases of decision making separately. The two phases functioning separately is an underlying unstated and untested assumption. This study assumes this assumption to be false and puts the assumption to two direct statistical tests. First, if the assumption is true, an individual's choice of a search phase strategy should be independent of that individuals' choice of integration phase strategy. This was tested as research hypothesis \#4. Second, if the assumption were true, then the total confirmatory effect of the two phases should be equal to the sum of the individual phase confirmatory effects. However, Edwards (1968) found that people under adjust (conservatism) and therefore it is expected that participants will show less bias in their posteriors than the sum of their phase biases. 
Exploratory Hypothesis \#12. Participants’ actual confirmation bias will be less than the sum of their search confirmation bias and their integration confirmation bias.

Intuitive conditionals. Gigerenzer engaged in a debate with the research team of Kahneman and Tversky for over a decade over the extent and cause of cognitive biases (Kahneman \& Tversky, 1996, Gigerenzer, 1996). An ongoing issue between them was why participants perform better in some situations than others (including the use of frequencies rather than conditional probabilities, and in familiar situations as opposed to abstract ones) (Gigerenzer, 1991). One possible explanation for the conflicting findings is that participants make assumptions about the problems, and these unexamined assumptions affect their decisions (Cosmides \& Tooby, 1996, Nickerson, 1996).

One of the assumption participants could make about a problem is the size of the unknown (in this study unchosen) conditional probabilities (intuitive conditionals). Intuitive conditional probabilities could have an impact on participants' posterior probabilities. If intuitive conditional probabilities are used by participants, they could offer a partial explanation as to why participants sometimes perform better in situations they are familiar. In familiar situations participants might have more accurate intuitive conditionals or use them in different ways. Different models will be explored examining whether participants used intuitive conditionals in the integration phase, and whether participants' intuitive conditionals are affected by the size of the actual conditionals. 
Exploratory Hypothesis \#13a. Models using intuitive conditionals predict participants’ posterior probabilities better than models that exclude them. Exploratory Hypothesis \#13b. Models using intuitive conditionals predict participants’ posterior probabilities better when combined by Bayes’ Theorem than when combined by regression.

Exploratory Hypothesis \#14. The size of intuitive conditionals will be affected by the size of the actual conditionals.

Surprise hypotheses. These two hypotheses continue the logic of the previous hypotheses, that surprise will induce deeper and less biased thinking. For participants who are in the sequential condition, if are surprised by the feedback they have an opportunity to use a less biased search strategy. Therefore, participants who experience some form of surprise will exhibit less hypothesis focus bias and predictor selection bias.

Exploratory Hypothesis \#15. For participants searching sequentially and in the focused and/or motivated conditions, and if they reported being surprised it is expected that they would select less information conditioned on Shell Island than those not surprised.

Exploratory Hypothesis \#16. For participants searching sequentially and in the focused and/or motivated conditions, and if they reported being surprised it is expected that they would select less information hinted to favor Shell Island than those not surprised. 
Method 59

\section{Method}

\section{Study Overview}

Task overview. All participants were presented with the problem of estimating the probability as to which of two islands an urn was made. Participants were randomly assigned to one of two levels of each of four independent variables: attention, motivation, timing-of-feedback and size-of-conditionals. Attention had two levels: balanced, where the two islands were treated equally, and focused, where one island was mentioned more frequently and earlier. Motivation had two levels: motivated, where participants were provided a motivation reason for preferring Shell Island, and not motivated, where there was no motivational reason for preferring either island. Timing-of-feedback had two levels: sequential, where participants received the results of each of their conditional probabilities before selecting later conditional probabilities, and simultaneous, where participants received all four conditional probabilities at the same time. Size-of-conditionals had two levels: low, where the conditional probabilities all were below 50\%, and high, where some of the conditional probabilities were above $50 \%$.

Participants were given an even base rate (the two islands were initially equally likely) and they were offered their choice of four (out of sixteen) conditional probabilities to assist them in estimating the probability that the urn originated on each island. These sixteen conditional probabilities formed eight matching pairs (two conditional probabilities on the same dimension each conditioned on one of the two islands). Half of the eight matching pairs were hinted to favor each island as the 
source of the urn. Participants were asked to make an estimate of the probability the urn came from each island. Lastly participants were asked about the conditional probabilities they did not choose, about their integration methods, and demographic information.

Measures overview. Measurements primarily targeted participants’ decision making processes. They included examining their search choices, their integration method (both information used and combination strategy) and decision results. These measures can be sub-divided into those taken during the decision process (concurrent) and those taken after the fact (retrospective and demographic). The retrospective measures were taken after the fact, because if taken during the process they might have altered participant behavior. Those measures taken during the decision process (concurrent measures) can be further subdivided in search phase and integration phase measurements. (See Appendix A for a copy of the script).

Concurrent search phase measurements. Participants were asked to choose any four of 16 conditional probabilities. These 16 conditional probabilities formed eight matching pairs (two conditional probabilities on the same dimension each conditioned on one of the two islands). Of the eight dimensions four were hinted to favor each island as the source of the urn.

Concurrent integration phase measurements. Participants were asked to estimate the probability that the urn came from each island.

Retrospective measurements. Participants were also asked about which information they used in making their decision and the mathematical processes (if 
any) they used in combining the information. Participants were asked if they were surprised by any of the conditional probabilities they received in the search phase, and to estimate the value of the 12 conditional probabilities that they did not choose.

Demographic measurements. Demographic questions were asked at the end of the script.

\section{Participants}

Demographics. A total of 324 undergraduate psychology students enrolled at an urban university in the Portland metropolitan area were recruited to participate in this study. Participants were recruited primarily through their undergraduate classes, and most received extra credit for their participation. Participants approximated Portland's racial makeup with 3.4\% African American, 69.1\% Caucasian, 3.7\% Latino / Hispanic, 13.0\% Asian / Pacific Islander and 7.7\% other. A small number of participants refused to state their race (3.1\%). Participants approximated the gender balance of the psychology classes from which participants were recruited from with 68.8\% female. A small number of participants refused to state their gender (2.8\%). This sample was chosen largely for convenience, although it may roughly represent the general population of all people who attend college.

Missing data. All participants completed the study. However, some participants did not follow some of the instructions correctly, and were therefore dropped from all analyses that involved data collected at or after their deviation. On this basis, nine participants (out of the original 324) were dropped from all analyses, because they chose more than the four requested conditional probabilities. Similarly, 
one additional participant was dropped from the integration-phase analyses, because that participant did not provide a posterior probability. Similarly, 72 participants were dropped from the intuitive conditionals analyses, because they did not provide all 12 requested intuitive conditionals.

\section{Experimental Design}

Structural design. This study employed a $2 * 2 * 2 * 2$ (attention, motivation, timing of feedback, size-of-conditionals) factorial between-subjects design. The between subjects design was used because order effects would have been a severe problem using a within-subject design.

\section{Design features used to ensure a single optimal search and integration}

strategy. This study had four design features to create a situation where there is both a single optimal search strategy and, assuming Bayesian revision, a single optimal posterior probability for all 16 conditions. There are four such design features. (1) The base rate was in all cases even (.50). (2) All participants were instructed to choose four conditional probabilities. (3) An equal number of dimensions were hinted to favor each island. (4) All matched pairs had the same diagnostic ratios.

Given (1), (2), and (3), the optimal search strategy is to select one diagnostic pair hinted to favor each island. A demonstration of the optimal of the search strategy is given in the section entitled "Demonstration of the Optimal Search and Integration Strategies”.

Theoretical definitions of independent variables. There are four independent variables in this study (attention, motivation, timing-of-feedback and size-of- 
conditionals). Additionally, different conditions of these four independent variables are expected to produce various confirmatory biases in participants while other conditions are expected to have no biasing impact.

The first independent variable attention is defined as whether the situation focuses participants' attention on one hypothesis or balances participants' attention between the two hypotheses.

The second independent variable motivation is defined as whether the situation provides the participants with a reason to prefer one hypothesis over the other or provides the participants no reason to prefer either hypothesis.

The third independent variable timing-of-feedback is defined as whether participants receive the results of their search choices before making the remaining choices or not.

The fourth independent variable size-of-conditionals is defined as whether the probabilities conditioned on the hypothesis that is hinted to be favored for that dimension are above or below 50\%.

Biasing conditions are conditions where the situations encourage participants to be biased and additionally are not conditions that both surprise the participants and provide them an opportunity to change their search strategies after such surprise. All other conditions are unbiased. (See section entitled "Biased and Unbiased Conditions” for a discussion). 
Theoretical definitions of dependent variables. Hypothesis-Focus bias (dependent variable 1) is the tendency to prefer evidence about the focal hypothesis to evidence about the alternative hypothesis.

Predictor-Selection bias (dependent variable 2) is the tendency to prefer evidence expected to support the focal hypothesis to evidence expected to support the alternative hypothesis.

Pairs selected (dependent variable 3) is the frequency of selecting matching pairs of conditional probabilities as opposed to unmatched conditional probabilities (pseudodiagnostic search).

Search strategy (dependent variable 4) is a classification of participants' search strategies. Search strategies are categorized by their use of matched pairs and from which of the four quadrants a participant selects conditional probabilities.

Confirmation bias (dependent variable 5) is the size and direction of participants’ probability estimates’ deviation from the normatively correct probability estimate.

Search confirmation bias (dependent variable 6) is the size of the confirmation bias (dependent variable 5) caused by the participants’ search.

Biased usage of hints (dependent variable 7) is the preference to use hints that favor the focal hypothesis as opposed to hints that favor the alternative hypothesis.

Hypothesis-Focus integration bias (dependent variable 8) is the preference to use in the integration phase evidence about the focal hypothesis to evidence about the alternative hypothesis. 
Predictor-Focus integration bias (dependent variable 9) is the preference to use in the integration phase evidence expected to support the focal hypothesis to evidence expected to support the alternative hypothesis.

Pairs used in integration (dependent variable 10) is the tendency to use matched pairs of conditionals in integration (diagnostic integration) as opposed to unmatched pairs (pseudodiagnostic integration).

Integration strategy (dependent variable 11) is a classification of participants’ integration strategies. These strategies are categorized by their use of mathematical operations and from which of the four quadrants conditional probabilities are used.

Integration confirmation bias (dependent variable 12) is the size of the confirmation bias (dependent variable 5) caused by the participants’ integration method.

Sum-of-Phase-Biases (dependent variable 13) is the sum of search confirmation bias (dependent variable 6) and integration confirmation bias (dependent variable 12). This reflects the confirmation bias caused by the two phases if they did not have any interactive effects.

Search surprise (dependent variable 14) is the degree that participants are surprised by the size of the conditional probabilities.

Intuitive conditionals (dependent variable 15) are participants' estimates of the unchosen conditional probabilities. 
Bayesian intuitive posterior (dependent variable 15a) is the posterior probability estimate a participant would have produced if they used all intuitive and selected conditional probabilities and combined them using Bayesian integration.

Bayesian matching posterior (dependent variable 15b) is the posterior probability estimate a participant would have produced if they used all selected conditional probabilities and any intuitive conditionals necessary to make matching diagnostic pairs and combined them using Bayesian integration.

Regression intuitive posterior (dependent variable 15c) is the posterior probability estimate a participant would have produced if they used all intuitive and selected conditional probabilities and combined them using the regression weights from a regression equation of all participants posteriors and conditional probabilities (both intuitive and selected).

Cell sizes. The numbers of participants, both original and final, in the various experimental conditions are provided in Table 11. 
Table 11

Participants Completing each Phase in each Condition.

\begin{tabular}{|c|c|c|c|c|}
\hline \multirow[t]{2}{*}{ Condition } & \multirow[t]{2}{*}{ Total } & \multirow{2}{*}{$\begin{array}{l}\text { Search } \\
\text { Phase }\end{array}$} & \multirow{2}{*}{$\begin{array}{l}\text { Integration } \\
\text { Phase }\end{array}$} & \multirow{2}{*}{$\begin{array}{l}\text { Retrospective } \\
\text { Measures }\end{array}$} \\
\hline & & & & \\
\hline 1 Balanced, Not Motivated, Sequential, Low & 21 & 20 & 20 & 13 \\
\hline 2 Balanced, Not Motivated, Sequential, High & 20 & 20 & 20 & 17 \\
\hline 3 Balanced, Not Motivated, Simultaneous, Low & 20 & 19 & 19 & 14 \\
\hline 4 Balanced, Not Motivated, Simultaneous, High & 20 & 18 & 18 & 15 \\
\hline 5 Balanced, Motivated, Sequential, Low & 21 & 21 & 21 & 18 \\
\hline 6 Balanced, Motivated, Sequential, High & 20 & 20 & 20 & 18 \\
\hline 7 Balanced, Motivated, Simultaneous, Low & 21 & 20 & 20 & 18 \\
\hline 8 Balanced, Motivated, Simultaneous, High & 21 & 20 & 20 & 12 \\
\hline 9 Focused, Not Motivated, Sequential, Low & 21 & 21 & 21 & 17 \\
\hline 10 Focused, Not Motivated, Sequential, High & 20 & 20 & 20 & 16 \\
\hline 11 Focused, Not Motivated, Simultaneous, Low & 21 & 21 & 21 & 16 \\
\hline 12 Focused, Not Motivated, Simultaneous, High & 20 & 20 & 20 & 12 \\
\hline 13 Focused, Motivated, Sequential, Low & 20 & 19 & 19 & 12 \\
\hline 14 Focused, Motivated, Sequential, High & 19 & 19 & 19 & 14 \\
\hline 15 Focused, Motivated, Simultaneous, Low & 19 & 18 & 18 & 15 \\
\hline 16 Focused, Motivated, Simultaneous, High & 20 & 19 & 18 & 16 \\
\hline Total & 324 & 315 & 314 & 243 \\
\hline Total Sequential Conditions Only & 162 & 160 & 160 & 125 \\
\hline
\end{tabular}


Method 68

\section{Operational definitions of independent variables}

All four independent variables had two levels and were manipulated in a factorial design.

IV $_{1}$ Attention operational definition. The attention manipulation has two levels, balanced and focused. The two levels were differentiated by three sections on the first page of the script (See appendix A for copy of the script). The focus of participants' attention was manipulated using four techniques, over the three parts of the attention manipulation, to restrict participants' attention in the treatment condition. First, attention was restricted by delaying the first mention of Coral Island (the alternative not mentioned until the third part). The delay in informing the subjects of the existence of and the evidence favoring Coral Island made use of the primacy effect (Jahnke, 1965) to strengthen the impact of the pro-Shell Island information. Second, attention was restricted by delaying the mention of a uniform base rate. Third, attention was restricted by reducing the frequency with which Coral Island was mentioned. Fourth, attention was restricted by altering the form in which the information was presented, prose or table (In the balanced condition a table is presented in part two, in the focal condition all the information is provided in prose in parts two and three). Because Ward and Jenkins (1965) had found that information that is presented in a table encourages participants to examine data relevant to both hypotheses, it is believed that use of a table will incline subjects towards diagnostic, instead of pseudodiagnostic, strategies and thus against the experimenter's hypotheses. 
In the focused condition the first two script sections were (the third section was omitted for the focused condition):

Imagine you are an archeologist specializing in South Sea cultures. Your research has led you to believe that the legendary Urn of Zor actually existed and was created on either Shell Island or nearby Coral Island, two islands that share a unique culture. You have found this Urn of Zor, resting on the ocean floor between Shell and Coral Islands.

Of the 10 urns that have previously been found at this spot on the ocean floor, 5 have been determined to have been made on Shell Island and 5 on Coral Island. Since there are no other islands with the same culture as Shell and Coral, no other island of origin is possible.

You need to make a determination as to the island on which the urn was more probably made. Your first step is to analyze the mineral content of the Urn of Zor. You find that the Urn of Zor has high content of the 8 minerals listed in the table below. Your second step is to determine the content of these minerals in the clay pits now in use on the two islands. The table below displays the results of the mineral content of the Urn of Zor and that of the current clay pits on the two islands: 


\begin{tabular}{|l|l|}
\hline $\begin{array}{l}\text { Mineral found to be in } \\
\text { abundance in the Urn of }\end{array}$ & $\begin{array}{l}\text { Island whose current clay pits are } \\
\text { characterized by an abundance of this } \\
\text { mineral }\end{array}$ \\
\hline Aluminum & Shell Island \\
\hline Calcium & Shell Island \\
\hline Chromium & Shell Island \\
\hline Copper & Shell Island \\
\hline Iron & Coral Island \\
\hline Magnesium & Coral Island \\
\hline Nickel & Coral Island \\
\hline Zinc & Coral Island \\
\hline
\end{tabular}

Unfortunately, the islanders have migrated around the islands over the years and have changed the clay pits they use. While the mineral content of the current clay pits is probably similar to the mineral content of those clay pits used in ancient times to make the Urn of Zor, it is certainly not identical. Therefore you will need an additional step in your analysis. At this point what is the probability the urn came from:

Shell Island ?

Coral Island ? 
In the focused condition the three script sections were: Imagine you are an archeologist specializing in South Sea cultures. Your research has led you to believe that the legendary Urn of Zor actually existed. While conducting underwater excavations, from your research facility on Shell Island, you have found this Urn of Zor, resting on the ocean floor.

When you return to your research facility on Shell Island, your first thought is to analyze the mineral content of the Urn of Zor. The results indicate that the Urn of Zor has a high content of eight minerals (Aluminum, Calcium, Chromium, Copper, Iron, Magnesium, Nickel and Zinc). Four (Aluminum, Calcium, Chromium and Copper) of these high content minerals are characteristic of the clay pits in current use on Shell Island. When you tell your research team the results, one of your co-workers states that some of these minerals remind him of the current clay pit on the side of Shell Island he has been working on. A second co-worker states that some of these minerals remind her of the current clay pit on another side of Shell Island on which she has been working. This sets off a furious debate among your research team as to the side of Shell Island on which the Urn was more probably made. Unfortunately, the evidence is not conclusive because, the islanders have migrated around the islands over the years and have changed the clay pits they use. While the mineral content of the current clay pits is probably similar to the mineral content of those clay pits used in ancient times to make the Urn of Zor, 
it is certainly not identical. Therefore you will need an additional step in your analysis.

At this point what is the probability the Urn came from anywhere on Shell Island ?

You announce the finding of the Urn of Zor and your intention to give it to the Shell Island National Museum, which you believe to be the most appropriate representative of the rightful owners. Coral Island, which has the same culture as Shell Island, quickly requests that you investigate the possibility that the Urn might have been made on their island, and requests that you not give the Urn to the Shell Island National Museum until you confirm that the Urn came from Shell Island.

Coral Island argues that of the 10 urns that have previously been found at the spot on the ocean floor where the Urn of Zor was found, 5 have been determined to have been made on Shell Island and 5 on Coral Island. Since there are no other islands with the same culture as Shell and Coral, no other island of origin is possible.

Additionally, they argue that despite the fact that four of the minerals (Aluminum, Calcium, Chromium and Copper) characterize the current clay pits of Shell Island, the other four minerals (Iron, Magnesium, Nickel and Zinc) characterize the current clay pits of Coral Island. 
$I_{2}$ Motivation operational definition. The motivation manipulation has two levels, not motivated and motivated. The two levels were differentiated by two sections of the script on the first page. The primary different was the inclusion in the motivated condition of a mention of a career incentive to determine that the urn came from Shell Island. In the not motivated condition the script section was (the second section was omitted for the motivated condition):

The Urn of Zor is believed to have been created soon after the first settlements on the islands. Finding the Urn provides new information about the early colonization of the islands and will trigger new interest and research opportunities for you and your fellow South Sea archeologists and greatly enhance your prestige. This is especially important to you, since you are coming up for promotion and tenure next year.

In the motivated condition the two script sections were:

If it could be established that this urn had come from Shell Island, this would dispute the legend that Shell Island was the last island in the South Sea area to be settled. This would provide abundant research opportunities for you and your fellow South Sea archeologists and greatly enhance your prestige. This is especially important to you, since you are coming up for promotion and tenure next year. 
Would it be good for your career if the probability were higher or lower, that the Urn came from Shell Island?

IV $_{3}$ Timing-of-feedback operational definition. The timing-of-feedback manipulation has two levels, sequential and simultaneous. The two levels were differentiated by the instructions at the top of the third page of the script. In both conditions the script read: "Choose 4 of the following 16 tests to perform, as the most efficient way to determine the origin of the Urn of Zor, following the instructions below.” In the simultaneous condition, participants were instructed to choose their conditional probabilities all at once before receiving feedback. In the sequential condition, participants were instructed to choose their conditional probabilities one at a time with feedback after each selection, thus enabling them to choose their later selections on the basis of the results of earlier choices.

Having participants in the Simultaneous Condition determine the order of importance of their selections, despite this not being essential to their task, was intended to balance the cognitive load between the two conditions of timing of feedback. This additionally provided a sense of relative importance of the four conditional probabilities. Having a measure of order in both conditions also made possible additional comparisons between conditions (especially for effects of $\mathrm{IV}_{3}$ timing-of-feedback). This added task (numbering the choices) should increase cognitive load, which might, in turn, encourage the use of simple heuristics. Biggs, Bedard, Gaber and Linsmeier (1985) found an effect of cognitive load, while Payne (1976) did not. Such an effect, if it exists, would work in the opposite direction of the 
possible effect from the use of tables, which is biasing towards proper use of diagnostic information.

In the sequential condition the script was:

Put the number 1 next to the first test you would like to perform, and then remove the corresponding sticker to reveal the test result.

Next choose your second test, put the number 2 next to that test and then remove the corresponding sticker to reveal the test result.

Next choose your third test, put the number 3 next to that test and then remove the corresponding sticker to reveal the test result.

Finally, choose your fourth test, put the number 4 next to that test and then remove the corresponding sticker to reveal the test result.

In the simultaneous condition the script was:

Put a number (1 to 4 ) next to the four tests you would like to perform in the order of importance to you ( 1 being top priority, 4 being lowest). Then remove the four corresponding stickers to reveal the four test results.

$\mathrm{IV}_{4}$ Size-of-conditionals operational definition. The size-of-conditionals manipulation has two levels, low and high. The two levels were differentiated by the size of the conditional probabilities the participants learn on the third page. In the low condition the conditional probabilities were $10 \%, 20 \%, 30 \%$, and $40 \%$ for the probabilities conditioned on the island favored by the hint, and the corresponding conditional probabilities were 5\%, 10\%, 15\%, and 20\% for the probabilities conditioned on the island not favored by the hint. In the high condition the conditional 
probabilities were $60 \%, 70 \%, 80 \%$, and $90 \%$ for the probabilities conditioned on the island favored by the hint, and the corresponding conditional probabilities were $30 \%$, $35 \%, 40 \%$, and $45 \%$ for the probabilities conditioned on the island not favored by the hint.

These conditional probabilities $\left[p\left(\mathrm{D} \mid \mathrm{H}_{1}\right)\right.$ and $\left.p\left(\mathrm{D} \mid \mathrm{H}_{2}\right)\right]$ were designed with three features. First, they were diagnostically consistent with the hints, to preserve the participants' faith in the value of the hints (especially for those in the Sequential Condition). Second, all conditional probability pairs had a diagnosticity ratio of 2:1 (discussed further below in Demonstration of the Optimal Search and Integration Strategies). For Bayesian participants, the 2:1 ratio would eliminate error variance due to random choice among the four diagnostic pairs hinted to favor the same island. Third, the sizes of the conditional probabilities within a category presented to any one subject were all either above or below 50\%. For participants who use a non-Bayesian integration strategy, this third feature would reduce random error due to selection of conditional probabilities within a quadrant.

The variation that remained among the conditional probabilities in each quadrant provided two benefits. First, it helped hide the fact that all the diagnostic pairs had the same diagnostic ratio. Because participants could pick only four conditional probabilities, they were not able to discover that the ratios were the same until they had picked their fourth and last conditional probability. Second, it limited the ability of non-Bayesians to accurately predict or make any assumption about the size of the non-chosen conditional probabilities (that is to use “intuitive conditionals”). 
Biasing conditions operational definition. The four independent variables (attention, motivation, timing-of-feedback and size-of-conditionals) were expected in different combinations to produce both biasing and unbiasing effects (see Expected Effects of Independent Variables for a discussion and Table 22 for a list of research hypotheses). The focal condition (attention IV) and the motivated condition (motivation IV) were each expected to cause participants to be biased in search and integration. The combination of sequential (timing-of-feedback IV) and low conditionals (size-of-conditionals IV) was expected to undo both of these biasing effects of both the focal and motivated conditions. Therefore, experimental conditions were considered biasing if they involved one or more of the biasing conditions (focused or motivated) and did not involve the unbiasing combination (sequential feedback and low conditionals). The biasing effects were expected to be consistent (biasing or unbiasing) across dependent variables regarding the participants’ search strategies, integration strategies, and posterior probabilities. This classification of the 16 conditions into biasing or non-biasing was treated as a dichotomous independent variable.

\section{Operational Definitions of Direct Dependent Variables}

Operational definitions of direct dependent search phase variables. Three search phase dependent variables $\left(\mathrm{DV}_{1}-\mathrm{DV}_{3}\right)$ were directly based on the participants' four choices of conditional probabilities selected from the sixteen available conditional probabilities. Each of the sixteen available conditionals was classified on the basis of whether it was conditioned on Shell Island or Coral Island and whether the 
mineral was hinted to be more abundant on Shell Island or on Coral Island. These two dimensions produce four quadrants, each of which has four conditional probabilities in it. The four conditionals in each quadrant differ in the mineral they provide frequency information about. The four quadrants are labeled as 1 through 4 as in Table 12 .

Table 12

Description of the Four Quadrants of Conditional Probabilities

Direction of $\quad p$ Conditioned on Shell Island $\quad p$ Conditioned on Coral Island
Hint

\begin{tabular}{lcc}
\hline Hint Favors & Quadrant 1 (4 conditional & Quadrant 3 (4 conditional \\
Shell Island & probabilities) & probabilities) \\
Hint Favors & Quadrant 2 (4 conditional & Quadrant 4 (4 conditional \\
Coral Island & probabilities) & probabilities) \\
\hline
\end{tabular}

Participants’ search phase choices of these four types of conditional probabilities were entered into two classification systems. The first system is limited to the four conditionals selected, while the second system includes all 16 available conditionals from which the four choices were made. In the first system (Quadrant System), each of the four choices was classified as to which of the four quadrants it fell into and whether or not it completed a diagnostic pair. In the second system 
Method 79

(Conditional System), what was recorded, separately for each of the possible 16 choices, was whether that conditional was selected or, if not selected, what frequency the participant estimated for that unchosen conditional probability. (So as not to introduce any bias into the primary task of producing a posterior estimate, this estimated frequency was obtained only after the completion of the posterior estimate.) Search phase dependent variables were derived either directly or indirectly from the variables of the Quadrant and Conditional systems. Many of the dependent variables that were created indirectly are based on frequency totals. From the Quadrant System data, Quadrant totals were summed over the four choices (e.g. if a participant choose 2 matching pairs that were hinted to favor Shell island, then these choices were described as 2 choices in quadrant 1 and 2 choices in quadrant 3, see Table 12). All coding except for integration search strategies was done by if / then logic commands in excel.

$D V_{1}$ : Hypothesis focus bias. Hypothesis focus bias was created by summing the total number of conditional probabilities selected from quadrants 1 and 2 . It has a possible range from 4, in which every choice is conditioned on the focal island (Shell), to 0 , in which every choice is conditioned on the non-focal island (Coral). Possible values are 4, 3, 2, 1, and 0 (See Table 13 for Coding). 
Method 80

Table 13

Coding for Hypothesis Focus Bias

\begin{tabular}{l|cc} 
Direction of Hint & $p$ Conditioned on & $p$ Conditioned on \\
& Shell Island & Coral Island \\
\hline Hint Favors Shell Island & +1 & 0 \\
Hint Favors Coral Island & +1 & 0
\end{tabular}

$\mathrm{DV}_{2}$ : Predictor selection bias. Predictor selection bias was created by summing the total number of conditional probabilities selected from quadrants 1 and 3 . It has a possible range from 4, in which every choice was hinted to favor the focal island (Shell Island), to 0, in which every choice was hinted to favor the non-focal island (Coral Island). Possible values are 4, 3, 2, 1, and 0 (See Table 14 for Coding).

Table 14

Coding for Predictor Selection Bias.

\begin{tabular}{lcc}
\hline Direction of Hint & $p$ Conditioned & $p$ Conditioned \\
& on Shell Island & on Coral Island \\
& & \\
\hline Hint Favors Shell Island & +1 & +1 \\
Hint Favors Coral Island & 0 & 0 \\
\hline
\end{tabular}


$D V_{3}$ : Pairs selected. This variable was the total number of diagnostic pairs selected by each participant (A diagnostic pair is a pair of conditional probabilities about the same mineral, one conditioned on each island). This total was calculated from the pair-completed data in the Quadrant System data. The total number of pairs was summed (using the MS Excel if / then command), creating a range from 0 to 2 pairs (resulting from the four conditional probability choices). Values include 0 (completely non-diagnostic search), 1, and 2 (completely diagnostic search). (For reference purpose, random selection would result in an average of .4 pairs).

\section{Operational definitions of direct dependent integration phase variables.}

$D V_{5}$ : Confirmation bias. Confirmation bias was derived from participants responses to the three questions at the bottom of the third page designed to elicit their revised probability that the Urn came from Shell Island:

Which Island do you now believe the Urn of Zor is more likely to have come from?

What do you now believe is the probability that the Urn of Zor came from that island?

What do you believe is the probability that the Urn of Zor came from the other island?”

Confirmation bias was calculated by subtracting the normative revised probability estimate that the Urn had been created on Shell Island (.50 in this scenario) from the participants' revised probability estimates. Because the subtraction of .50 is a linear transformation, it does not alter the statistical properties of the variable. 
However, it does make it easier to interpret and to compare to the other dependent variables that measure bias. For example, if a participant thought that the probability that the Urn came from Shell Island was $=.9$, then his or her confirmation bias score would be .4 (the revised estimate of .9 - the normative probability of .5).

The resulting variable ranges from +.50 , meaning the participant's revised estimate favored Shell Island .50 more than normative (maximum confirmatory bias), to -.50 , meaning the participant's revised estimate favored Coral Island .50 more than normative (maximum disconfirmatory bias).

The participant's answer to the first question determined which island the second and third questions were referring too. The participant's answer to the question referring to Shell Island was accepted as the participants’ posterior estimate of the probability that the Urn was made on Shell Island. The revised probability data were obtained in three parts in order to be as non-biasing as possible. This question format did however make the assumption that the participants' revised estimates of the probability of the Urn originating on Shell Island and originating on Coral Island add to 1 . While probability judgments involving many categories have been commonly shown to violate additivity, judgments of two categories have been commonly found to be additive (Wright \& Whalles, 1983). The third question about the probability of the less likely island provides a check on additivity. As long as a participant's answers to the two questions add up to $100 \%$ there has been no violation of additivity. If the departures from additivity seem to warrant it, then Departures from Additivity can be examined as a moderator variable. 
When subjects who found probability estimation difficult asked for assistance, the experimenter used the bi-section method to assist subjects in determining this probability (Torgerson, 1958; Pfanzagl, 1968; Seaver, von Winterfeldt, \& Edwards, 1978) This method asks the participant a series of questions all of the same form, do you think the probability is above or below $\mathrm{X}$, where $\mathrm{X}$ is chosen to be the point halfway between the ends of the range of possible values. (Thus, the first question will be is the probability above or below .50, if they answer it is above, the second question will be is the probability above or below .75 etc.).

Information usage dependent variables. The fourth page asked participants what information they used to make their revised probability estimates. The information used need not correspond to the information selected, because participants were free a) to ignore information they selected and b) to use information (the hints and base rate) they did not select but were provided. They were asked in closed ended format: "What information did you use in making this estimate of the probability of the island of origin? Put a check on the right next to all the pieces of information below that you used.”

Participants were then presented with a list of possibilities that included the base rate, the hints, and the particular conditional probabilities that they had selected. Additionally, a blank space was provided, in case a participant used any additional information not presented in closed-ended format. The responses to the closed-ended questions were used directly to create four dependent variables $\left(\mathrm{DV}_{7}-\mathrm{DV}_{10}\right)$, and 
indirectly to assist in the classification of participants’ integration strategies. (See Classification of Participants Integration Strategies).

$D V_{7}$ : Biased usage of hints. Each hint that participants indicated that they had used was coded for the island that the hint favored, +1 for focal (Shell Island) and -1 for non-focal (Coral Island). (A hint favored an island by stating that that Island's current clay pits are characterized by an abundance of this mineral). This produced the seventh dependent variable $\left(\mathrm{DV}_{7}\right)$ biased usage of hints, with a range from +4 , in which every hint favoring Shell Island and no hints favoring Coral Island were used to -4 , in which every hint favoring Coral Island and no hints favoring Shell Island were used. Possible values are all integers between +4 and -4 , because participants could decide to use as many or as few of these hints as they wished. This variable reflects solely usage in integration strategies (not selection in search), since all subjects were presented with all the hints (See Table 15 for Coding).

Table 15

Coding for Biased Usage of Hints

\begin{tabular}{lc}
\hline Direction of Hint & Code \\
\hline Hint Favors Shell Island & +1 \\
Hint Favors Coral Island & -1 \\
\hline
\end{tabular}

$D V_{8}$ : Hypothesis-focus integration bias. Each conditional probability that participants indicated that they had used was coded for the island on which it was 
conditioned, +1 for focal (Shell Island) and -1 for non-focal (Coral Island). This produced the eighth dependent variable $\left(\mathrm{DV}_{8}\right)$ hypothesis-focus integration bias, with a range from +4 , in which every probability used in integration was conditioned on the focal island to -4 , in which every choice was conditioned on the non-focal island. Possible values are all integers between +4 and -4 . This variable reflects the combined effects of participants' integration and search strategies, since the conditional probability must first be selected in the search phase prior to its use in the integration phase (See Table 16 for Coding).

Table 16

Coding for Hypothesis-Focus Integration Bias

\begin{tabular}{lcc}
\hline Direction of Hint & $p$ Conditioned & $p$ Conditioned \\
& on Shell Island & on Coral Island \\
& & \\
\hline Hint Favors Shell Island & +1 & -1 \\
Hint Favors Coral Island & +1 & -1 \\
\hline
\end{tabular}

$D V_{9}$ : Predictor-focus integration bias. Each conditional probability that participants indicated that they had used was coded for the island that the subject mineral was hinted to favor, +1 for focal (Shell Island) and -1 for non-focal (Coral Island). This produced the ninth dependent variable $\left(\mathrm{DV}_{9}\right)$ predictor-focus integration bias, with a range from +4 , in which every choice was of a conditional probability that 
was hinted to favor the focal island to -4 , in which every choice was hinted to favor the non-focal island. Possible values are all integers between +4 and -4 . This variable reflects the combined effects of participants' integration and search strategies, since the conditional probability must first be selected in the search phase prior to its use in the integration phase (See Table 17 for Coding).

Table 17

Coding for Predictor-Focus Integration Bias.

\begin{tabular}{lcc}
\hline Direction of Hint & $p$ Conditioned & $p$ Conditioned \\
& on Shell Island & on Coral Island \\
\hline Hint Favors Shell Island & +1 & +1 \\
Hint Favors Coral Island & -1 & -1 \\
\hline
\end{tabular}

$D V_{10}:$ Pairs used in integration. The conditional probabilities that each participant indicated having used ( 0 to 4 of the 4 conditional probabilities they previously selected) were recoded for the number of diagnostic pairs they constituted. The number of pairs used constitutes the tenth dependent variable $\left(\mathrm{DV}_{10}\right)$ pseudodiagnostic integration, with possible values of 0,1 , and 2 . This variable reflects the combined effects of participants' search and integration strategies, since a conditional probability must first be selected in the search phase prior to its use in the integration phase. 
$D V_{14}:$ Surprise. Surprise was based on a question on the fifth page of the script: "Did you find the results of any of the tests on the old urns surprising? (Yes / No)”. The measurement of this variable is vulnerable to hindsight bias (Fischhoff \& Beyth-Marom, 1975). Unfortunately, it must be asked after the fact, because to ask it before the results of information search are revealed would bias both search and integration strategies. This variable additionally serves as a manipulation check on $\mathrm{IV}_{4}$ : size-of-conditionals, since low conditional probabilities are expected to be surprising.

\section{Operational Definitions of Derived Dependent Variables}

Several dependent measures were calculated and rated on the basis on participants responses. The processes used to create these variables are described below.

Operational definition of $\mathbf{D V}_{4}$ : search strategy. Each participant's search strategy $\left(\mathrm{DV}_{4}\right)$ was derived by Excel if/then statements from their conditional probabilities selections. Figure 1 divides the possible strategies by the number of cells (or quadrants) selected, then by the different patterns these strategies can have, and lastly by whether or not the strategy is Bayesian (includes two matching pairs). First, participants were divided into Bayesians and non-Bayesians (fifth row in Figure 1). Participants who selected two diagnostic pairs (the maximum) with their four selections of conditional probabilities were considered Bayesians, all other participants, whether they select one pair or no pairs, were considered non-Bayesians. 
Method 88

For Bayesians, there are three possible strategies for selecting two diagnostic pairs from two categories (here, pairs favoring Shell or pairs favoring Coral), when selection order and specific selection within a category are not considered: two favoring Shell Island (in the second strategy group from left in the bottom of Figure 1), two favoring Coral Island (in the second strategy group from left in the bottom of Figure 1), or one favoring each island (in the second strategy group from right in the bottom of Figure 1). 
Figure 1. Classification of search strategies by the number of cells used and the use of matched pairs.

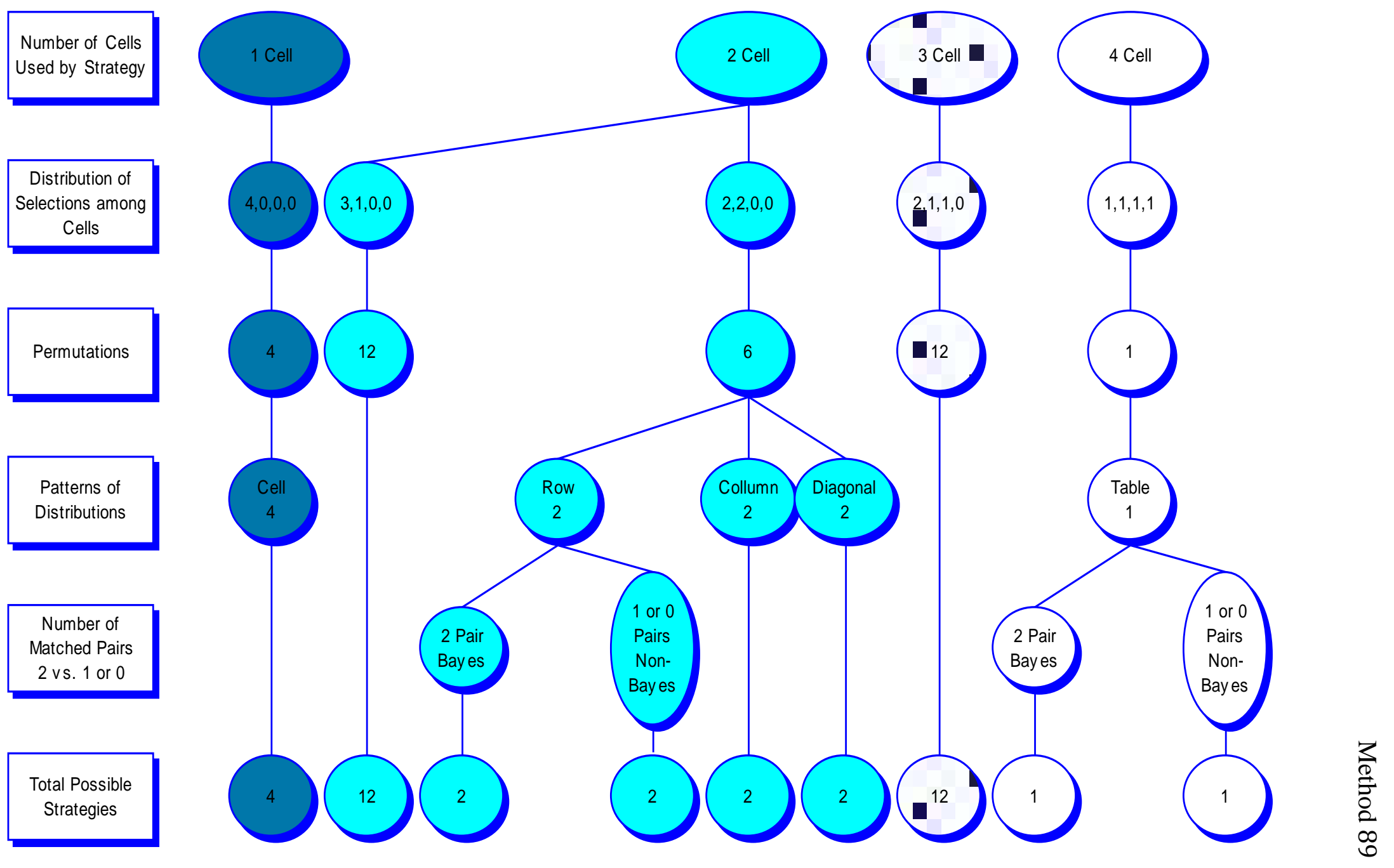


Method 90

For non-Bayesian participants, there are 35 possible strategies for selecting four conditional probabilities from the four quadrants with four members each, when selection order, specific selection within a category, and number of diagnostic pairs are not considered. These categories are defined in terms of the island on which the probability was conditioned and the island that was favored by the hint. Of these 35 strategies, the following 11 are of interest: a) selecting all four choices from one category (Cell Bias, 4 strategies, the left most strategy group in the bottom of Figure 1), b) selecting two choices each from two categories (Column, Row or Diagonal Bias, all 2-2 strategies, 6 strategies, the third through fifth strategy groups from the left in the bottom of Figure 1), and c) selecting one choice from each of the four categories (Table Focus, unbiased, 1 strategy, the right most strategy group in the bottom of Figure 1).

The remaining 24 non-Bayesian strategies would not seem to be of interest, because they do not provide a clear indication about the intent of the participant. These are a) selecting three choices from one category and one choice from another category (3-1 class of strategies, 12 strategies, third from the right strategy group in the bottom of Figure 1), and b) selecting two choices from one category and one choice each of two other categories (2-1-1 class of strategies, 12 strategies, fourth from the right strategy group in the bottom of Figure 1). These 24 strategies would not seem to be of interest for two reasons: participant reliability and generalizability of strategy. First, these 24 strategies provide less evidence of participant reliability. A strategy is deemed to be one that is reliably used by the participant if either all the categories 
Method 91

involved are selected at least twice, or if diagnostic pairs are selected at least twice. All the strategies of interest meet this criterion, except the Table Focus, which was included because it represents the only unbiased non-Bayesian strategy (and it is also the only strategy with no redundancy). On the basis of this criterion alone, one could eliminate the 24 strategies deemed not of interest. Second, these same strategies would be eliminated by another criterion: They cannot be defined by a simple rule that can be easily generalized to other situations. For example, when picking eight of 32 conditional probabilities, it is clear that a one-category strategy (4-0-0-0) would pick all eight conditional probabilities from the same category (8-0-0-0); however, it is unclear whether someone using the 2-1-1-0 strategy would choose a 3-3-2-0, 4-2-2-0, 5-1-1-1, or a 6-1-1-0 strategy.

There are thus 14 identified search strategies, and one default category that were coded. These can be grouped into five classes: Bayesian, One-Category, TwoCategory, Four-Category, and Unclassifiable (2-1-1 and 3-1).

\section{Bayesian strategies.}

1 Bayesian unbiased (Optimal): Two pairs, one pair hinted to be in each direction.

2 Bayesian Confirmatory Predictor Bias: Two pairs, both hinted to favor Shell Island (a row bias pattern).

3 Bayesian Disconfirmatory Predictor Bias: Two pairs, both hinted to favor Coral Island (a row bias pattern). 


\section{Non-Bayesian one-category strategies (cell bias).}

4 Shell Island both Focal and Motivated: Four conditional probabilities hinted to favor Shell Island and conditioned on Shell Island.

5 Shell Island Focal and Coral Island Motivated: Four conditional probabilities hinted to favor Coral Island but conditioned on Shell Island.

6 Coral Island Focal and Shell Island Motivated: Four conditional probabilities hinted to favor Shell Island but conditioned on Coral Island.

$7 \quad$ Coral Island both Focal and Motivated: Four conditional probabilities hinted to favor Coral Island and conditioned on Coral Island.

\section{Non-Bayesian two-category strategies (column, row or diagonal bias).}

8 Shell Island Column Focal: Four probabilities conditioned on Shell Island, with two hinted to favor Shell Island and two hinted to favor Coral Island (Column Bias).

9 Coral Island Column Focal: Four probabilities conditioned on Coral Island, with two hinted to favor Shell Island and two hinted to favor Coral Island (Column Bias).

10 Shell Island Row Motivated: Four probabilities conditionals hinted to favor Shell Island, with two conditioned on Shell Island and two conditioned on Coral Island (Row Bias).

11 Coral Island Row Motivated: Four probabilities conditionals hinted to favor Coral Island, with two conditioned on Shell and two conditioned on Coral Island (Row Bias). 
Method 93

12 Consistent Diagonal: Two conditional probabilities both hinted to favor Shell Island and conditioned on Shell Island, and two conditional probabilities both hinted to favor Coral Island and conditioned on Coral Island.

13 Inconsistent Diagonal: Two conditional probabilities hinted to favor Shell Island but conditioned on Coral Island, and two conditional probabilities hinted to favor Coral Island but conditioned on Shell Island.

\section{Non-Bayesian four-category strategy (table focus, unbiased).}

14 Sampling Strategy: One selected from each category.

\section{Unclassifiable strategies.}

15 Unclassifiable Strategies. This includes the 12 three-category strategies (2-11), and the 12 uneven two-category strategies (3-1).

\section{Classification of search strategies as focal and predictor biased. These} strategies can be further coded as focal, neutral or anti-focal. Strategies that include all four probabilities conditioned on Shell were coded as focal (+1). Strategies that include all four probabilities conditioned on Coral were coded as anti-focal (-1). All other strategies were coded as neutral (0). Similarly, these strategies can be further coded as and as predictor biased, neutral or anti-predictor biased. Strategies that include all four conditional probabilities on dimensions hinted to favor Shell were coded as predictor-biased (+1). Strategies that include all four conditional probabilities on dimensions hinted to favor Coral were coded as anti-predictor biased (-1). All other strategies were coded as neutral (0). These two codes were summed and trichotomized 
Method 94

$(+, 0,-)$ into an overall bias, neutral unbiased search strategies (See Table 18 for a list of search strategies with their focal and predictor codes). 
Table 18

Coding of Search Strategies as Focal and Predictor Biased

\begin{tabular}{|c|c|c|c|}
\hline \multirow[t]{2}{*}{ Strategy Class } & \multirow[t]{2}{*}{ Strategy } & \multirow{2}{*}{$\begin{array}{l}\text { Focal } \\
\text { Code }\end{array}$} & \multirow{2}{*}{$\begin{array}{l}\text { Predictor } \\
\text { Code }\end{array}$} \\
\hline & & & \\
\hline \multirow[t]{3}{*}{ Bayesian Strategies } & Bayesian Unbiased & 0 & 0 \\
\hline & Bayesian Confirmatory Predictor Bias & 0 & +1 \\
\hline & Bayesian Disconfirmatory Predictor Bias & 0 & -1 \\
\hline Non-Bayesian One- & Shell Island both Focal and Motivated & +1 & +1 \\
\hline \multirow[t]{5}{*}{ Category Strategies } & Shell Island Focal and Coral Island & +1 & -1 \\
\hline & Motivated & & \\
\hline & Coral Island Focal and Shell Island & -1 & +1 \\
\hline & Motivated & & \\
\hline & Coral Island both Focal and Motivated & -1 & -1 \\
\hline Non-Bayesian Two- & Shell Island Column Focal & +1 & 0 \\
\hline \multirow[t]{5}{*}{ Category Strategies } & Coral Island Column Focal & -1 & 0 \\
\hline & Shell Island Row Motivated & 0 & +1 \\
\hline & Coral Island Row Motivated & 0 & -1 \\
\hline & Consistent Diagonal & 0 & 0 \\
\hline & Inconsistent Diagonal & 0 & 0 \\
\hline Non-Bayesian Four- & Sampling Strategy & 0 & 0 \\
\hline \multicolumn{4}{|l|}{ Category Strategy } \\
\hline Unclassifiable & Unclassifiable Strategies & Not & Not \\
\hline Strategies & & Coded & Coded \\
\hline
\end{tabular}


Method 96

Operational definition of $\mathbf{D V}_{11}$ : integration strategy. The strategies in this classification system were selected to achieve the goals of classifying participants by the conditional probabilities they used and by the identifiable mathematical formula (if any) they used to calculate their posterior probabilities. The strategies in the initial version of the classification system came from three sources: a) the integration strategies found in a pilot study (Borthwick \& Anderson, 2001, Appendix E); b) strategies of participants in the current study who wrote out their strategies mathematically; c) several additional, non-mathematical strategies the author felt possible. In a test of the classification system was performed on a small sample of the current participants, the non-mathematical strategies were found to be difficult to classify reliably. Therefore, these non-mathematical strategies were collapsed into 3 strategies, producing the final classification system of 14 strategies (11 mathematical and 3 non-mathematical). All strategies are defined below.

The final classification system was used by two raters who independently classified each participant into one of the fourteen integration strategies (seven of which are subdivided by which island they focus on). In cases where the two raters disagreed, they discussed and determined the appropriate rating jointly. The joint decision of the raters then became the eleventh dependent variable $\left(\mathrm{DV}_{11}\right)$ Integration Strategy.

Two measures were used to assess inter-rater reliability. The first is the percentage of agreement, which will be measured as a percentage of all ratings that are in agreement (72.8\%). The second is Cohen’s (1960) Kappa, which has the desirable 
property that it takes into account the effects of chance agreement (Kappa $=.687$, Standard Error $=.029)$.

To classify participants’ integration strategies, the two raters used the following information, listed in decreasing order of importance: a) posterior probability, b) information search selections (both the values of the conditional probabilities and the availability of Bayesian matched pairs), c) mathematical computations (if any), d) verbal descriptions of integration strategies (question 1 on page 5), e) indications of information used ( $\mathrm{DV}_{7}$ through $\left.\mathrm{DV}_{10}\right)$ in calculating the final probability estimate, and f) the mathematical operations indicated as having been used (question 2 on page 5).

The first of these items that the raters used was whether each participant selected in the search phase at least one diagnostic pair (Bayesian) or no diagnostic pairs (Non-Bayesian). The second of these items was whether an identifiable mathematical formula could be specified. All the above information was used to determine a mathematical formula that could account for the participant's revised probability estimate given the information available to him or her.

There were two items of information that could have been used, but were generally not used, to classify a participant's integration strategy. The first of these was which, if any, boxes a participant checked to indicate the dimensional hints (the abundance of each mineral in the modern clay pits of the two islands) used in integration. While the hints were designed to bias the selection of information, they were ignored here (in integration) for two reasons. First, any use of these hints should 
Method 98

have had no effect on integration, because the hints are non-numeric and balanced. Second, there is reason to believe that some participants who indicated that they had used the hints in integration actually were indicating that they had used the hints in selection. (The reason for the latter belief is that what each hint provides information about is the values of the two conditional probabilities on a single dimension. Many of the participants who indicated that they had used a dimensional hint in integration already knew the exact value of both of the dimension's conditional probabilities. In this case, knowledge of the value of the conditional probabilities makes the hint superfluous in integration).

The second item of information generally not used was whether participants indicated having used the base rate. This item of information was not usually considered because the base rate was even and therefore likely to be ignored by most participants and, if not ignored, unlikely to have had an impact on the posterior probability. However, if a participant provided multiple indications of having used a non-Bayesian integration strategy that included the base rate as a critical part, then the fact that a participant indicated having used the base rate was taken into account. There were only two such strategies: a) base rate + largest conditional, and b) base rate.

The fourteen strategies are listed below (see Figure 2) and described in sections: Mathematical Two-Island Bayesian Strategies, Mathematical Two-Island Non-Bayesian Strategies, Mathematical One-Island Strategies, Mathematical ZeroIsland Strategy, and Non-Mathematical Strategies. These strategies are categorized 
Method 99

into those with an identifiable mathematical formula (11 strategies) and those with no identifiable mathematical formula (3 strategies). Although the distinction between mathematical and non-mathematical is useful in categorizing these strategies, the first step towards categorizing participants was determining whether their search strategies did or did not provide diagnostic pairs (that is, whether they searched diagnostically), as required by Bayes’ Theorem. The determination of Bayesian search comes first, because there are three integration strategies used exclusively by participants who searched diagnostically, two of which are mathematical (Bayesian, Biased Bayesian) and one non-mathematical (Diagnostic Search with Default Integration).

To summarize, the entire classification process was as follows: First, an attempt was made to match participants to a Mathematical Bayesian Strategy; failing that, an attempt was made to match them to a Mathematical Non-Bayesian Strategy; failing both, participants were matched to one of the three Non-Mathematical Strategies. 
Figure 2. Categorization of integration strategies by the information and math used.

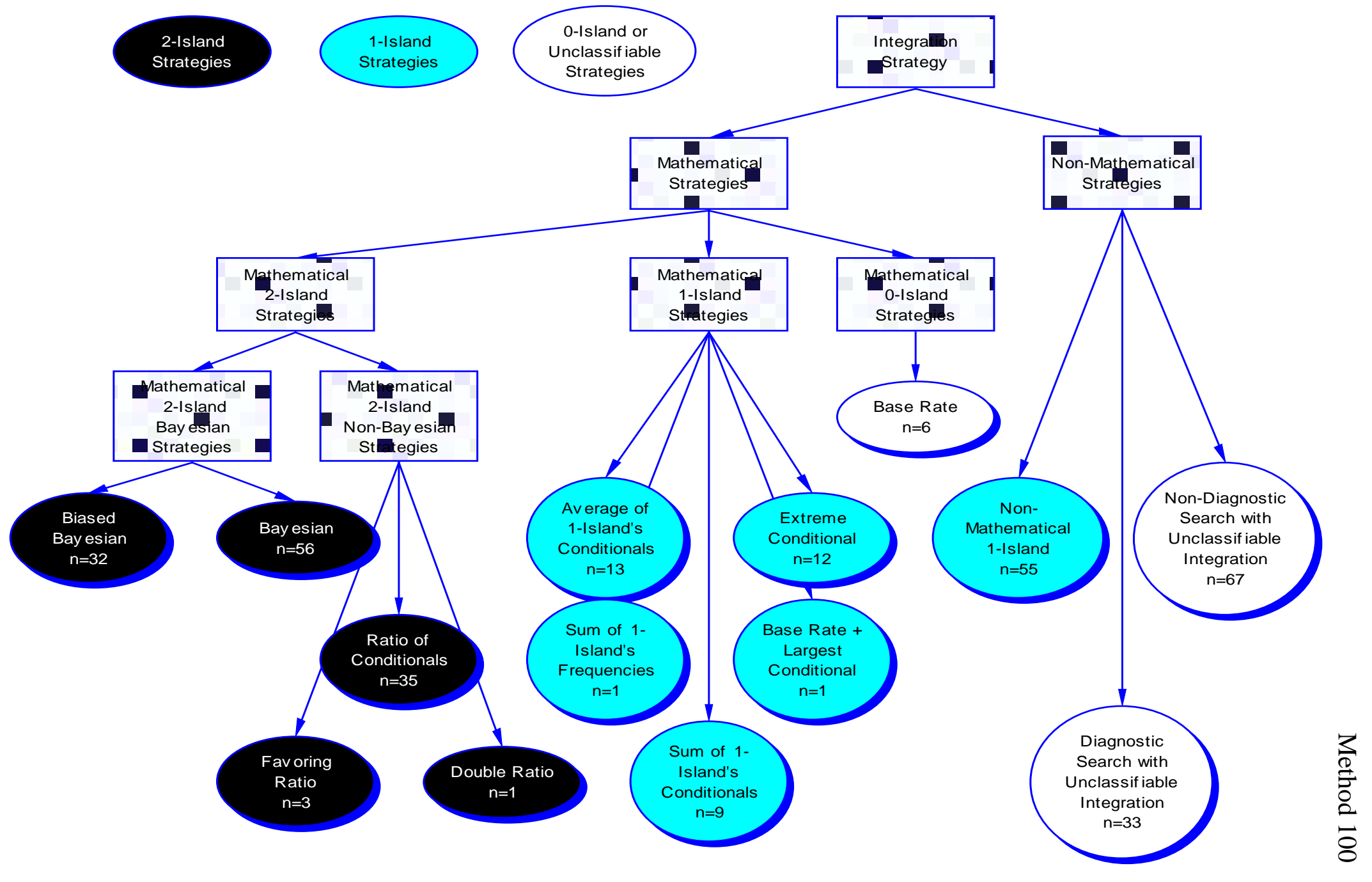


Method 101

The eleven mathematical strategies are sub-divided according to whether the formula uses probabilities conditioned on one or both islands or uses no conditional probabilities. Subjects were classified as showing one of the 11 mathematical strategies if they met two main criteria. The primary criterion was that participants must have produced a posterior probability within a range of $+/-5 \%$ of the posterior produced by using the conditional probabilities available to them and the formula of the relevant mathematical strategy. This criterion was modified in two cases.

The first case where the primary criterion was modified was the two mathematical Bayesian strategies. The requirement that the posterior fall within $a+/$ $5 \%$ range centered on the correct posterior was changed to the requirement that it fall within a rounded range (the centered range rounded to the nearest 5\%). In this case, the change was made to facilitate rater comprehension. This change was practical for Bayesian strategies and only Bayesian strategies, since they are the only strategies that (because of the fixed 2:1 diagnostic ratio) permit only a very limited number of correct outcomes (three outcomes and their complements). This made it possible to calculate all the possible posteriors before conditional probabilities had been selected. (The three possible correct rounded ranges are as follows. 1) If the participant had selected and used in integration two pairs favoring the same island, the correct Bayesian posterior would be $80 \%$ ( $20 \%$ for the other island) and the rounded range would be 75-85\%. 2) If the participant had selected one or two pairs and used one pair in integration, the correct Bayesian posterior would be $66.66 \%$ (33.33\% for the other island) and the rounded range would be $60-70 \%$. 3) If the participant had selected two 
pairs favoring different islands and used both, the correct Bayesian posterior would be 50\%, and the rounded range was $45-55 \%$.]

The second case where the primary criterion was modified was the base rate strategy. The primary criterion (allowing a +/- 5\% range) was changed to require a posterior of exactly 50\%, for two reasons. First, because many strategies can result in a revised probability of approximately $50 \%$, the range of $45 \%-55 \%$ would not be very diagnostic. Second, because this strategy requires no calculating, there is no reason to allow for errors in calculation.

The secondary criterion (for classifying integration strategies) was some verbal or mathematical evidence of having used the strategy. This criterion required only minimal mathematical or verbal indication supporting the participant's use of the strategy. This criterion was modified in four cases.

The first case where the secondary criterion was modified was when participants estimated a posterior of exactly 50\%. In this case the minimal standard was made more stringent (because a 50\% posterior can result from many strategies). In this case, the second criterion was changed to require that the participant have either written out the complete mathematical formula or have made an explicit verbal statement of the strategy.

The second case where the secondary criterion was modified was for the two Bayesian strategies. Because the formula is complex and hard to distinguish if not completely spelled out, the secondary criterion was changed to require the participants 
either to spell out the formula completely or at least not to specify any computation inconsistent with Bayes’ formula.

The third case where the secondary criterion was modified was for the base rate strategy. The secondary criterion was tightened to require that the participants must not have shown any indication of having used any conditional probabilities (no checks on boxes corresponding to conditional probability evidence or mathematical writings including the values of conditional probabilities), and they must have either checked the box indicating that they used the base rate or mentioned using the base rate in their verbal description.

The fourth case where the secondary criterion was modified was for the double ratio and sum of frequencies strategies. Because these strategies were unexpected, the secondary criterion was tightened to require that participants must have produced mathematical writings calculating this formula. (These strategies were used by only one subject each, but they were included because those two subjects were very specific and clear in stating their mathematical calculations used.)

All the modifications from the two main criteria are noted in Table 19 below as well as in the relevant strategy descriptions. 
Table 19

Exceptions to the Two Main Criteria for Classification to an Integration Strategy

\begin{tabular}{|l|l|l|}
\hline Mathematical Strategy & Exception to Primary & Exception to Secondary \\
& Criterion (posterior & Criterion (evidence of \\
\hline Bayesian & Rounded Range & Moving used the strategy) \\
\hline Biased Bayesian & Rounded Range & More explicit or not \\
\hline Ratio of Conditionals & & inconsistent \\
\hline Favoring Ratio & & Mustent \\
\hline Double Ratio & & Mow math \\
\hline Sum of One-Island's Conditionals & & \\
\hline Sum of One-Island's Frequencies & & \\
\hline Average of One-Island's & & \\
\hline Conditionals & & \\
\hline Extreme Conditional & & \\
\hline Base Rate + Largest Conditional & & \\
\hline
\end{tabular}


Method 105

There are five mathematical two-island strategies. These are sub-divided into two Bayesian and three Non-Bayesian strategies.

Mathematical two-island Bayesian strategies (2 strategies). The two Bayesian mathematical strategies (Biased Bayesian and Bayesian) will be described together. These both require that participants have first searched diagnostically (selecting one or two diagnostic pairs in the search phase) in order to be considered for classification. Both of the two main criteria were modified for these strategies as discussed above. For both participants must have produced a posterior within a rounded range of the correct posterior (criterion \#1) using at least one diagnostic pair and must either have written out Bayes formula or at least made no indications contrary to Bayes formula (criterion \#2).

In order to distinguish between Biased Bayesian and Bayesian it is clearer to describe the more specific strategy first (Biased Bayesian). There are three different ways to search for one or two diagnostic pairs in this scenario: one pair, two pairs favoring the same island, or two pairs with one hinted to favor each island (optimal search as demonstrated above under "Demonstration of the Optimal Search and Integration Strategies”). Biased Bayesian participants were those who searched optimally but used in integration only one of the two available pairs correctly (that is a posterior within a rounded range, criterion \#1) and who met the requirements of the second criterion. Biased Bayesian participants were further distinguished by which of the two islands they were biased towards. 
Participants who did not qualify for Biased Bayesian would be classified as Bayesian if they fulfilled three criteria: 1) They produced one or two diagnostic pairs in the search phase. 2) They produced a posterior probability within the rounded range (criterion \#1) of the correct posterior for using one or both of the pairs available to them. 3) They met the requirements of the second criterion.

There are two differences between the two Bayesian integration strategies. The first difference is in the search phase requirements. Bayesian integration requires that the participant obtain at least one pair in integration, so that they can integrate at least one pair using Bayes’ theorem. Biased Bayesian integration requires that participants have searched optimally (two pairs with one hinted to favor each island). This is the only way to search that enables participants both to integrate using Bayes Theorem and yet to show a bias through their choice of which of the two pairs to use in integration. Second, although both Bayesian integration strategies require the posterior be within a rounded range, in Biased Bayesian integration the range is built around the correct use of only one pair, while in Bayesian integration the range is built around correctly using either one or two pairs.

Example used to illustrate all mathematical strategies. All remaining mathematical strategies will be illustrated by means of the example below. Under each mathematical strategy the correct mathematical formula used to compute the posterior probability (assuming the results of search shown in Table 20) is provided as an illustration. This would be the posterior that the participant would have to come within $5 \%$ of in order to satisfy the first criterion for being classified with that strategy. In this 
example the hypothetical participant selected one conditional probability from each quadrant.

Table 20.

Example used for all Mathematical Strategies

\begin{tabular}{lll}
\hline Direction of Hint & $p$ Conditioned on Shell & $p$ Conditioned on Coral \\
& Island & Island \\
\hline Hint Favors Shell Island & $80 \%$ or 16 Frequency & $40 \%$ or 8 Frequency \\
Hint Favors Coral Island & $30 \%$ or 6 Frequency & $60 \%$ or 12 frequency \\
\hline
\end{tabular}

This example would result in a 50\% posterior using Bayesian integration if the four conditional probabilities formed either two or no matching pairs.

Mathematical Two-Island Non-Bayesian Strategies (3 Strategies). There were three identifiable mathematical non-Bayesian strategies that use evidence from both islands in the classification system. All three use all available information, are unbiased in their computation, and are symmetric regarding the two islands, making the island on which the calculation is based on irrelevant. However, while the computation is unbiased, bias can be introduced in the search phase. The mathematical formulas for the three Mathematical Two-Island Non-Bayesian Strategies follow, each in their own section.

Ratio of conditionals. The formula for ratio of conditionals is: 
$\sum$ (probabilities conditioned on one island) / $\sum$ (all conditional probabilities).

Using the example above, the calculation would be:

Posterior probability if calculated on Shell $=(80 \%+30 \%) /(80 \%+30 \%+40 \%+60 \%)=$ $52.38 \%$.

Posterior probability if calculated on Coral $=(40 \%+60 \%) /(80 \%+30 \%+40 \%+60 \%)=$ $47.62 \%$.

Favoring ratio. The formula for favoring ratio is:

$\sum$ (conditional probabilities hinted to favor one island) $/ \sum$ (all conditional probabilities).

Using the example above, the calculation would be:

Posterior probability if calculated on Shell $=(80 \%+40 \%) /(80 \%+30 \%+40 \%+60 \%)=$ $57.14 \%$

Posterior probability if calculated on Coral $=(30 \%+60 \%) /(80 \%+30 \%+40 \%+60 \%)=$ $42.86 \%$.

Double ratio. The formula for double ratio is:

$\left[\sum\right.$ (probabilities conditioned on Shell) $/ \sum$ (all conditional probabilities) $]+$ [ $\sum$ (probabilities hinted to favor Shell) $/ \sum$ (all conditional probabilities)]/2. Using the example above, the calculation would be: Posterior probability if calculated on Shell $=[(80 \%+30 \%) /(80 \%+30 \%+40 \%+60 \%)+$ $(80 \%+40 \%) /(80 \%+30 \%+40 \%+60 \%)] / 2=54.76 \%$

Posterior probability if calculated on Coral $=[(40 \%+60 \%) /(80 \%+30 \%+40 \%+60 \%)+$ $(30 \%+60 \%) /(80 \%+30 \%+40 \%+60 \%)] / 2=45.23 \%$ 
Method 109

This strategy can be thought of as the average of the other two mathematical two-island non-Bayesian strategies. The secondary criterion was tightened for this strategy as noted above.

Mathematical one-island strategies (5 strategies). There were five identifiable mathematical strategies using evidence from only one island in the classification system. These strategies are not symmetric, in that it matters which island the participant focuses the computation on. Therefore, all five of these strategies were further subdivided into whether the computation used probabilities conditioned on Shell or Coral. The mathematical formulas for the five Mathematical One-Island Strategies follow, each in their own section.

Sum of one-island's conditionals. The formula for sum of one-island's conditionals is:

$\sum$ (probabilities conditioned on one of the islands)

Using the example above, the calculation would be:

Posterior probability if calculated on Shell $=(80 \%+40 \%)=120 \%$.

Posterior probability if calculated on Coral $=(30 \%+60 \%)=90 \%$.

Sum of one-island's frequencies. The formula for sum of one-island's frequencies is:

$\sum$ (frequencies about one of the islands). This number is then treated as a probability.

Using the example above, the calculation would be:

Posterior probability if calculated on Shell $=(16+8)=24$, which was treated as $24 \%$. 
Posterior probability if calculated on Coral $=(6+12)=18$, which was treated as $18 \%$.

The secondary criterion was tightened for this strategy as noted above.

Average of one-island's conditionals. The formula for average of one-island's conditionals is:

$\sum$ (probabilities conditioned on one of the islands) / n

Using the example above, the calculation would be:

Posterior probability if calculated on Shell $=(80 \%+30 \%) / 2=55 \%$.

Posterior probability if calculated on Coral $=(40 \%+60 \%) / 2=50 \%$.

Extreme conditional. The formula for extreme conditional is: The value of the single most extreme conditional.

Using the example above, the calculation would be:

Posterior probability if calculated on Shell $=80 \%$

Posterior probability if calculated on Coral $=$ not possible in the example case because the most extreme probability is conditioned on Shell.

Base rate + largest conditional. The formula for base rate plus largest conditional is:

Base Rate + Largest Conditional Probability

Using the example above, the calculation would be:

Posterior probability if calculated on Shell $=(80 \%+50 \%)=130 \%$. This was treated as $100 \%$.

Posterior probability if calculated on Coral $=$ not possible in the example case because the largest probability is conditioned on Shell. 
Method 111

Mathematical zero-island strategy (1 strategy). There was only one integration strategy that used an identifiable mathematical formula in the classification system. It used just the base rate, but no conditional probability evidence.

Base rate. The formula for base rate is: The base rate (50\% in this study).

For example, if the available conditional probabilities were: $80 \%$ conditioned and hinted to favor Shell, 60\% conditioned on Shell and hinted to favor Coral, $40 \%$ conditioned on Coral but hinted to favor Shell, and 30\% conditioned on and hinted to favor Coral. Then the calculation would be:

Base Rate $=50 \%$

Because a 50\% posterior can be produced in many ways, the criteria for the base rate strategy were more stringent than the others. The primary criterion was tightened to require that the posterior equal the base rate (50\%) exactly (as discussed above under Mathematical Strategies). The secondary criterion was tightened to require that the participants must not have shown any indication of having used any conditional probabilities (no checks on boxes corresponding to conditional probability evidence or mathematical writings including the values of conditional probabilities), and they must have either checked the box indicating that they used the base rate or mentioned using the base rate in their verbal description.

Non-mathematical strategies (3 strategies). All participants who did not have an identifiable mathematical strategy were classified as having one of three nonmathematical strategies (described below). Where possible, the conditional probabilities the participant used in integration were identified. Any minimal 
indication as to the island on which probabilities they used were conditioned was taken as a basis to classify them as using information from that island. Acceptable indications included: a) any checkmark indicating a conditional probability used; b) any written number corresponding (in either probability or frequency form) to any conditional probability they received; and / or, c) any verbal description implying the use of a conditional probability. This minimal standard was used for all nonmathematical strategies.

Non-mathematical one-island. If a non-diagnostic participant did not have a mathematical strategy but did produce an indication (see above) of having used one or more probabilities all conditioned on the same island, then they were classified as nonmathematical one-island. This strategy was subdivided according to which of the two islands the data used was conditioned on (identical to the one-island mathematical strategies).

Non-diagnostic search with unknown integration. This was the default category for all participants who searched non-diagnostically. These participants did not have a mathematical strategy, and did not clearly indicate having used probabilities all conditioned on the same island.

Diagnostic search with unknown integration. This was the default category for all participants who searched diagnostically. Because these participants were diagnostic in their search, it was assumed in the absence of any clear indication to the contrary, that they at least used probabilities conditioned on both islands in their integration. 
Method 113

Classification of integration strategies as focal and predictor biased. These integration strategies can be further coded as focal, neutral or anti-focal. Strategies that include probabilities conditioned only on Shell were coded as focal $(+1)$. Strategies that include only probabilities conditioned only on Coral were coded as anti-focal (-1). All other strategies were coded as neutral (0). Similarly, these strategies can be further coded as and as predictor biased, neutral or anti-predictor biased. Strategies that include conditional probabilities only on dimensions hinted to favor Shell were coded as predictor-biased $(+1)$. Strategies that include conditional probabilities only on dimensions hinted to favor Coral were coded as anti-predictor biased (-1). All other strategies were coded as neutral (0). These two codes were summed and trichotomized $(+, 0,-)$ into an overall bias, neutral unbiased integration strategies (See Table 21 for a list of integration strategies with their focal and predictor codes). 
Table 21

Coding of Integration Strategies as Focal and Predictor Biased

\begin{tabular}{|c|c|c|c|}
\hline \multirow[t]{3}{*}{ Strategy Class } & \multirow[t]{3}{*}{ Strategy } & \multirow{2}{*}{$\begin{array}{l}\text { Focal Code } \\
\text { (Shell / }\end{array}$} & \multirow{2}{*}{$\begin{array}{l}\text { Predictor } \\
\text { Code (Shell }\end{array}$} \\
\hline & & & \\
\hline & & Coral) * & / Coral) * \\
\hline Mathematical & Biased Bayesian & $(0 / 0)$ & $(+1 /-1)$ \\
\hline \multirow[t]{4}{*}{ 2-Island } & Bayesian & 0 & 0 \\
\hline & Ratio of Conditionals & 0 & 0 \\
\hline & Favoring Ratio & 0 & 0 \\
\hline & Double Ratio & 0 & 0 \\
\hline Mathematical & Sum of One-Island's Conditionals & $(1 /-1)$ & $(0 / 0)$ \\
\hline \multirow[t]{4}{*}{ 1-Island } & Sum of One-Island's Frequencies & $(1 /-1)$ & $(0 / 0)$ \\
\hline & Average of One-Island's Conditionals & $(1 /-1)$ & $(0 / 0)$ \\
\hline & Extreme Conditional & $(1 /-1)$ & $(1 /-1)$ \\
\hline & Base Rate + Largest Conditional & $(1 /-1)$ & $(1 /-1)$ \\
\hline Mathematical & Base Rate & 0 & 0 \\
\hline \multicolumn{4}{|l|}{0 -Island } \\
\hline Non- & Non-Mathematical One-Island & $(1 /-1)$ & $(1 /-1)$ \\
\hline \multirow[t]{3}{*}{ Mathematical } & $\begin{array}{l}\text { Non-Diagnostic Search with Unknown } \\
\text { Integration }\end{array}$ & Not Coded & Not Coded \\
\hline & Diagnostic Search with Unknown & Not Coded & Not Coded \\
\hline & Integration & & \\
\hline
\end{tabular}


* Note: Six strategies were defined by whether the probabilities used were conditioned on Shell or Coral and the Biased Bayesian Strategy was defined by whether the bias favored Shell or Coral. For these seven strategies the coding is split into the codes for the Shell or Coral version of that strategy.

\section{Operational definition of $\mathrm{DV}_{6}$ : search confirmation bias. Search}

confirmation bias was calculated by subtracting the normative revised probability estimate that the Urn had been created on Shell Island (.50 in this scenario) from the revised probability applying Bayes’ Theorem to the conditional probabilities actually selected by each participant. This compares two normative posteriors, one based on a normative search and one based on the actual pairs (if any) selected by the participant. Each pair chosen was coded +1 if it favored Shell Island (that is, the corresponding hint stated that the current clay pits on Shell Island are characterized by an abundance of this mineral), and -1 if it favored Coral Island, producing a sum with the possible values of $+2,+1,0,-1$, and -2 . For each of these five sums, the application of Bayes' Theorem produces the corresponding following five posterior probabilities .8, .667, .5, .333, and .2, respectively. Since all pairs have the same diagnostic ratio (2:1) it does not matter which of the four pairs in a particular direction a participant chose. If participants chose one in each direction the net effect would cancel out.

Next, the optimal revised probability estimate (.50) was subtracted from the five posterior probabilities listed above, producing the five possible values for search confirmation bias (+.3, +.167, 0, -.167, -.3). For example, if Bayes’ Theorem were applied to the conditional probabilities selected by a participant and this produced a 
Method 116

revised estimate of.8, then his or her search confirmation bias score would be .3 (.8 $.5=.3$ ). These values are the bias caused by the participant's search phase when integration is unbiased.

For most participants, the revised probability created from each participant's actual search using Bayes' theorem was the same as the base rate, because most ( $n=219)$ people searched pseudodiagnostically (as first demonstrated by Doherty, et al., 1979). (If the search phase is pseudodiagnostic, then there will be no pairs for use in Bayes theorem, and therefore no revision). Therefore, search confirmation bias is the difference between the base rate and the normative revised probability, except when participants do not select one or more pairs. In this specific situation participants with non-Bayesian searches will have no search confirmation bias.

Once again the subtraction of .50 (the normative revised probability) is a linear transformation, that does not alter the statistical properties of the variable, but does make it easier to interpret and compare to the other bias dependent variables.

Operational definition of $\mathrm{DV}_{12}$ : integration confirmation bias. Integration confirmation bias was calculated by subtracting a) the optimal revised probability (.50 in this scenario) from b) the revised probability that would result from combining the optimal search strategy with the participant's integration strategy as classified by $\mathrm{DV}_{11}$ : integration strategy. This compares two posteriors, both of which are based on optimal search (one diagnostic pair in each diagnostic direction), where one of the posteriors is based on Bayesian integration and the other posterior is based on the participant’s inferred integration strategy (DV 11 integration strategy). 
Calculating integration confirmation bias involved three steps. First, the results of an optimal search had to be determined. Because the optimal search produces two diagnostic pairs (one in each diagnostic direction), it yields four conditional probabilities, one from each of the four categories. Thus, any of the four conditional probabilities in each category has an equal chance of being selected. For this reason, the average conditional probability in each category was used to represent the result of the optimal search. This both preserves the diagnostic ratio (2:1) for Bayesians, while providing a value for use by non-Bayesians. This average conditional probability depended on the level of $\mathrm{IV}_{4}$ size-of-conditionals. In the high condition, the average value was .75 for probabilities conditioned on the island favored by the hint and .375 for probabilities conditioned on the island not favored by the hint, and in the Low Condition the averages were .25 and .125 , respectively.

Second, each integration strategy as classified by $\mathrm{DV}_{11}$ : integration strategy was applied to these average conditional probabilities to yield a posterior. This posterior is a continuous variable with a range of possible values from 0 to 1.0. Any resulting posterior outside this possible range was treated as a 0 or 1.0 (See Table 22 for a list of these calculated posteriors). $\mathrm{DV}_{12}$ : integration confirmation bias is the posterior resulting from combining the optimal search strategy with the participant's actual integration strategy. 
Table 22

Posterior Produced by Optimal Search and Each Integration Strategy

\begin{tabular}{lcc}
\hline Integration Strategy & High Conditionals & Low Conditionals \\
\hline Biased Bayes & $66.67 \%$ & $66.67 \%$ \\
Bayes & $50 \%$ & $50 \%$ \\
Ratio of Conditionals & $50 \%$ & $50 \%$ \\
Favoring Ratio & $50 \%$ & $50 \%$ \\
Double Ratio & $50 \%$ & $50 \%$ \\
Sum of Conditionals & $100 \%$ & $37.5 \%$ \\
Sum of Frequencies & $22.5 \%$ & $7.5 \%$ \\
Average of Conditionals & $56.25 \%$ & $18.75 \%$ \\
Extreme Conditional & $75 \%$ & $87.5 \%$ \\
Base Rate + Conditional & $100 \%$ & $75 \%$ \\
Base Rate & $50 \%$ & $50 \%$ \\
Non-Mathematical & $\mathrm{n} / \mathrm{a}$ & \\
\hline
\end{tabular}

Finally, a difference score was computed by subtracting the optimal revised probability estimate (.50) from the posterior calculated above, producing $\mathrm{DV}_{12}$ : Integration Confirmation Bias. This dependent variable has a range from +.50 , meaning the participant's integration strategy favored Shell Island .50 more than optimal (confirmatory bias), to -.50, meaning the participant’s integration strategy favored Coral Island .50 more than optimal (disconfirmatory bias). 
Integration confirmation bias represents the bias caused by the participant's integration strategy alone. However, this dependent variable is not completely independent of the participant's prior search strategy. This prior search strategy is expected to limit the choice of integration strategies in two ways, a) by ruling out integration strategies that require information that is not available, and b) by possibly leading to the rejection of strategies that yield posterior probabilities that are outside the range (0-1.0) or otherwise not believable. (For example, subjects who selected all four conditionals favoring and conditioned on the same island, would receive conditional probabilities of $10 \%, 20 \%, 30 \%$ and $40 \%$ if they were in the low conditionals level of $\mathrm{IV}_{4}$ size-of-conditionals. If these subjects then chose to add the four conditionals together in integration they would produce a believable result $100 \%$. However, if subjects with the same search strategy and integration strategy were in the high conditionals level of $\mathrm{IV}_{4}$ size-of-conditionals, then they would have received conditional probabilities of $60 \%, 70 \%, 80 \%$ and $90 \%$ which if added together would be $300 \%$, presumably forcing the subject to use a different integration strategy).

\section{Operational definition of $\mathrm{DV}_{13}$ : sum-of-phase-biases.}

Sum-of-Phase-Biases is calculated as the sum of the two specific phase biases ( $\mathrm{DV}_{6}$ search confirmation bias and $\mathrm{DV}_{12}$ integration confirmation bias). This produces a continuous variable with a range of +.8 to -.8 . Where +.8 is interpreted to mean the participant's actual search phase integrated by Bayes’ Theorem $\left(\mathrm{DV}_{6}\right.$ search confirmation bias) and their actual integration strategy applied to the results of optimal search ( $\mathrm{DV}_{12}$ integration confirmation bias) sum to a +.8 bias towards making Shell 
Island more likely as the island of origin of the Urn. If the two phase biases are additive, then this variable should equal $\mathrm{DV}_{4}$ : Confirmation Bias. This would lead to the conclusion that search and integration are separate systems, completely independent of each other (system defined by Lendaris, 1986). Significant departures from additivity are expected and will be discussed.

Operational definition of $\mathbf{D V}_{15}$ : intuitive conditionals. Participants were asked on the fifth page "Please go back to page three again and, without removing any more stickers, write down on the stickers what you think the value of the tests would be that you did not choose to perform.” These values were recorded as their intuitive conditionals ( $\left.\mathrm{DV}_{15}\right)$, and were taken to examine if and how they would be used by participants in their integration process and posterior calculation. Similar to Surprise (DV 14 ), the measurement of this variable was vulnerable to hindsight bias (Fischhoff \& Beyth-Marom, 1975). Unfortunately, it must be asked after the fact, because to ask it before the results of information search are revealed would bias both search and integration strategies.

These intuitive conditionals were also used to derived Bayesian intuitive posterior $\left(\mathrm{DV}_{15 \mathrm{a}}\right)$, Bayesian matching posterior $\left(\mathrm{DV}_{15 \mathrm{~b}}\right)$, and regression intuitive posterior $\left(\mathrm{DV}_{15 \mathrm{c}}\right)$. These three dependent variables represent posterior probabilities that would be produced using integration strategies that take into account the intuitive conditionals in various ways. Because intuitive conditionals could be used in an infinite number of ways, in order to simplify the analysis, the number of possible 
participant strategies for which combination of intuitive conditionals that they could use was limited to three levels.

First, participants may not use intuitive conditionals at all. (In this case, participants would use only the four selected conditional probabilities, which could form between 0 and 2 pairs. These chosen conditional probabilities were combined using Bayesian integration produce $\mathrm{DV}_{6}$ search phase confirmation bias plus $.50\left(\mathrm{DV}_{6}\right.$ was expressed as the bias from the normative posterior .50). Second, participants may use intuitive conditionals only to complete diagnostic pairs with selected conditional probabilities. (In this case, participants would use the four selected conditional probabilities and between 0 and 4 intuitive conditionals, which could form between 2 and 4 diagnostic pairs). This set of conditional probabilities combined using Bayesian integration produces Bayesian matching posterior $\left(\mathrm{DV}_{15 \mathrm{~b}}\right)$. Third, participants may use intuitive conditionals for every non-selected conditional probability. (In this case, participants would use the four selected conditional probabilities and 12 intuitive conditionals, to form 8 diagnostic pairs). This set of conditional probabilities combined using Bayesian integration produces Bayesian intuitive posterior $\left(\mathrm{DV}_{15 a}\right)$.

Additionally, linear regression was used to produce regression weights for the importance participants placed on the conditional probabilities in each of the four quadrants.

The linear regression was performed on the participants who had all 16 probabilities (the four selected and all twelve of the intuitive conditional probabilities) and predicted the participants' actual posteriors. The conditional probabilities (chosen 
and intuitive were treated as equivalent) were averaged in each quadrant (to reduce the effects of random fluctuations within each quadrant). The four quadrant averages were used to predict the participants' actual posteriors. The resulting regression weights were then applied to each participant’s average conditional probability in each quadrant to produce regression intuitive posterior $\left(\mathrm{DV}_{15 \mathrm{c}}\right)$. This represents the posterior produces given the participants’ chosen and intuitive conditional probabilities and combined using the regression weights.

Because regression is applied across participants, while Bayes’ Theorem is applied by subject, regression requires that all participants have the same information (while Bayes’ Theorem does not). This requirement is met when all 16 selected and intuitive conditionals are used, but is not met with the other two sets of intuitive probabilities. This prevents similar regression dependent variables from being created using only chosen conditional probabilities or for chosen conditional probabilities and matching intuitive conditionals.

\section{Procedure}

Recruitment and experimental setting. Student participants were recruited from psychology classed and at that time they either signed up for appointments or did the experiment in class. Student participants who came to appointments were run in groups that ranged from 1 to approximately 20, with the majority of participants run in groups of approximately 8. Participants who performed the experiment in class were run in a group of 69. The procedure for both the in-class and the appointment subjects was the same, and no differences were detected between administration settings. 
Instructions to the participants. Participants initially received brief oral instructions as follows (Appendix B): to read and sign the informed consent form (Appendix D), what to do to receive extra credit in their psychology class, to remain silent, not to look at their neighbors' papers, and to raise their hands if they had any questions or problems. No participant chose to withdraw from the experiment at his time.

The task itself took approximately 20 minutes to complete. Participants were told to raise their hands when done, at which time they were told individually that they could leave.

Assignment to conditions. Once they had completed the informed consent form they were given one of sixteen scripts (Appendix A) that varied according to the respective levels on the four independent variables (attention, motivation, timing of feedback, size-of-conditionals). The sixteen scripts were each assigned a random number. The rank order of these random numbers determined the order in which each set of 16 scripts was assigned to 16 participants. This process was repeated until all participants were assigned a script.

Experimental control: double blind. This study utilized a double-blind design; both the participant and the experimenter were unaware of which condition the participant received. The only exception to this was in the rare cases of a participant question that required the experimenter to read their script in order to answer their question. 


\section{Materials}

There were 16 scripts, one for each $(2 * 2 * 2 * 2=16)$ experimental condition. All the scripts present the participants with the goal of determining the island of origin of an urn that has been found in the sea between two islands. The cover story was similar to that in Doherty's groundbreaking work (Doherty et al., 1979). The names of the two islands were not counterbalanced, because Doherty et al. (1979) found that counterbalancing had no effect.

The story presented participants with a probability revision problem, asking them to estimate, after they have learned four relevant conditional probabilities, the probability that the urn had been created on one of the islands rather than the other (Table 23 presents the independent variables and their levels. Table 8 presents the types of conditional probabilities participants can select.)

Table 23

Levels of the Four Independent Variables

\begin{tabular}{lcc}
\hline Independent Variables & $\begin{array}{c}\text { Level 1 (Control: } \\
\text { Expected to decrease } \\
\text { biases) }\end{array}$ & $\begin{array}{c}\text { Level 2 (Treatment: } \\
\text { Expected to increase } \\
\end{array}$ \\
\hline $\mathrm{IV}_{1}$ Attention & Balanced & biases) \\
$\mathrm{IV}_{2}$ Motivation & Not Motivated & Motivated \\
$\mathrm{IV}_{3}$ Timing-of-feedback & Sequential & Simultaneous \\
$\mathrm{IV}_{4}$ Size-of-conditionals & Low $p(\mathrm{D} \mid \mathrm{H})$ & High $p(\mathrm{D} \mid \mathrm{H})$ \\
\hline
\end{tabular}


Decision problem introduction. The first two pages of the script included the beginning of the cover story, the hints about the direction and diagnosticy of the conditional probabilities, and the manipulation of the first two independent variables, attention and motivation.

The attention and motivation manipulations structure. The attention (A) manipulation consisted of three parts and the motivation (M) manipulation consisted of two parts. These five parts occur in an AMAMA order. (See IV Operations definitions for more details of these sections.) (Table 24 presents the order of the five parts of these two manipulations, not the full factorial design.) 
Method 126

Table 24

Order of Parts of Manipulation of Attention and Motivation Independent Variables

\begin{tabular}{|c|c|c|c|}
\hline Order & $\begin{array}{l}\text { Independent } \\
\text { Variables }\end{array}$ & Level 1 (control) & Level 2 (treatment) \\
\hline 1 & $\mathrm{IV}_{1}$ Attention & $\begin{array}{l}\text { Both hypotheses equal } \\
\text { and base rate provided }\end{array}$ & Alternate not mentioned \\
\hline 2 & $\begin{array}{l}\mathrm{IV}_{2} \\
\text { Motivation }\end{array}$ & No motivation & Career motivation \\
\hline 3 & $\mathrm{IV}_{1}$ Attention & $\begin{array}{l}\text { Table, all hints, data, } \\
\text { and manipulation } \\
\text { check that asks for the } \\
\text { probability for both } \\
\text { islands }\end{array}$ & $\begin{array}{l}\text { Sequential presentation, hints } \\
\text { favoring focal island, data, and } \\
\text { manipulation check that asks } \\
\text { for the probability for focal } \\
\text { island }\end{array}$ \\
\hline 4 & $\begin{array}{l}\mathrm{IV}_{2} \\
\text { Motivation }\end{array}$ & Nothing & Manipulation Check \\
\hline 5 & $\mathrm{IV}_{1}$ Attention & Nothing & $\begin{array}{l}\text { Hints favoring alternative and } \\
\text { base rate }\end{array}$ \\
\hline
\end{tabular}

The attention manipulation. The attention $\left(\mathrm{IV}_{1}\right)$ manipulation consistent primarily of delaying information about Coral Island. The material was the same for all conditions, though presented in different orders and forms. This material included: the information that the urn had high content in each of eight minerals, hints stating 
the island whose current clay pits are characterized by an abundance of each of these eight minerals, and a paragraph calling into question the value of these hints. (See $\mathrm{IV}_{1}$ Attention operational definition for more details and script quotes.)

The motivation manipulation. The Motivation $\left(\mathrm{IV}_{2}\right)$ manipulation consisted primarily of changes in the second paragraph of the first page of the script. The not motivated condition used island-neutral language, while in contrast, the motivated condition consists of the additional mention of a career incentive to determine that the urn came from Shell Island and a manipulation check and reinforcement question later. (See $\mathrm{IV}_{2}$ Motivation operational definition for more details and script quotes.)

Use of frequency form of data. The base rate information and the later conditional probability information are presented in the form of frequencies instead of probabilities, because Gigerenzer and Hoffrage (1995) found that people understand and use frequency information better.

Direction and diagnosticity of hints. The second half of the first page introduced eight dimensions of potentially diagnostic information (the eight minerals found to be in abundance in the Urn), and a hint for each dimension as to the island that that dimension would favor (specifically, the island whose current clay pits are characterized by an abundance of this mineral). The hints were deliberately ambiguous as to their strength as evidence and as to whether the "evidence" was diagnostic or pseudodiagnostic. Their purpose was to provide a basis for biased information search without introducing any integration bias when using a Bayesian strategy. The hints were balanced, four favoring each island. Because of this balance, they would have no 
effect on a Bayesian probability revision at this point. Additionally, these hints provide pseudodiagnostic participants with a basis for expectations regarding the nonchosen conditional probability, by providing them a basis for an expected value for the diagnostically complementary conditional probability (“intuitive conditionals”) (Alloy \& Tabachnik, 1984). (The participant assumes the probability conditioned on the island the hint favors is greater than the probability conditioned on the island the hint does not favor).

However, for non-Bayesians, balanced information has been found to have a dilution effect in integration, the presence of non-diagnostic information having been found to diminish the impact of diagnostic information (Nisbett, Zucker \& Lemley, 1981; Troutman \& Shanteau 1977). In this case, the dilution effect would bias the participants against confirmation of their hypothesis, and would thus bias the experiment against several of the experimenter's hypotheses.

Information search phase structure. The third page of the script presented the information search phase. On the third page all participants received the same cover story indicating that they could learn any four out of the 16 conditional probabilities. Each conditional probability was specific to one of the two hypotheses (islands) and to one of the eight minerals. Each mineral had been hinted in page one of the scripts as favoring Shell Island or favoring Coral Island. Participants numbered their top four requests for conditional probabilities and then removed opaque stickers to reveal the probabilities they had chosen. 
Method 129

The change to four out of 16 conditional probabilities from the Doherty, et al. (1979) original study, which used six out of 12, was deliberate. The number of conditional probabilities chosen had to be a multiple of four in order to provide participants the opportunity to choose optimally (two matching pairs, with one pair in each diagnostic direction, as inferred from the hints). (This will be demonstrated to be the optimal search strategy later, in the section on Demonstration of the Optimal Search and Integration Strategies). Additionally, the number of possible conditional probabilities had to be four times the number chosen so that participants had the ability to choose all four of their conditional probabilities from any one of the four categories created by the following two independent dimensions: a) probabilities conditioned on the focal or alternate island, and b) probabilities about minerals favoring the focal or alternate island according to the hints.

The first paragraph on the third page of the script suggested that participants pursue, "the most efficient way to determine the origin”, in order to encourage the thought that some of this information was not relevant or less relevant. At this point the last two independent variables were manipulated.

Timing-of-feedback manipulation. The second paragraph on the third page created the third independent variable (timing-of-feedback). In the simultaneous condition, participants were instructed to choose their conditional probabilities all at once before receiving feedback. In the sequential condition, participants were instructed to choose their conditional probabilities one at a time with feedback after each selection, thus enabling them to choose their later selections on the basis of the 
Method 130

results of earlier choices. (See $\mathrm{IV}_{3}$ Timing-of-feedback operational definition for more details and script quotes.)

Size-of-conditionals manipulation. All participants received the feedback (the size of the conditional probabilities) that they had requested as described above (under $\mathrm{IV}_{3}$ : timing-of-feedback). The size of the conditional probabilities was the fourth independent variable. (See $\mathrm{IV}_{4}$ Size-of-conditionals operational definition for more details and script quotes.)

Information search data collection. In the middle of the third page of the script participants were provided with 16 conditional probabilities from which to choose four. (As described in $\mathrm{IV}_{3}$ Timing-of-feedback operational definition for more details and script quotes.) Three search phase dependent variables $\mathrm{DV}_{1}$ : hypothesisfocus bias, $\mathrm{DV}_{2}$ : predictor-selection bias and $\mathrm{DV}_{3}$ : pairs selected were derived from participants' responses to: “Choose 4 of the following 16 tests to perform, as the most efficient way to determine the origin of the Urn of Zor, following the instructions below.”

Integration phase. At the bottom of the third page participants were asked three questions designed to elicit their revised probability that the Urn came from Shell Island and determine the size of their confirmation bias:

Which Island do you now believe the Urn of Zor is more likely to have come from? What do you now believe is the probability that the Urn of Zor came from that island? What do you believe is the probability that the Urn of Zor came from the other island?” 
Method 131

\section{Post hoc questions.}

Information usage dependent variables. The fourth page asked participants what information they used to make their revised probability estimates. They were asked in closed ended format: "What information did you use in making this estimate of the probability of the island of origin? Put a check on the right next to all the pieces of information below that you used.”

Participants were then presented with a list of possibilities that included the base rate, the hints, and the particular conditional probabilities that they had selected. Additionally, a blank space was provided, in case a participant used any additional information not presented in closed-ended format. The responses to the closed-ended questions were used directly to create four dependent variables $\left(\mathrm{DV}_{7}-\mathrm{DV}_{10}\right)$, and indirectly to assist in the classification of participants' integration strategies. (See Classification of Participants Integration Strategies).

Questions for inferring integration strategy. The fifth page asked the following questions designed to help classify participants on the basis of their integration strategies (See discussion of classification of integration strategies in Operational definition of $\mathrm{DV}_{11}$ : integration strategy).

1) Tell me how you went about making your estimate of the probability that the Urn came from the island you chose. You can use a verbal description or a formula to describe the method you used.

2) Place a check next to all the mathematical processes you used? (Check all that apply) 
Method 132

Type of Method Mathematical Example using two pieces of evidence

_ Addition $\quad$ (evidence \#1 + evidence \#2)

_ Subtraction (evidence \#1 - evidence \#2)

_ Multiplication (evidence \#1 * evidence \#2)

_ Division (evidence \#1 / evidence \#2)

Averaging (evidence \#1 + evidence \#2)/ 2

Other

Surprise questions. The fifth page asked the following closed-ended questions designed to determine whether participants were surprised by the values of the conditional probabilities:

3) Did you find the results of any of the tests on the old urns surprising? (Yes / No)

4) If yes, indicate which ones and whether they were higher or lower than expected. (Please look back to see your four selections and then circle higher or lower below).

$\begin{array}{lcc}\text { First Choice Surprising } & \text { higher } & \text { lower } \\ \text { Second Choice Surprising } & \text { higher } & \text { lower } \\ \text { Third Choice Surprising } & \text { higher } & \text { lower } \\ \text { Fourth Choice Surprising } & \text { higher } & \text { lower }\end{array}$

These questions were designed to explore the effects of expectations on search and integration. 
Intuitive conditionals question. The fifth question on page 5 asked participants to: "Please go back to page three again and, without removing any more stickers, write down on the stickers what you think the value of the tests would be that you did not choose to perform.”

Demographic variables. Four demographic questions were asked on page 6 of the script (Race, Gender, Number of College Statistics Classes Taken and G.P.A.). There are no hypotheses for these variables but they will be explored on a post hoc basis, and for the purpose of describing the sample. 
Results 134

\section{Results}

\section{Preliminary Analyses}

Before proceeding with the testing of research and exploratory hypothesis, several preliminary analyses had to be conducted. These include examination of the demographics, manipulation checks, participants’ coherence in their posterior probability estimates, the adequacy of the random assignment, and the nature of the interaction effect between the independent variables attention and motivation. In addition, the results of the classification of participants' search and integration strategies were examined, since this information is required for the analysis of one of the research hypotheses.

Demographics. In order to determine how to proceed with the analysis, the data were examined for demographic effects (school of participant, gender, race, GPA, and number of college statistics classes taken). Since no significant zero order correlations were found between any of the demographic variables and any of the dependent variables, the data were collapsed across the demographic variables.

Manipulation checks. Participants who failed the manipulation checks, in the motivated condition of the motivation IV $(n=13)$ and the balanced condition of the attention IV $(n=12)$ were not dropped. They were not dropped because not all participants received the same number of manipulation checks. Participants whose complementary posterior probabilities failed to sum to one $(n=23)$ were also not dropped because failure of additivity has been found in many situations. Their 
responses were rescaled to sum to one. The rescaling was examined in post hoc tests and found to have no impact on the results.

Coherence checks. Participants’ complementary posterior probabilities (the probability the urn came from Shell and the probability the urn came from Coral) that failed to sum to one $(23 / 324=7.1 \%)$ were rescaled to sum to one. These participants were not dropped, because lack of coherence in posterior probability judgments has been found in many situations. The rescaling was examined in post hoc tests and found to have no impact on the results.

Problem with random assignment. In the simultaneous conditions, there was no possibility of size-of-conditionals affecting search phase selections, since the conditionals were not presented until after the search-phase selections had been made.

Nevertheless, significant differences were found between the two levels of size-of-conditionals for simultaneous participants' use of motivated search strategies, $t(143)=2.12, p=.04, d=.35$. Because search-phase selections preceded the receipt of size-of-conditionals information, such a difference cannot be causal and can be attributed only to a problem with random assignment. For this reason, all participants in the simultaneous conditions were dropped. This prevents examination of the timing of feedback independent variable (since the remaining subjects are all in the sequential feedback condition).

Over-biasing. This study predicted a specific monotonic interaction (subadditive) between the attention and motivation manipulations, whereby focal condition and the motivated condition would not be additive (non-additive effect). Specifically, 
Results 136

it was expected that either biasing condition would be sufficient to cause bias and that the combinations of biasing conditions would result in no more and no less bias than either biasing condition alone (see Expected Effects of Independent Variables for a discussion).

However, for some dependent variables, non-monotonic interactions (often crossover effects) were found (called here an over-biasing effect). Specifically this over-biasing effect was that participants who received either biasing condition alone demonstrated bias, but participant who received both biasing conditions demonstrated less bias or no bias. This result is similar to research conducted by Gaeth and Shanteau (1984) where drawing attention to the biasing information reduce the effect of the bias. Therefore, all analyses involving attention and motivation were modified to examine the over-biasing interaction as three post-hoc orthogonal $t$-tests whenever the interaction between attention and motivation or either main effect was significant or nearly significant. These $t$-tests compared the control condition (balanced and not motivated) against the other three conditions (balanced and motivated, focus and not motivated, focus and motivated), in order to determine whether the effects of attention and motivation were additive, non-additive (as originally hypotheses) or over-biasing.

Search and integration strategy classification results. All participants’ search and integration strategies were classified into categories (as described in $\mathrm{DV}_{4}$ : search strategy and $\mathrm{DV}_{11}$ : integration strategy). A large number of participants chose at least one pair (115 out of 324 participants) in the search phase. Similarly, a large number of participants used a Bayesian integration strategy (88 participants) or the ratio of 
conditionals strategy which is the most similar non-Bayesian strategy (35

participants). Unfortunately many of the non-Bayesian participants ended up classified as default search (53 participants) or integration strategy (100 participants). This clearly demonstrates how hard it is understand and classify non-Bayesian participants’ processes (See Tables 25 and 26). 
Table 25

Frequency of Search Strategies

\begin{tabular}{llc}
\hline Strategy Class & Strategy & $n$ \\
\hline Bayesian Strategies & Bayesian Unbiased & 84 \\
& Bayesian Confirmatory Predictor Bias & 15 \\
& Bayesian Disconfirmatory Predictor Bias & 6 \\
\hline Non-Bayesian One- & Shell Island both Focal and Motivated & 21 \\
Category Strategies & Shell Island Focal and Coral Island Motivated & 6 \\
& Coral Island Focal and Shell Island Motivated & 5 \\
\hline Non-Bayesian Two- & Coral Island both Focal and Motivated & 1 \\
\hline Category Strategies & Coral Island Column Focal & 30 \\
\hline & Shell Island Row Motivated & 0 \\
\hline & Coral Island Row Motivated & 53 \\
& Consistent Diagonal & 0
\end{tabular}


Table 26

Frequency of Integration Strategies

\begin{tabular}{|c|c|c|}
\hline Strategy Class & Strategy & $n$ (Shell / Coral) * \\
\hline \multirow[t]{5}{*}{ Mathematical 2-Island } & Biased Bayesian & $32(18 / 14)$ \\
\hline & Bayesian & 56 \\
\hline & Ratio of Conditionals & 35 \\
\hline & Favoring Ratio & 3 \\
\hline & Double Ratio & 1 \\
\hline \multirow[t]{6}{*}{ Mathematical 1-Island } & Sum of One-Island's Conditionals & $9(5 / 4)$ \\
\hline & Sum of One-Island's Frequencies & $1(0 / 1)$ \\
\hline & Average of One-Island's & $13(8 / 5)$ \\
\hline & Conditionals & \\
\hline & Extreme Conditional & $12(8 / 4)$ \\
\hline & Base Rate + Largest Conditional & $1(1 / 0)$ \\
\hline Mathematical 0-Island & Base Rate & 6 \\
\hline \multirow[t]{5}{*}{ Non-Mathematical } & Non-Mathematical One-Island & $55(44 / 11)$ \\
\hline & Non-Diagnostic Search with & 67 \\
\hline & Unknown Integration & \\
\hline & Diagnostic Search with Unknown & 33 \\
\hline & Integration & \\
\hline
\end{tabular}


Results 140

* Note: Seven strategies were defined by whether the information used was focused on Shell or Coral. For these seven strategies the sample was broken down into the number of participants classified as the Shell or Coral version of that strategy.

\section{Research Hypotheses}

Overall testing strategy for research hypotheses. The experimental research hypotheses were tested by independent sample $t$-tests comparing the participants in biasing vs. non-biasing conditions. The research hypotheses were tested using onetailed $t$-tests, because they were directional hypotheses consistent with prior research. These $t$-test were followed up with three-way ANOVAs (Attention * Motivation * Size-of-Conditionals), to test for any interactions among the three remaining independent variables, (the timing of feedback independent variable was lost to selection effects). Because many dependent variables in this study did not satisfy parametric assumptions (especially, normality), the Mann-Whitney $U$-test was also performed as a backup. However, the results of the Mann-Whitney $U$-tests did not differ from parametric tests. The process for the exploratory experimental hypotheses will be the same as described above except that the initial $t$-tests were omitted, because of the exploratory nature of the hypotheses.

Bonferroni adjustment. In order to provide Bonferroni control for the experiment-wide .05 alpha level, this alpha level was divided into four .0125 alpha levels for the four research hypotheses. For the exploratory hypotheses and post hoc tests, no Bonferroni adjustment was made, and therefore the conclusions drawn from such tests should be considered preliminary. For all these other tests a .05 alpha level 
was set. In the tables that follow, significant and nearly significant findings are indicated by the following symbols: ${ }^{* * *} \mathrm{p}<.001,{ }^{* *} \mathrm{p}<.01,{ }^{*} \mathrm{p}<.05, \wedge \mathrm{p}<.10$.

Research hypotheses results. The research hypotheses are tabulated in Table 27. (Note: Table 27 differs from Table 9, in adding the results for each of the hypotheses, and the simplification of the design because of the selection differences.) The hypotheses are listed by number, in the row and column corresponding to the IV and DV involved. The choice of these hypotheses has been described in the methods section, the descriptions of each hypothesis and the results of the tests follow. 
Table 27

Research Hypotheses Results: Independent and Dependent Variables Involved

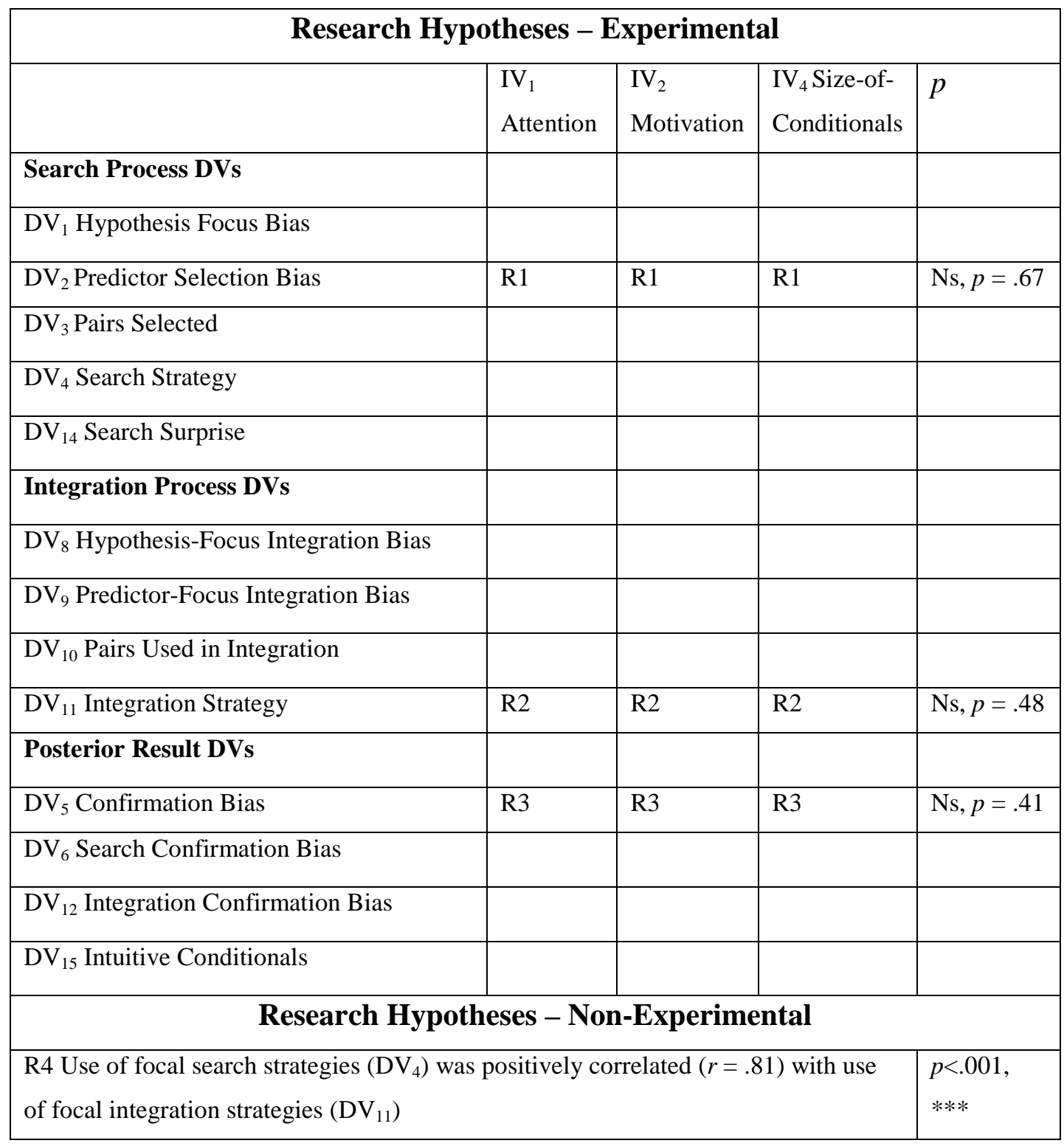

Note: R refers to research hypotheses for that IV DV combination. Due to selection difference participants in the simultaneous conditions were dropped. This resulted in dropping $\operatorname{IV}_{3}$ and the $\mathrm{IV}_{3}$ * $\mathrm{IV}_{4}$ Interaction. The hypothesized unbiasing effects of this interaction are now tested as a main effect of $\mathrm{IV}_{4}$. Many empty cells will be examined by exploratory hypotheses.

$(* * * p<.001, * * p<.01, * p<.05, \wedge p<.10)$ 
Predictor selection bias. Research Hypothesis \#1 was that participants in biased conditions would select more conditional probabilities from minerals that are hinted to favor Shell Island than from minerals hinted to favor Coral Island than would participants in unbiased conditions.

The difference between conditions can be seen as the effect of the biasing conditions of the independent variables. This difference was assessed using an independent samples $t$-test. The results indicates that the biased conditions participants' mean number of conditional probabilities selected about minerals that are hinted to favor Shell Island $(M=2.17, S D=.75)$ were not significantly different than the participants in unbiased conditions $(M=2.30, S D=.89), t(158)=.93, p=.67$. The standardized effect size index was $d=.15$, indicating a small difference in the opposite direction from that expected.

The follow-up three-way ANOVA (Attention * Motivation * Size-ofConditionals) was not significant, $F(7,152)=.38, p=.91$, partial $\eta^{2}=.02$. Inconsistent with Research Hypothesis \#1 all three main effects and the Attention * Motivation interaction were non-significant (see Table 28). Interestingly, the planned curative low conditionals $(M=2.33, S D=.91)$ instead non-significantly increased bias compared to high conditionals $(M=2.16, S D=.76)$. 
Table 28

The Effects of the Independent Variables on Predictor Selection Bias

\begin{tabular}{llll}
\hline Effect & $F(1,152)$ & $P$ & partial $\eta^{2}$ \\
\hline Attention & .05 & .83 & $<.01$ \\
Motivation & $<.01$ & .96 & $<.01$ \\
Size-of-Conditionals & 1.62 & .21 & $=.01$ \\
Attention*Motivation & .20 & .65 & $<.01$ \\
\hline
\end{tabular}

Biased Bayesian strategy in integration. In order to avoid non-independence of hypotheses, Hypotheses \#2 and \#3 were tested on different participants. Research Hypothesis \#2 was that participants in biasing conditions who searched optimally (chose two pairs, with one pair expected to favor each island) would be more likely in integration to use the selected pair favoring shell than the pair favoring coral (Biased Bayesian Integration Strategy) than would participants in the non-biasing conditions. The difference between conditions can be seen as the effect of the biasing conditions of the independent variables. This difference was assessed using an independent samples $t$-test. The results indicates that participants in biased conditions were more likely to use the biased Bayesian strategy favoring Shell Island $(M=.21, S D=.59)$, but not significantly more than the participants in unbiased conditions $(M=.20, S D=$ $.41), t(37)=.05, p=.48$. The standardized effect size index was $d=.02$, indicating a very small effect that was in the expected direction. 
The follow-up three-way ANOVA (Attention * Motivation * Size-ofConditionals) was not significant, $F(7,31)=1.87, p=.11$, partial $\eta^{2}=.30$. Inconsistent with Research Hypothesis \#2, all three main effects and the Attention * Motivation Interaction were non-significant (see Table 28).

Table 29

The Effects of the Independent Variables on Biased Bayesian Strategy

\begin{tabular}{llll}
\hline Effect & $F(1,31)$ & $P$ & partial $\eta^{2}$ \\
\hline Attention & .04 & .84 & $<.01$ \\
Motivation & .46 & .50 & $=.02$ \\
Size-of-Conditionals & .68 & .41 & $=.02$ \\
Attention*Motivation & 3.58 & .07 & $=.10$ \\
Attention*Size & 3.04 & .09 & $=.09$ \\
\hline
\end{tabular}

However, two two-way interactions were nearly significant: Attention* Motivation, and Attention* Size-of-Conditionals. The first interaction was the overbiasing effect discussed earlier and was explored by the three post hoc orthogonal $t$ tests (see Figure 3). The three post-hoc comparisons were all non-significant but the simple main effect of motivation when attention was balanced was nearly significant (with a very small sample size and a large effect size, see Table 30). The second interaction was low conditionals causing bias towards the focal hypothesis when people were not focused (see Figure 4). 
Results 146

Table 30

Post Hoc Orthogonal Contrasts Exploring Over-Biasing Effect on use of Biased Bayesian Strategies

\begin{tabular}{llll}
\hline Contrast Balanced and Not & $t$ & $P$ & $d$ \\
Motivated to: & & & \\
\hline Balanced and motivated & $t(16)=1.60$ & $=.13$ & .90 \\
Focused and not motivated & $t(21)=.97$ & $=.34$ & .72 \\
Focused and motivated & $t(14)=.18$ & $=.86$ & .50 \\
\end{tabular}

Figure 3. Attention by motivation interaction on use of biased Bayesian integration strategies.

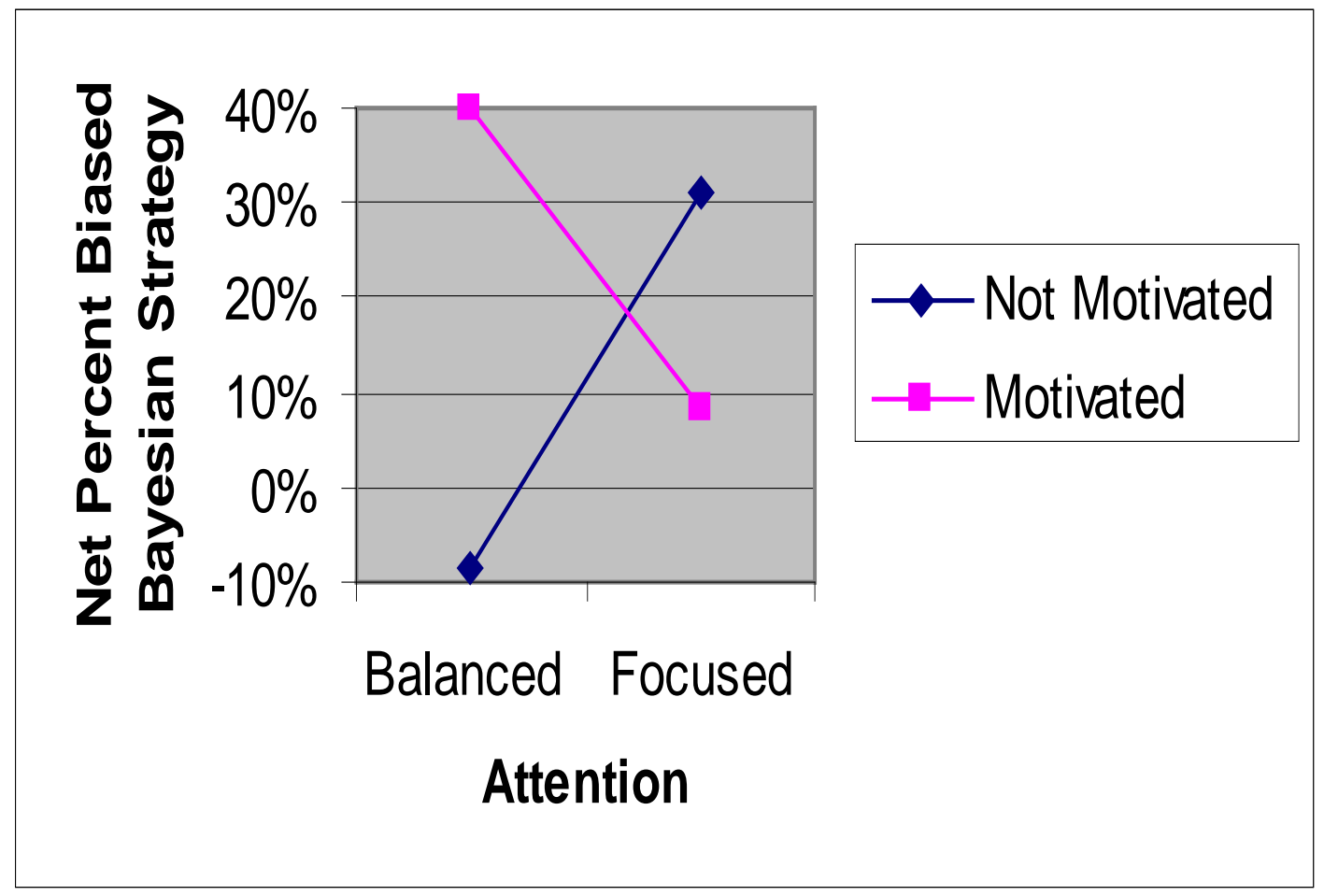


Figure 4. Attention by size-of-conditionals interaction on use of biased Bayesian integration strategies.

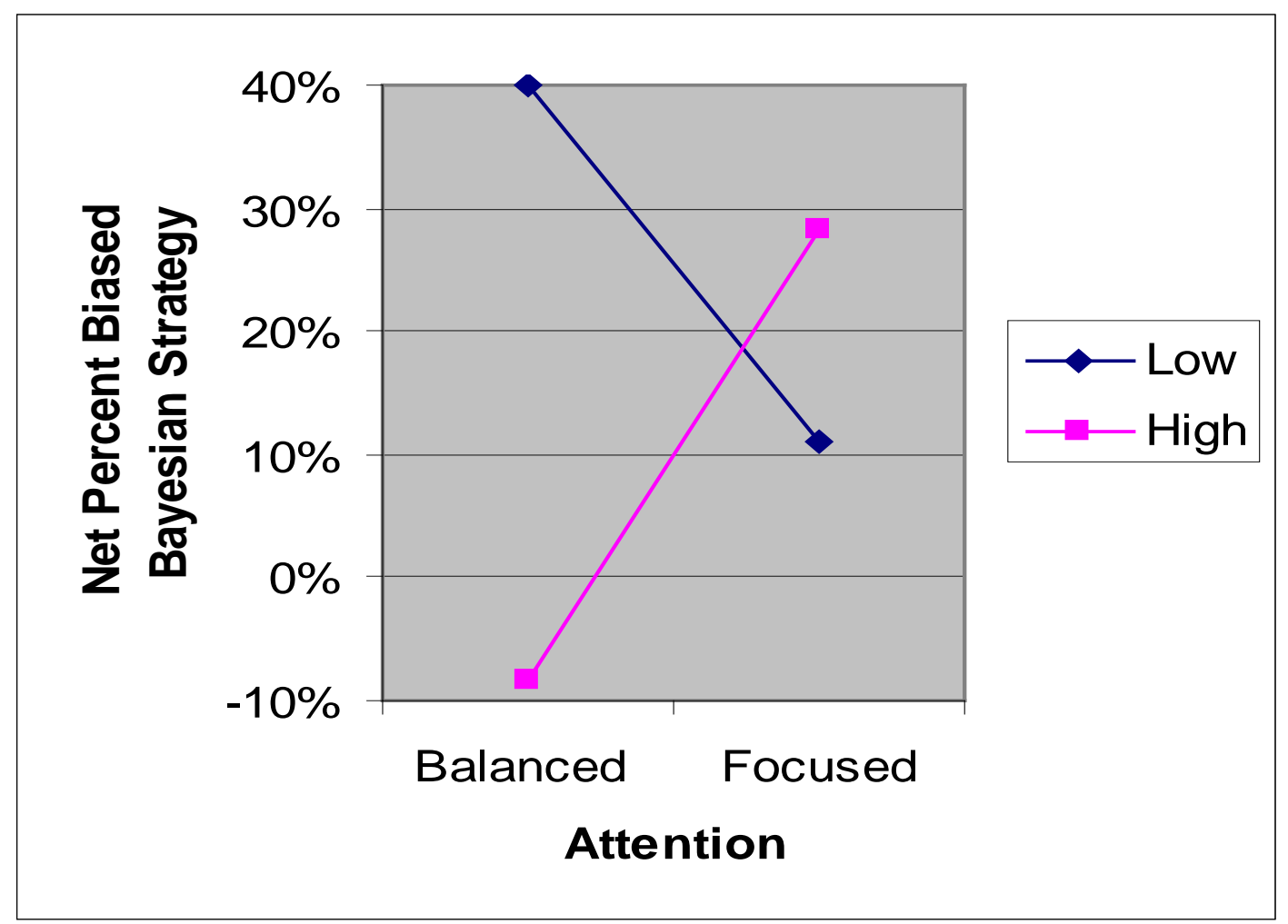

Confirmation bias. In order to avoid non-independence of hypotheses,

Hypotheses 2 and 3 were tested on different participants. Research Hypothesis \#3 was that participants in biasing conditions who did not search optimally (chose two pairs, with one pair expected to favor each island) would produce posterior probabilities more in favor of Shell Island than Coral Island than would participants in unbiased conditions. The difference between conditions can be seen as the effect of the biasing conditions of the independent variables. This difference was assessed using an independent samples $t$-test. 
The results indicates that participants in biased conditions were more likely to use the biased Bayesian strategy favoring Shell Island $(M=5.87, S D=16.34)$ but this was not significantly more than the participants in unbiased conditions $(M=3.96, S D$ $=19.36), t(119)=.58, p=.28$. The standardized effect size index was $d=.10$, indicating a small effect which was in the expected direction.

The follow-up three-way ANOVA (Attention * Motivation * Size-ofConditionals) was not significant, $F(7,113)=.54, p=.81$, partial $\eta^{2}=.03$. Inconsistent with Research Hypothesis \#3, all three main effects and the Attention * Motivation Interaction were non-significant (see Table 31).

Table 31

The Effects of the Independent Variables on Confirmation Bias

\begin{tabular}{lccc}
\hline Effect & $F(1,31)$ & $P$ & partial $\eta^{2}$ \\
\hline Attention & .15 & .70 & $<.01$ \\
Motivation & .81 & .37 & $=.01$ \\
Size-of-Conditionals & .55 & .46 & $=.01$ \\
Attention*Motivation & 1.22 & .27 & $=.01$ \\
\hline
\end{tabular}

Non-independence of search and integration strategies. Research Hypothesis \#4 was that participants who use search strategies that are hypothesis focused will be more likely to use integration strategies that are hypothesis focused. Consistent with Research Hypothesis \#4 the Pearson's $r$ coefficient was significant, indicating that the 
use of focal search strategies predicted the use of focal integration strategies, $r(125)=$ .81, $p>$.001. (See Table 32 for cross-tabulation).

Table 32

Cross Tabulation of Search and Integration Strategies by Focal Island

\begin{tabular}{l|lll|l}
\hline & Shell Focused & Balanced & Coral Focused & Total \\
& Search & Search & Search & \\
\hline Shell Focused Integration & 26 & 19 & Not Possible & 45 \\
Balanced Integration & 0 & 103 & 0 & 103 \\
Coral Focused Integration & Not Possible & 9 & 3 & 12 \\
\hline Total & 3 & 131 & 26 & 160 \\
\hline
\end{tabular}

An alternative way to examine this hypothesis would be to exclude the 29 participants who choose four probabilities focused on a single island (because they are restricted from integrating in the opposite focal direction), and instead focus on the other participants who were not prevented from integrating in either focal way. In order to do this, participants who chose either three or one probabilities conditioned on the Shell Island would be treated as Shell focused and Coral focused respectively. (In the prior analysis such participants were dropped since their search strategy was considered unclassifiable because they were not consistent). For these participants, the Pearson's $r$ coefficient was not significant $r(128)=.02, p=.83$. This indicates no 
Results 150

relationship between the focus of search selections and focal integration strategies for participants who did not choose all four probabilities conditioned on the same island.

Table 33

Cross Tabulation of Search and Integration Strategies by Conditionals Selected

\begin{tabular}{|c|c|c|c|c|}
\hline & $\begin{array}{l}3 \text { probabilities } \\
\text { conditioned on } \\
\text { Shell }\end{array}$ & $\begin{array}{l}2 \text { probabilities } \\
\text { conditioned on } \\
\text { Shell }\end{array}$ & $\begin{array}{l}1 \text { probabilities } \\
\text { conditioned on } \\
\text { Shell }\end{array}$ & Total \\
\hline $\begin{array}{l}\text { Shell Focused } \\
\text { Integration }\end{array}$ & 0 & 5 & 1 & 6 \\
\hline $\begin{array}{l}\text { Balanced } \\
\text { Integration }\end{array}$ & 12 & 100 & 3 & 115 \\
\hline $\begin{array}{l}\text { Coral Focused } \\
\text { Integration }\end{array}$ & 0 & 6 & 1 & 7 \\
\hline Total & 12 & 111 & 5 & 128 \\
\hline
\end{tabular}

These analyses indicate that participants who searched for information conditioned exclusively one hypothesis chose to use focal integration strategies despite non-focal ones being available to them (base rate or non-mathematical). However, for participants who are focal but not exclusive (one or three probabilities conditioned on Shell) or participants who use a balanced search strategy their choice of focal or non-focal integration strategies appears random. This supports the idea that 
Results 151

the two phases are primarily (but not completely) independent, because even the participants who used the most biased search strategy (four probabilities conditioned on the same island) utilized different focal integration strategies. For example, among the 16 participants who used the most common Shell focal search strategy (Shell island column focal) and a focal integration strategy, there were four different focal integration strategies employed: Sum of one islands conditionals $(n=1)$, extreme conditional ( $n=2)$, average of one-island conditionals $(n=1)$, and non-mathematical one-island $(n=12)$. These integration strategies produce very different posteriors (in fact the last strategy is a catch-all for participants who mention using conditionals from only one-island but do not specify how they use them), resulting in a wide range of posteriors. The 16 participants had posteriors ranging from $20 \%$ to $100 \%$ ( $M=$ $16.5, S D=22.15)$

\section{Exploratory Hypotheses}

These hypotheses are less central to the study and therefore were not included in the Bonferroni adjustment. Therefore, conclusions drawn from them should be considered preliminary. As with the experimental research hypotheses, the overbiasing effect will be examined any time Attention or Motivation or their interaction is at least nearly significant.

The hypotheses are tabulated below. The hypotheses are listed by number (research hypotheses R1, R2 ... and exploratory hypotheses H1, H2 ...). Significant and nearly significant results appear in the column corresponding to the IV, and in the row corresponding to the DV involved. Descriptions of each exploratory hypothesis 
Results 152

follow. (Note: Table 34 is a slight refinement of Table 10, adding the results of the test and showing the changes described in Exploratory Follow-Up to the Research Hypotheses.)

\section{Table 34}

Exploratory Hypotheses Results: Independent and Dependent Variables Involved

\begin{tabular}{|c|c|c|c|c|c|}
\hline \multicolumn{6}{|c|}{ Exploratory Hypotheses - Experimental } \\
\hline & $\begin{array}{l}\text { Hypo- } \\
\text { theses }\end{array}$ & $\begin{array}{l}\mathrm{IV}_{1} \\
\text { Attention }\end{array}$ & $\begin{array}{l}\mathrm{IV}_{2} \\
\text { Motivation }\end{array}$ & $\begin{array}{l}\mathrm{IV}_{4} \text { Size-of- } \\
\text { Conditionals }\end{array}$ & Post-hoc \\
\hline \multicolumn{6}{|l|}{ Search Process DVs } \\
\hline $\mathrm{DV}_{1}$ Hypothesis Focus Bias & $\begin{array}{l}\text { E1 } \\
\text { E4 }\end{array}$ & $\begin{array}{l}p=.07 \wedge \\
p=.09 \wedge\end{array}$ & $p=.07 \wedge$ & & Additive \\
\hline $\begin{array}{l}\mathrm{DV}_{2} \text { Predictor Selection } \\
\text { Bias }\end{array}$ & $\begin{array}{l}\text { R1 ns } \\
\text { E5 ns }\end{array}$ & & & & \\
\hline $\mathrm{DV}_{3}$ Pairs Selected & E2 ns & & & & \\
\hline $\mathrm{DV}_{4}$ Search Strategy & E3 & $p=.04 *$ & $p=.04 *$ & $p=.05 *$ & Additive \\
\hline $\mathrm{DV}_{14}$ Search Surprise & $\begin{array}{l}\text { E15 ns } \\
\text { E16 ns }\end{array}$ & & & & \\
\hline \multicolumn{6}{|l|}{$\begin{array}{l}\text { Integration Process } \\
\text { DVs }\end{array}$} \\
\hline $\begin{array}{l}\mathrm{DV}_{8} \text { Hypothesis-Focus } \\
\text { Integration Bias }\end{array}$ & E6 ns & & & & \\
\hline $\begin{array}{l}\mathrm{DV}_{9} \text { Predictor-Focus } \\
\text { Integration Bias }\end{array}$ & E7 ns & & & & \\
\hline $\begin{array}{l}\mathrm{DV}_{10} \text { Pairs Used in } \\
\text { Integration }\end{array}$ & E8 ns & & & & \\
\hline $\mathrm{DV}_{11}$ Integration Strategy & $\begin{array}{l}\text { R2 } \\
\text { E9 }\end{array}$ & $\begin{array}{l}p=.09 \wedge \\
p=.08 \wedge\end{array}$ & $p=.07 \wedge$ & & $\begin{array}{l}\text { Overbias } \\
\text { Sub- } \\
\text { Additive }\end{array}$ \\
\hline
\end{tabular}




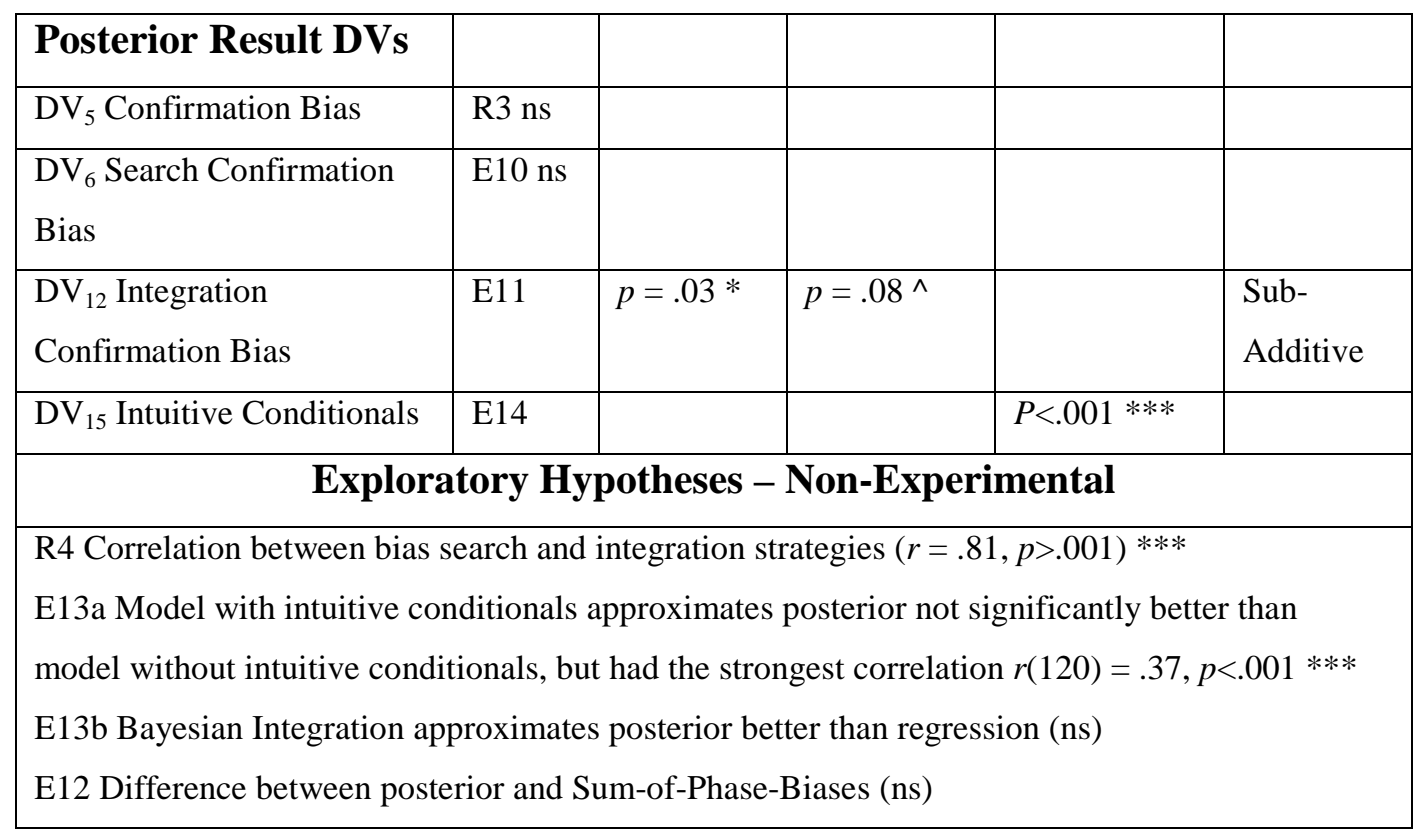

Note: The Rs represent the research hypotheses and Es represent exploratory hypotheses.

$\left({ }^{* * *} \mathrm{p}<.001,{ }^{* *} \mathrm{p}<.01,{ }^{*} \mathrm{p}<.05, \wedge \mathrm{p}<.10\right)$

Exploratory search process hypotheses. All these hypotheses involve dependent variables about participants’ search phase selection decisions.

Hypothesis focus bias. Exploratory Hypothesis \#1, as has been demonstrated elsewhere, was that participants in biasing conditions would select more probabilities conditioned on Shell Island than conditioned on Coral Island than would participants in unbiased conditions. A three-way (Attention * Motivation * Size-of-Conditionals) ANOVA was performed and was not significant, $F(7,152)=1.486, p=.176$, partial $\eta^{2}=.06$. Partially supportive of Exploratory Hypothesis \#1 the main effects of both attention and motivation were nearly significant (see Table 35). 
Table 35

The Effects of the Independent Variables on Hypothesis Focus Bias

\begin{tabular}{llll}
\hline Effect & $F(1,152)$ & $p$ & partial $\eta^{2}$ \\
\hline Attention & 3.35 & .07 & .02 \\
Motivation & 3.41 & .07 & .02 \\
Size-of-Conditionals & .32 & .57 & $<.01$ \\
Attention*Motivation & .01 & .91 & $<.01$ \\
\hline
\end{tabular}

Because attention and motivation were nearly significant, the over-biasing effect was explored by the three post hoc orthogonal $t$-tests. The two simple main effects post-hoc comparisons were both non-significant. In contrast the comparison balanced and not motivated versus focused and motivated was significant. This provides mixed evidence that attention and motivation have weak and additive effects on predictor selection bias (see Tables 36 and 37 and Figure 5).

Table 36

Orthogonal Contrasts on Over-Biasing Effect on Hypothesis Focus Bias

\begin{tabular}{llll}
\hline Contrast Balanced and Not Motivated to: & $t$ & $p$ & $d$ \\
\hline Balanced and motivated & $t(79)=1.33$ & $=.19$ & .29 \\
Focused and not motivated & $t(79)=1.26$ & $=.21$ & .28 \\
Focused and motivated & $t(76)=2.28$ & $=.03$ & .50 \\
& & & \\
\hline
\end{tabular}


Table 37

Descriptive Statistics on Over-Biasing Effect on Hypothesis Focus Bias

\begin{tabular}{llc}
\hline Attention and Motivation Conditions: & $M$ & $S D$ \\
\hline Balanced and not motivated & 2.13 & .91 \\
Balanced and motivated & 2.37 & .70 \\
Focused and not motivated & 2.37 & .80 \\
Focused and motivated & 2.63 & 1.05 \\
\hline
\end{tabular}

Figure 5. Attention by motivation interaction on hypothesis focus bias.

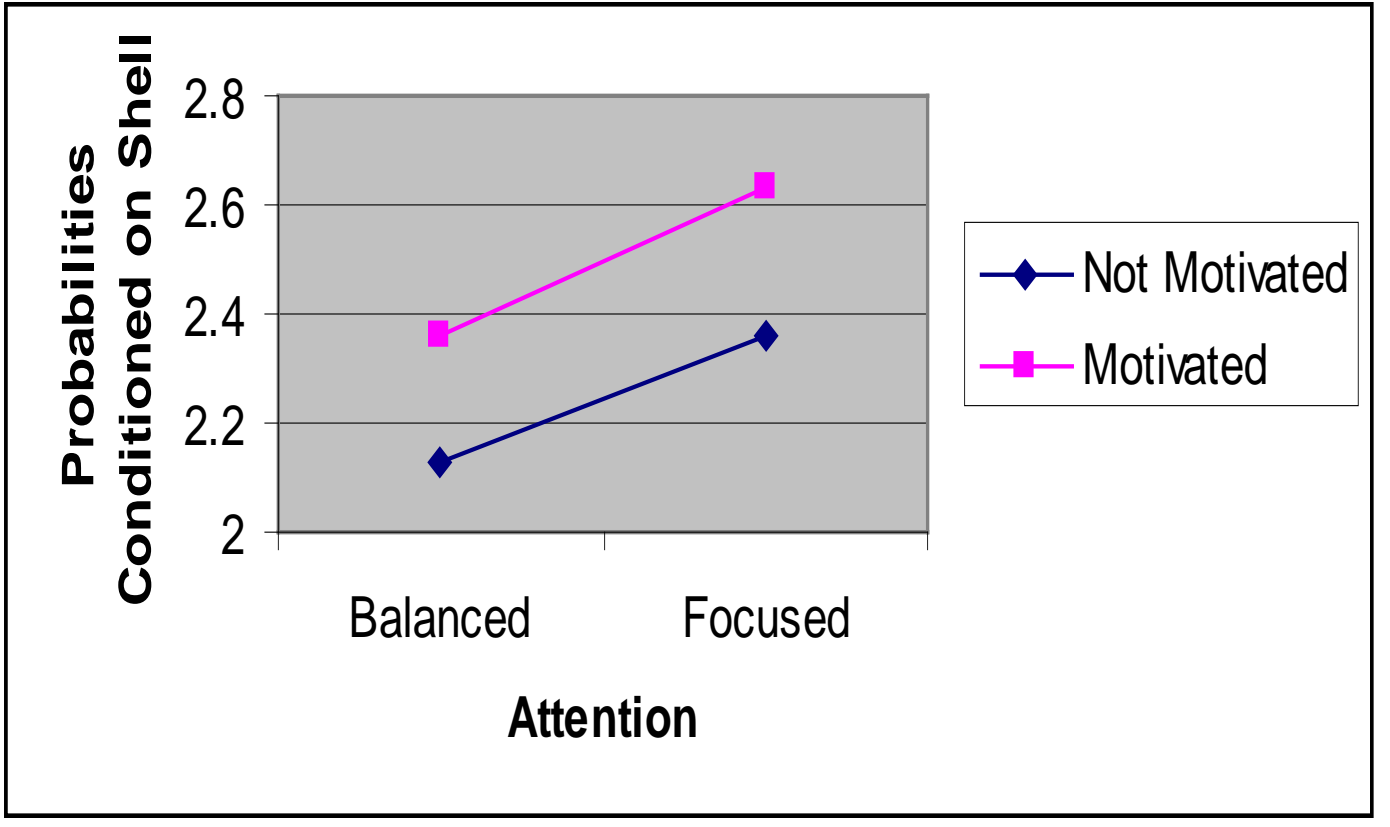

Pseudodiagnosticity. Exploratory Hypothesis \#2, as demonstrated elsewhere, was that participants in biasing conditions would select fewer diagnostic pairs than would participants in unbiased conditions. The three-way (Attention * Motivation * 
Results 156

Size-of-Conditionals) ANOVA was not significant, $F(7,152)=.86, p=.54$ partial $\eta^{2}$ $=.04$. Inconsistent with Exploratory Hypothesis \#2 all main effects and interactions were not significant (see Table 38). In contrast to Doherty et al. (1979), it should be noted that participants performance (.75 pairs selected on average) was statistically $[t(159)=5.03, p<.001]$ better than chance $(.40$ pairs $)$.

Table 38

The Effects of the Independent Variables on Pseudodiagnosticity

\begin{tabular}{lccc}
\hline Effect & $F(1,152)$ & $p$ & partial $\eta^{2}$ \\
\hline Attention & .126 & .72 & $<.01$ \\
Motivation & 1.44 & .23 & .01 \\
Size-of-Conditionals & .66 & .42 & $<.01$ \\
Attention*Motivation & .79 & .37 & $<.01$ \\
\hline
\end{tabular}

Focal search strategies. Exploratory Hypothesis \#3 was that participants in biasing conditions would use more hypothesis-focused search strategies and fewer control focused search strategies than would participants in unbiased conditions. Due to their lack of a consistent search strategy, 35 participants were dropped from this analysis. A three-way (Attention * Motivation * Size-of-Conditionals) ANOVA was performed and was nearly significant, $F(7,117)=1.97, p=.07$ partial $\eta^{2}=.11$ (see Table 39). 
Table 39

The Effects of the Independent Variables on Focal Search Strategies

\begin{tabular}{llll}
\hline Effect & $F(1,152)$ & $p$ & partial $\eta^{2}$ \\
\hline Attention & 4.38 & .04 & .04 \\
Motivation & 4.29 & .04 & .04 \\
Size-of-Conditionals & .70 & .40 & .01 \\
Attention*Motivation & .03 & .70 & $<.01$ \\
Attention*Size & 3.96 & .05 & .03 \\
\hline
\end{tabular}

Consistent with Exploratory Hypothesis \#3, both the attention and motivation variables led to significant differences. Because attention and motivation were significant, the over-biasing effect was explored by three post hoc orthogonal $t$-tests. The two simple main effects post-hoc comparisons were both non-significant. In contrast, the comparison of balanced and not motivated versus focused and motivated was significant. These post hoc tests provide evidence that in the case of predictor selection bias attention and motivation have additive effects. (See Tables 40 and 41 and Figure 6). 
Table 40

Orthogonal Contrasts on Over-Biasing Effect on Focal Search Strategies

\begin{tabular}{llll}
\hline Contrast Balanced and Not Motivated to: & $t$ & $p$ & $d$ \\
\hline Balanced and motivated & $t(79)=1.33$ & $=.25$ & .30 \\
Focused and not motivated & $t(79)=1.26$ & $=.21$ & .32 \\
Focused and motivated & $t(76)=2.41$ & $=.02$ & .59 \\
\hline
\end{tabular}

Table 41

Descriptive Statistics on Over-Biasing Effect on Focal Search Strategies

\begin{tabular}{lcc}
\hline Attention and Motivation Conditions: & $M$ & $S D$ \\
\hline Balanced and not motivated & .04 & .44 \\
Balanced and motivated & .16 & .37 \\
Focused and not motivated & .17 & .38 \\
Focused and motivated & .35 & .55 \\
\hline
\end{tabular}


Figure 6. Attention by motivation interaction on use of focal search strategies.

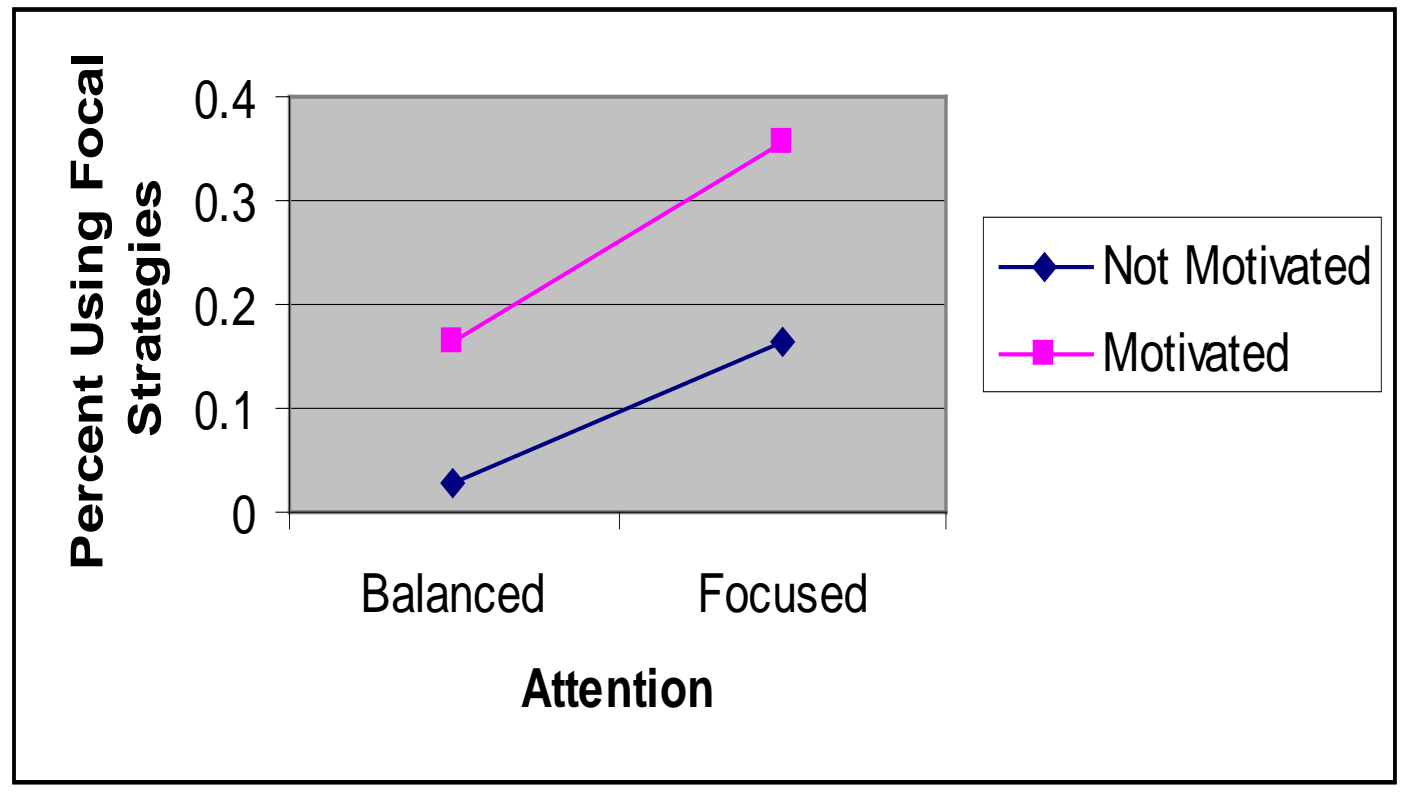

Additionally, the two-way interaction between attention and size-ofconditionals was significant, where the biasing affect of attention only works with low conditional probabilities. This provides further evidence that the expected curative effect of surprise did not work (see Figure 7). 
Figure 7. Attention by size-of-conditionals interaction on use of focal search strategies.

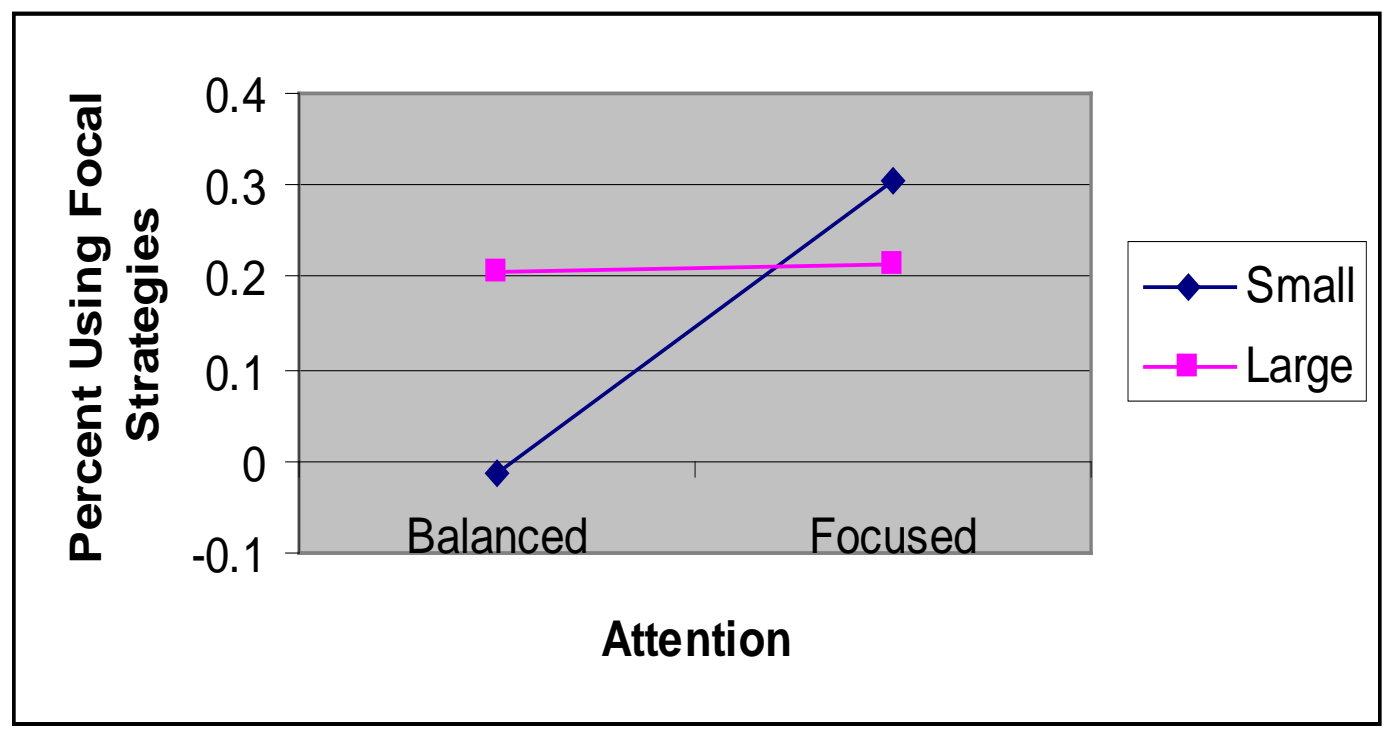

Comparing the size of hypothesis focus and predictor selection biases.

Exploratory Hypotheses \#4 and \#5 compared the effects of attention and motivation, respectively, on hypothesis focus bias and predictor selection bias. This is possible because these two dependent variables are on the same scale $0-4$ selections made on the 8 possible selections favoring one island.

Exploratory Hypothesis \#4 was that the focal condition would have a bigger impact on hypothesis focus bias than on predictor selection bias. This difference was assessed using a paired samples $t$-test. The results indicates that the focused conditions caused greater hypothesis focus bias $(M=2.49, S D=.93)$ than predictor selection bias $(M=2.27, S D=.82)$. This difference was found to be nearly significant, $T(78)=$ 1.706, $p=.09, \mathrm{~d}=.19$.

Exploratory Hypothesis \#5 was that the motivated condition would have a bigger impact on predictor selection bias than on hypothesis focus bias. This 
difference was assessed using a paired samples $t$-test. However, the results indicates that the motivated conditions caused greater hypothesis focus bias $(M=2.49, S D=$ .89) than predictor selection bias $(M=2.25, S D=.82)$. This difference was found to be nearly significant, $T(78)=1.706, p=.09, \mathrm{~d}=.20$.

Exploratory integration process hypotheses. These hypotheses test the participants' use in integration of the information they selected. Because the information available to each participant at this point is different, these results are a reflection of the combination of both the participants' search phase and integration phase. Additionally, because participants do not have to use all the conditional probabilities made available to them, these hypotheses are tested against difference scores (e.g. focal - non-focal).

Hypothesis-focus integration bias. Exploratory Hypothesis \#6 was that participants in biasing conditions would report a greater difference in the usage of information conditioned on Shell Island and Coral Island than would participants in non-biasing conditions. A three-way (Attention * Motivation * Size-of-Conditionals) ANOVA was performed and was not significant, $F(7,152)=.88, p=.52$, partial $\eta^{2}=$ .04. Inconsistent with Exploratory Hypothesis \#6 both attention, motivation and their interaction were not significant (see Table 42). 
Table 42

The Effects of the Independent Variables on Hypothesis-Focus Integration Bias

\begin{tabular}{llll}
\hline Effect & $F(1,152)$ & $p$ & partial $\eta^{2}$ \\
\hline Attention & 1.23 & .27 & .01 \\
Motivation & .41 & .52 & $<.01$ \\
Size-of-Conditionals & .94 & .33 & .01 \\
Attention*Motivation & .91 & .34 & .01 \\
\hline
\end{tabular}

Predictor-focus integration bias. Exploratory Hypothesis \#7 was that participants in biasing conditions would report a greater difference in the usage of information hinted to favor Shell Island and Coral Island than would participants in unbiased conditions. A three-way (Attention * Motivation * Size-of-Conditionals) ANOVA was performed and was not significant, $F(7,152)=.67, p=.70$, partial $\eta^{2}=$ .03. Inconsistent with Exploratory Hypothesis \#7 both attention, motivation and their interaction were not significant (see Table 43). 
Table 43

The Effects of the Independent Variables on Predictor-Focus Integration Bias

\begin{tabular}{llll}
\hline Effect & $F(1,152)$ & $p$ & partial $\eta^{2}$ \\
\hline Attention & .07 & .79 & $<.01$ \\
Motivation & .19 & .66 & $<.01$ \\
Size-of-Conditionals & 1.50 & .22 & .01 \\
Attention*Motivation & .722 & .40 & .01 \\
\hline
\end{tabular}

Pairs used in integration. Exploratory Hypothesis \#8 was that participants in biasing conditions report lesser usage of diagnostic pairs than would participants in unbiased conditions. A three-way (Attention * Motivation * Size-of-Conditionals) ANOVA was performed and was not significant, $F(7,152)=.71, p=.66$, partial $\eta^{2}=$ .03. Inconsistent with Exploratory Hypothesis \#7 both attention, motivation and their interaction were not significant (see Table 44).

Table 44

The Effects of the Independent Variables on Pairs Used in Integration

\begin{tabular}{llll}
\hline Effect & $F(1,152)$ & $p$ & partial $\eta^{2}$ \\
\hline Attention & .02 & .90 & $<.01$ \\
Motivation & .08 & .77 & $<.01$ \\
Size-of-Conditionals & $<.01$ & .98 & $<.01$ \\
Attention*Motivation & .60 & .44 & $<.01$ \\
\hline
\end{tabular}


Use of biased integration strategies. Exploratory Hypothesis \#9 was that participants in biasing conditions would be more likely to be classified as using biased integration strategies than would participants in unbiased conditions. The three-way ANOVA was not significant, $F(7,152)=.92, p=.50$, partial $\eta^{2}=.03$. Inconsistent with Exploratory Hypothesis \#9 attention was nearly significant $[F(1,152)=3.080, p$ $=.081]$, while motivation $[F(1,152)=.836, p=.362]$ and their interaction $[F(1,152)$ $=.935, p=.335]$ were not significant (see Table 45).

Table 45

The Effects of the Independent Variables on Use of Biased Integration Strategies

\begin{tabular}{llll}
\hline Effect & $F(1,152)$ & $p$ & partial $\eta^{2}$ \\
\hline Attention & 3.08 & .08 & .02 \\
Motivation & .84 & .36 & .01 \\
Size-of-Conditionals & .03 & .85 & $<.01$ \\
Attention*Motivation & .94 & .33 & .01 \\
\hline
\end{tabular}

Because attention was nearly significant, the over-biasing effect was explored by the three post hoc orthogonal $t$-tests. The simple main effect comparison for attention was significant while the comparison for motivation was not. The comparison of balanced and not motivated versus focused and motivated was nearly significant. These post hoc tests provide weak evidence that in the case of the use of biased integration strategies attention and motivation have sub-additive effects 
Results 165

(attention and motivation in combination produce approximately the same bias as each alone). (See Tables 46 and 47).

Tables 46

Orthogonal Contrasts on Over-Biasing Effect on Use of Biased Integration Strategies

\begin{tabular}{llll}
\hline Contrast Balanced and Not Motivated to: & $T$ & $p$ & $d$ \\
\hline Balanced and motivated & $t(79)=1.41$ & $=.16$ & .31 \\
Focused and not motivated & $t(79)=2.03$ & $=.05$ & .44 \\
Focused and motivated & $t(76)=1.72$ & $=.09$ & .37 \\
\hline
\end{tabular}

Table 47

Descriptive Statistics on Over-Biasing Effect on Use of Biased Integration Strategies

\begin{tabular}{llc}
\hline Attention and Motivation Conditions: & $M$ & $S D$ \\
\hline Balanced and not motivated & .03 & .58 \\
Balanced and motivated & .20 & .51 \\
Focused and not motivated & .27 & .50 \\
Focused and motivated & .26 & .64 \\
\hline
\end{tabular}

Phase biases. Exploratory Hypotheses \#10 and \#11 examine the effects of the independent variables on the search and integration strategies confirmatory effects (confirmation bias as defined by this study). These effects were measured in terms of 
the bias of the posterior probability that was caused by their biased strategies in each phase individually.

Search confirmation bias. Exploratory Hypothesis \#10 was that participants in biasing conditions would have a higher posterior probability produced from optimal integration and their actual search than would participants in balanced conditions. A three-way (Attention * Motivation * Size-of-Conditionals) ANOVA was performed and was not significant, $F(7,152)=.44, p=.88$ partial $\eta^{2}=.02$. Inconsistent with Exploratory Hypothesis \#10, attention, motivation and their interaction were not significant (see Table 48).

Table 48

The Effects of the Independent Variables on Search Confirmation Bias

\begin{tabular}{llll}
\hline Effect & $F(1,152)$ & $P$ & partial $\eta^{2}$ \\
\hline Attention & 1.28 & .26 & .01 \\
Motivation & .03 & .86 & $<.01$ \\
Size-of-Conditionals & .02 & .88 & $<.01$ \\
Attention*Motivation & 1.11 & .29 & .01 \\
\hline
\end{tabular}

Integration confirmation bias. Exploratory Hypothesis \#11 was that participants in biasing conditions would have a higher posterior probability produced from optimal search and their actual integration strategy than would participants in 
balanced conditions. A three-way (Attention * Motivation * Size-of-Conditionals) ANOVA was performed and was not significant, $F(7,152)=1.51, p=.17$, partial $\eta^{2}=$ .07. The main effect of attention was significant and the interaction of motivation and attention was nearly significant. In contrast, motivation was not significant (see Table 49).

Table 49

The Effects of the Independent Variables on Integration Confirmation Bias

\begin{tabular}{llll}
\hline Effect & $F(1,152)$ & $P$ & ${\text { partial } \eta^{2}}^{2}$ \\
\hline Attention & 4.66 & .03 & .03 \\
Motivation & .69 & .41 & $<.01$ \\
Size-of-Conditionals & .19 & .66 & $<.01$ \\
Attention*Motivation & 3.11 & .08 & .02 \\
\hline
\end{tabular}

Because attention was a significant factor and the Attention * Motivation interaction was nearly significant, the over-biasing effect was explored by three post hoc orthogonal $t$-tests. All three comparisons were significant (balanced and not motivated versus balanced and motivated, balanced and not motivated versus focused and not motivated and balanced and not motivated versus focused and motivated). The relative equality of the effects provides evidence that attention and motivation have sub-additive effects on integration confirmation bias (See Tables 50 and 51 and Figure 8). 
Table 50

Orthogonal Contrasts on Over-Biasing Effect on Integration Confirmation Bias

\begin{tabular}{llll}
\hline Contrast Balanced and Not Motivated to & $T$ & $p$ & $d$ \\
\hline Balanced and motivated & $t(79)=2.06$ & $=.04$ & .45 \\
Focus and not motivated & $t(79)=2.55$ & $=.01$ & .55 \\
Focus and motivated & $t(76)=2.26$ & $=.03$ & .50 \\
\hline
\end{tabular}

Table 51

Descriptive Statistics on Over-Biasing Effect on Integration Confirmation Bias

\begin{tabular}{lll}
\hline Contrast Balanced and Not Motivated to & $M$ & $S D$ \\
\hline Balanced and not motivated & -1.35 & 7.45 \\
Balanced and motivated & 1.93 & 6.88 \\
Focus and not motivated & 3.58 & 9.75 \\
Focus and motivated & 2.41 & 7.23 \\
\hline
\end{tabular}


Figure 8. Motivation by attention on integration confirmation bias.

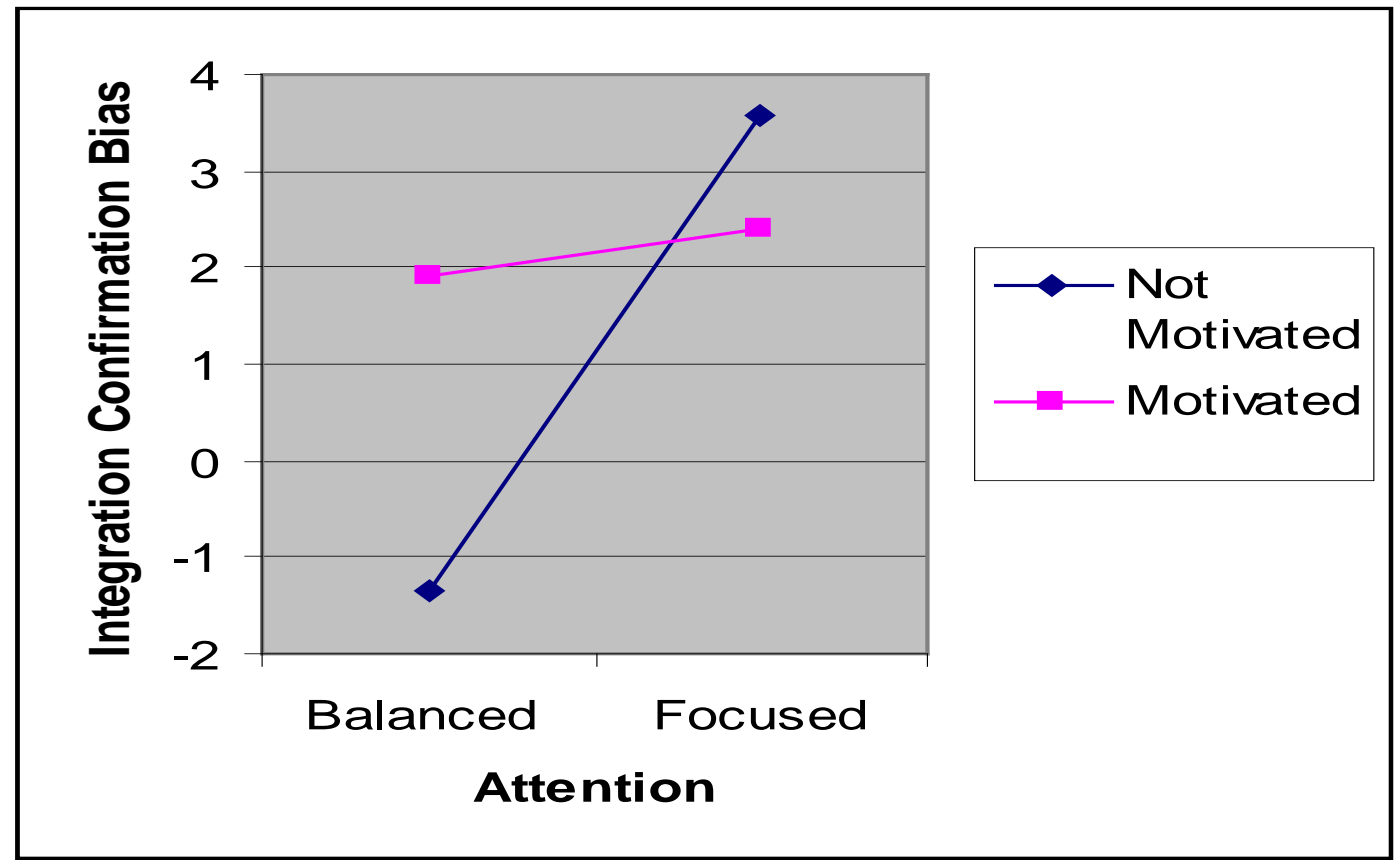

Additivity of phase biases. Exploratory Hypothesis \#12 was that participants confirmation bias $\left(\mathrm{DV}_{5}\right)$ would be less than their Sum-of-Phase-Biases $\left(\mathrm{DV}_{13}\right)$. Inconsistent with Hypothesis \#12, the paired samples t-test (pairing the confirmation bias of each participant with the sum of their phase biases) was not significant, $t(159)$ $=.028, p=.978$.

\section{Intuitive conditionals.}

Intuitive conditionals are the estimates participants have of the unchosen conditional probabilities. These hypotheses examine how participants estimate these values and if and how they use intuitive conditional probabilities in their posterior calculations. These analyses were limited to the 120 participants in the sequential conditions who provided every intuitive conditional. 
Results 170

Estimating the use of intuitive conditionals. Exploratory Hypothesis \#13a examined the probabilities used in calculating posteriors. This hypothesis was that Bayesian integration using the selected conditionals and intuitive conditionals would be better at predicting participants’ posterior probabilities than Bayesian integration using only the selected conditionals. The model using the selected conditionals and the intuitive conditionals that completed a diagnostic pair of the selected conditionals predicted the actual posteriors the best, $r(120)=.37, p<.001$. The model using all selected and intuitive conditionals predicted the actual posteriors second best, $r(120)=$ $.26, p=.005$. The model using only the selected pairs predicted the actual posteriors the worst, $r(120)=.19, p=.04$ (see Table 52).

To compare the accuracy of these three models in predicting participants’ posterior probabilities, the absolute value of the differences between the posteriors computed using each model and the participants' actual posteriors were calculated. These difference scores were compared using the Wilcoxan signed ranks test where the assumption of symmetry appeared to be satisfied, and the sign test where that assumption appeared to be violated. The model using only matching intuitive conditionals was better than the model using all intuitive conditionals (sign test), $Z(120)=4.69, p<.001$. Inconsistent with Hypothesis 13a, the model using only the real conditionals was significantly better than the model using the matching conditionals (signed ranks test), $Z(120)=2.36, p=.02$.

There is an apparent contradiction between these two sets of results, that use of intuitive conditionals and Bayesian Integration produces stronger correlations with, 


\section{Results 171}

and yet greater mean error from, the actual posterior. This contradiction can be resolved by examining the standard deviations of the models’ posteriors. Models using intuitive conditionals have more diagnostic pairs to integrate, and therefore produce greater variation from the midpoint. This can be seen in their higher standard deviations. In this study's situation, where most participants (77 out of these 120) provided a posterior between $40 \%$ and $60 \%$, a model that adjusts less will be more accurate in terms of difference between the model's posterior and the actual posterior. The model using all the intuitive conditionals and Bayesian integration had the strongest correlation, indicating that it predicts the correct order of the participants’ posteriors well. 
Results 172

Table 52

Comparison Accuracy of Models in Predicting Actual Posterior Estimates

\begin{tabular}{|c|c|c|c|c|c|}
\hline Intuitive & \multicolumn{2}{|c|}{ Model's Posteriors } & \multicolumn{2}{|c|}{ Absolute Value of the } & Model \\
\hline Probabilities Used & \multicolumn{2}{|c|}{ Correlation to } & \multicolumn{2}{|c|}{ Difference between the } & Posterior \\
\hline by Model & \multicolumn{2}{|c|}{ Actual Posteriors } & \multicolumn{2}{|c|}{ Model's Posterior and the } & \\
\hline (Integration & & & \multicolumn{2}{|c|}{ Actual Posterior } & \\
\hline \multicolumn{6}{|l|}{ Method) } \\
\hline & $r(120)$ & $P$ & $M$ & $S D$ & $S D$ \\
\hline All (Bayesian) & .26 & .005 & 22.44 & 20.12 & 29.81 \\
\hline Forming Matching & .37 & $<.001$ & 16.4 & 18.29 & 25.11 \\
\hline \multicolumn{6}{|l|}{ Pairs (Bayesian) } \\
\hline None (Bayesian) & .19 & .04 & 12.14 & 12.19 & 10.09 \\
\hline All (Regression) & .29 & .001 & 11.82 & 9.68 & 4.64 \\
\hline
\end{tabular}

Estimating the combining rule. Exploratory Hypothesis \#13b examined the combining rule used in calculating posteriors. In order to determine which integration strategy is a better overall predictor of actual posteriors, a regression using the average conditional (all intuitive and all selected) in each quadrant was used to predict the actual posterior. This regression equation was significant, $F(4,115)=2.68, p=.04$, and was used to estimate the posterior probabilities.

Exploratory Hypothesis \#13b was that the regression equation would predict participants' actual posterior probabilities better than Bayesian integration. Similar to 
Results 173

13a, the regression equation created posterior was compared to the Bayesian created posterior (both used all selected and intuitive conditionals). Consistent with hypothesis 13b the regression equation predicted the actual posteriors significantly better (sign test), $Z(120)=2.83, p=.005$, than did the model using Bayesian integration.

Interesting to note, that the posteriors made by regression did not correlate as strongly as to the actual posterior, $r(120)=.290, p=.001$, as did the model using Bayesian integration applied to the matched conditionals (see Table 46).

Unfortunately, a direct comparison between these two models is not possible due to their use of different numbers of conditionals.

Predicting the size of intuitive conditionals. Exploratory Hypothesis \#14 was that the size of intuitive conditionals will be larger for participants in the high conditionals conditions than the low conditionals conditions. Consistent with Exploratory Hypothesis \#14 participants in the high conditionals conditions ( $M=$ 49.68, $S D=14.34$ ) estimated higher intuitive conditionals than participants in the low conditionals condition $(M=26.73, S D=19.14), t(120)=7.51, p<.001, d=1.13$. This strongly indicates that participants use the size of actual conditionals in estimating intuitive conditionals. 
Surprise hypotheses. These hypotheses examined the idea that surprise leads to deeper and less biased thinking. This was a critical component of the assumption that the combination of low conditional (the surprise) combined with sequential feedback (an opportunity to change search strategies) would result in lower bias.

Exploratory Hypothesis \#15. Exploratory Hypothesis \#15 was that participants who were in the focused and/or motivated conditions and reported being surprised would select less information conditioned on Shell Island than those not surprised. Inconsistent with Exploratory Hypothesis \#15 the $t$-test was not significant, $t(118)=$ $.40, p=.69, d=.08$ (see Table 53).

Exploratory Hypothesis \#16. Exploratory Hypothesis \#16 was that participants who were in the focused and/or motivated conditions and reported being surprised would select less information hinted to favor Shell Island than those not surprised. Inconsistent with Exploratory Hypothesis \#16 the $t$-test was not significant, $t(118)=$ $.35, p=.73, d=.06$ (see Table 53). 
Results 175

Table 53

Descriptive Statistics for Surprised and Not-Surprised Participants in Motivated and / or Focused Conditions

\begin{tabular}{lcccc}
\hline Surprised (n) & \multicolumn{2}{c}{ Hypothesis Focus Bias } & \multicolumn{2}{c}{ Predictor Selection Bias } \\
& M & SD & M & SD \\
\hline Surprised (47) & 2.49 & .80 & 2.30 & .78 \\
Not Surprised (73) & 2.42 & .90 & 2.25 & .80 \\
\hline
\end{tabular}

The failure of these surprise hypotheses to predict search biases, demonstrates why the low conditionals condition failed to "cure" biases. The low conditionals were supposed to reduce bias by surprising participants, but surprise did not reduce bias. 
Discussion 176

\section{Discussion}

\section{Primary Goals}

The primary goals of this study were to address four problems in the literature: the imprecise and multiple definitions of terms relating to confirmation bias, the lack of any quantification of the confirmatory effect against a normative interval scale outcome, the absence of studies that examine phase interactions, and the absence of research on predictor selection bias (outside dissonance theory research).

In reviewing the literature, a series of definitions was proposed to increase the precision of the terms used in this area, in order to achieve the first goal. This study has taken the first small step towards its second goal, measuring the size of confirmation bias with reference to a normative interval scale outcome. This goal was hindered by the way in which the study was designed, in which efforts to establish controls rendered the manipulations less effective. (See below Limitations of internal validity from manipulation problems). Nevertheless, if the proposed terms are accepted by the research community and research follows this example of using a normative interval scale outcome, then results will, in the future be more comparable across studies. This in turn should increase communication among researchers working across the seven related paradigms. Additionally, achievement of the first two goals in combination would assist in determining the relative importance of the various forms of confirmation bias, which, in turn, would inform the prescriptive research as to which biases are the most important to be on guard for. 
This study has also taken the first steps towards its third goal, examining phase interactions by studying both search and integration phases in the same experiment. First, this study directly tested the common unstated assumption in the literature that the phases are either completely independent or completely dependent, and therefore can be examined separately. The strong correlation $(r=.81)$ between the use of focal search and focal integration strategies is powerful evidence that these two processes are interrelated and eliminates the possibility of complete phase independence. Despite this extremely high correlation, the phases are not completely dependent, either, for five reasons. First, because this correlation was between categories of strategies (focal, unbiased, and anti-focal), there was a lot of variation within these categories. For example, there were three different search strategies that were classified as focal and six different integration strategies that were classified as focal. The 18 combinations of these strategies produce vastly different posteriors. Second, the correlation between focal search and focal integration approaches randomness $(r=.02)$ when participants who selected probabilities conditioned exclusively on one island (and therefore cannot be focal in integration on the other island) were excluded. Third, the change in the attention-by-motivation interaction between phases (see over-biasing effect below) indicates a reduction in participants' focus in the integration phase. Fourth, the finding of hypothesis-focus bias in the search phase but not finding the parallel hypothesisfocus integration bias in the integration phase again indicates a reduction in participants' focus in the integration phase. Fifth, many of the participants searched optimally but used the Biased Bayesian integration strategy. Since the phases are not 
completely independent or dependent, then prior research examining only one of the two phases while making claims about confirmation bias results needs to be critically re-examined.

The fourth goal, that of examining predictor selection bias, was both less ambitious than the others and yet turned out to be more difficult. Although dissonance theory research has extensively examined predictor selection bias, conditional selection paradigm research has never attempted to examine it in the integration phase. This oversight may be due to lack of interest, or due to the difficulty in establishing hints as to the direction of the diagnosticity of the dimensions without making these hints normatively relevant in the integration phase.

This study attempted to overcome this difficulty by creating a situation where the hints were clear as to their direction but were not a normative factor in integration. There are three reasons why they are not normative factors. First, the hints were balanced (four favoring each of the two hypotheses) and therefore, as a set, nondiagnostic. Second, anyone following a normative search strategy would select conditionals evenly from those hinted to favor each hypothesis thus preserving this balance. Third the hints were deliberatively vague as to whether they were diagnostic or pseudodiagnostic (“...characterizes the current clay pits of...”).

No results met the .05 level on predictor selection bias or related biases. However, since previously found biases were not replicated, it is concluded that this was due to a weak manipulation, we therefore, cannot rule predictor selection bias out. There was one new finding in this area of predictor preference: the use of the Biased 
Bayesian integration strategy (the use of only the pair favoring the focal island in integration by participants who had searched optimally). Both attention and motivation had nearly significant effects on the use of this Biased Bayesian strategy. This form of predictor-focus integration bias has never been considered before, and if established in subsequent research, would show a new way that participants who are unbiased in one phase (search) can be powerfully biased in another (integration).

\section{Results Summary}

The results indicate that the attention manipulation had both focal and motivational effects in both the search and integration processes, but these did not produce an effect on the resulting posterior probability. In contrast, the motivation manipulation had only focal search effects. Additionally attention and motivation manipulations appear to interact, but only in the integration phase.

This study's unique design of providing hints as to the direction of diagnosticity of a dimension and of examining both search and integration phases provided the ability to examine more combinations of search and integration strategies. This study surprisingly found that participants were rarely using singlequadrant search strategies (33) and were frequently using information about both islands in integration (127). Additionally, there was some evidence for the use of the Biased Bayesian integration strategy.

Finally, this study is the first to examined participants' intuitive conditionals. Strong evidence was found that participants estimate of the size of the unchosen conditional probabilities was affected by the size of the chosen conditionals (positive 
Discussion 180

relationship). Additionally, it appears that participants’ intuitive conditionals affect their estimate of the posterior probability.

\section{Results Categorized by Independent Variable}

The following six sections discuss the results by focusing in turn on each of the four independent variables, the interaction between attention and motivation, and a comparison of the size of the effects of the attention and motivation manipulations. For ease of understanding main effects will be presented before interaction effects.

Attention main effects. The attention manipulation was found to increase significantly the use of focal search strategies ( $p=.04$; exploratory hypothesis \#3) and integration confirmation bias ( $p=.03$; exploratory hypothesis \#11). Attention was found to nearly significantly increase hypothesis focus bias $(p=.07$; exploratory hypothesis \#1), focal integration strategies ( $p=.08$; exploratory hypothesis \#9), and the use of the Bias Bayesian integration strategy ( $p=.09$; research hypothesis \#2).

However, attention was found to be not significant in affecting predictor selection bias (research hypothesis \#1) or its integration phase equivalent, predictorfocus integration bias (exploratory hypothesis \#7). These findings were smaller in size and narrower in scope than expected (see Interval Validity Section for a discussion).

Additionally, this study failed to replicate prior findings of pseudodiagnosticity (exploratory hypothesis \#2), as well corresponding integration phase biases of Hypothesis-Focus Integration Bias and pseudodiagnostic integration (exploratory hypotheses \#6 and 8) (Doherty, et al., 1979; Doherty, et al., 1981; Beyth-Marom \& Fischhoff, 1983; Fischhoff \& Bar-Hillel, 1984; Ofir, 1988; Mynatt, et al., 1993). 
Motivation main effects. The motivated manipulation was found to increase significantly the use of focal search strategies ( $p=.04$; exploratory hypothesis \#3) and nearly significantly increase hypothesis focus bias ( $p=.07$; exploratory hypothesis \#1) and the use of the Bias Bayesian integration strategy ( $p=.07$; research hypothesis \#2). Motivation was found to be non-significant in affecting most search phase variables, including: predictor selection bias (research hypothesis \#1), pseudodiagnosticity (exploratory hypothesis \#2), and search confirmation bias (exploratory hypothesis \#10). Similarly, motivation was found to be non-significant in affecting most integration phase variables, including hypothesis-focus integration bias (exploratory hypothesis \#6), predictor-focus integration bias (exploratory hypothesis \#7), and pairs used in integration (exploratory hypothesis \#8).

These findings were smaller in size and narrower in scope than expected (see Limitations of internal validity from manipulation problems for a discussion) as the consistent effects found in the dissonance theory paradigm (Frey, 1981, 1986; Ditto \& Lopez, 1992; Pinkley, et al., 1995; Frey, et al., 1996; Johnston, 1996; Russo, et al., 1996; Luce, et al., 1997; Lundgren \& Prislin, 1998; Schulz-Hardt, et al., 2000; Simon, et al., 2001), despite the parallel situations. This failed to extend dissonance theory paradigm to the Bayesian conditionals selection paradigm.

Motivation and attention interaction. The attention and motivation manipulations were expected to be sub-additive by producing similar levels of bias regardless of whether a participant was assigned a focal condition, a motivated condition, or both (see Expected effects of independent variables). However, this sub- 
additive interaction was not consistently found in this study. Interestingly, a pattern was found for this interaction. In the two search phase dependent variables where significant or nearly significant results were obtained (hypothesis focus bias and search strategy), these independent variables appeared to have additive effects. In contrast, in the two integration phase dependent variables where significant or nearly significant results were obtained (integration confirmation bias and integration strategy), these independent variables appeared to have sub-additive or even overbiasing effects. Participants appear to grow more cautious or less biased (perhaps because they become aware of the manipulations) as they reached the integration phase of this study.

Motivation and attention comparison. The study compared the sizes of the effects of these two sources of bias (attention focus and motivation bias). Despite the weak manipulations (and the inherent problems in comparing the strength of manipulations of different variables), this study found the effect of attention to be greater than the effect of motivation on hypothesis focus bias $(p=.09$; exploratory hypothesis \#4). In contrast, motivation was no found to be significantly greater in effect on predictor selection bias than attention (exploratory hypothesis \#5).

Timing of feedback. Due to the failure of random assignment, all sequential participants were dropped from analyses, and therefore it was not possible to examine the effects of this variable (see the Interval Validity Section for a discussion).

Size-of-conditionals. This independent variable was included to examine two possibilities. The first possibility was that a combination of low size-of-conditionals 
and sequential search would be unbiasing. Because of the selection differences in the timing of feedback variable this possibility could not be examined. The second possibility was that size-of-conditionals affected participants' intuitive conditionals. This relationship was highly significant $(p<.001$; exploratory hypothesis \#14) and in the expected positive direction.

\section{Results Categorized By Dependent Variable}

The following five sections discuss the results, each focusing on one of five categories of dependent variables in turn: search phase, integration phase, surprise, intuitive conditionals, and search and integration strategies.

Search phase. Focal attention and the motivated conditioned produced their expected increase in hypothesis focus bias and the use of focal search strategies (as discussed under main effects). Additionally, the small conditionals condition surprisingly increased the biasing effect of focal attention. Unfortunately, the study failed to replicate many prior findings of the effect of attention on pseudodiagnosticity (Doherty, et al., 1979; Doherty, et al., 1981; Beyth-Marom \& Fischhoff, 1983;

Fischhoff \& Bar-Hillel, 1984; Ofir, 1988; Mynatt, et al., 1993).

One notable finding was that a surprisingly large proportion of the participants searched for at least one diagnostic pair (133 out of 324) and approximated the use of Bayes' theorem in integration (88 of 324). These participants selected significantly more pairs than chance, while in contrast, Doherty, et al. found participants selecting significantly fewer pairs than chance. It is impossible to know whether this is a cohort effect, a sampling effect, or an effect of the difference in the scripts. 
Integration phase. Fewer significant results were found in the integration phase than the search phase. Attention was found to increase significantly integration phase confirmation bias ( $\mathrm{p}=.03)$. Additionally, attention and motivation had a nearly significant overbiasing interaction on the use of the Biased Bayesian integration strategy. (The overbiasing interaction was participants both focal attention and motivated conditions demonstrating less bias to those participants in either one biasing condition alone). In contrast attention and motivation had a sub-additive affect on integration confirmation bias. (The sub-additive interaction was participants both focal attention and motivated conditions demonstrating equal bias to those participants in either one biasing condition alone).

Surprise. Similarly, two critical assumptions of this study were that the low conditionals would prove surprising, and that surprised participants would be "cured" of the biasing effects of the focal and motivated conditions. Unfortunately, both of these assumptions were not supported in this study. First, low conditionals did not prove to be very surprising. Only 39\% of participants in the low conditional probabilities condition reported being surprised by the size of the conditional probabilities (in contrast to $33 \%$ of the high conditionals participants). Second, those who reported being surprised were more, rather than less, biased (although not significantly), in terms of both hypothesis focus bias and predictor selection bias (exploratory hypotheses \#15 and16). These two unexpected findings regarding surprise combined to virtually eliminate any significant finding regarding size-ofconditionals. This failed to extend Doherty’s (Doherty, et al., 1979) and Ofir's (1988) 
findings that providing disconfirming diagnostic feedback helped participants search diagnostically. This goal was not achieved since there was only one significant interaction between the size-of-conditionals and attention or motivation.

Intuitive conditionals. One of this study's strongest findings was that the size of the selected conditional probabilities had a positive relationship with the size of the participants' intuitive conditionals. This demonstrates that participants are estimating these values and are influenced by what they do know about the situation.

Another interesting finding was that the model using the actual conditionals plus their complementary paired intuitive conditionals (given Bayesian integration) provided the strongest correlation to the actual posteriors ( $r=.37)$. In contrast, the nonparametric tests indicated the opposite, that regression was better than Bayes’ Theorem. These conflicting results between the correlation method (which indicated that the intuitive conditionals combined with Bayes' theorem was the best) and the non-parametric methods (which indicated that regression was better than Bayes' theorem and that using no intuitive conditional probabilities was better than using any) may be explained by the difference in the criteria. The regression model had lower variation (than the Bayesian models) and therefore resulted in more posteriors close to $50 \%$. In this situation, anything close to 50\% predicted well, since most participants stayed near 50\%. However, the models using Bayesian integration and including some or all intuitive conditionals had higher correlation coefficients to the actual posteriors, indicating that they better captured the variation of participants' posteriors. The regression model may reflect participants using the anchoring and adjusting heuristic 
proposed by Tversky and Kahneman (1974) (anchoring on the 50\% base rate and adjusting from there) and the small size of the adjustment. The Bayesian model (Bayesian integration applied to all the intuitive conditionals) may better reflect how people adjust from the anchor, but may overstate the size of the adjustment. In situations where the anchor is not so fixed (due to non-50\% base rate and consistent diagnosticity of the conditional probabilities) the intuitive conditionals model with Bayesian integration might predict even better.

This provides additional evidence for participants using Bayesian or QuasiBayesian thinking. This support is limited due to the contrasting findings (as to whether regression or Bayes predicted posteriors better), and the fact that these are between-subjects group-level findings, and therefore it is hard to draw individual-level conclusions from them.

Additionally, these results provide mixed evidence that the intuitive conditionals were somehow involved in the integration process and that people are semi-Bayesian in their integration. Unfortunately, these intuitive conditional results need to be taken cautiously, since the data were collected after the posterior judgments had been made and may reflect some hindsight bias.

Participants' use of intuitive conditionals could provide an explanation for participants' poor performance in many decision making tasks. When making real decisions, people often are familiar with the situation. This familiarity could help them in at least three ways. First, familiarity would provide a hint to the direction of the diagnostic effect of each datum (i.e. the hints in this study that this mineral would 
favor Shell or Coral). Second, familiarity would help people to estimate the size of conditional probabilities that they do not have exact figures for (i.e. the diagnostic ratio of unchosen conditionals in this study). Third, familiarity would guide them to the importance and diagnostic direction of the value of each conditional probability (i.e. the diagnostic impact of a high or low conditional probability would have on their posterior probability). The artificial and abstract tasks of most laboratory studies in this field may cause some of the participants' poor performance, by denying them their usual familiarity with the task and situation.

Search and integration strategies. This study created a taxonomy of all possible search strategies and measured their relative frequency of use. Of interest is the rarity (only 33 participants) of participants who choose information all from the same quadrant (all probabilities conditioned on a single island and hinted to favor a single island) indicating that for the vast majority of participants information from more than one quadrant was relevant. Similarly, this study created a list of integration strategies and a classification system that proved acceptably reliable. Of great interest among the integration strategies was the large number of participants who used the Biased Bayesian (32), Bayesian (56), and ratio of conditionals strategies (35). These three strategies all use information from both islands in ways that are at least close to Bayes' Theorem. This study found a larger proportion of Bayesian participants than did Doherty, et al. (1979) although direct comparisons are difficult due to differences in the task and script. 


\section{Limitations}

This study suffers from several limitations that limit the scope and both the internal and external validity of the study.

Limitations from the scope of the study. Several potential research questions were left outside the scope of this study. Most importantly, despite being the first study to examine the interactions between the search and integration phases, this study avoided examining interactions between this and other parts of the decision making process. Hall (1989) outlined eight steps in the systems methodology for decision making (problem definition, value system design, systems synthesis, systems analysis, optimization, decision making, planning for action, and building and maintenance functions). In the decision making literature these same steps are commonly referred to as problem definition, identification of values, identification of alternatives, evaluation of impacts, maximizing individual alternatives, choice among alternatives, and implementation (merging the last two systems methodology steps).

Of these, only two were involved in this study, systems analysis (the search and integration of information) and to a lesser extent decision making (the final decision). The first four of Hall's steps were not examined, because participants were simply provided at the outset what would have been the results of Hall's first four steps (problem definition, value system design, systems synthesis, and optimization). Similarly, Hall's last two steps (planning for action and maintenance functions) were ignored completely. An obvious limitation of this research (and an area for future 
research) was any interaction between the excluded six phases of Hall’s decision making process and the two phases studied.

Limitations of internal validity from manipulation problems. The failure of the attention and motivation manipulations to produce their expected effects interfered with several of this study's goals (with the notable exceptions of significant results on search strategies and integration confirmation bias). There are eleven possible explanations to this failure to replicate both the pilot study and many prior research findings.

First, the length and complexity of the script and the number of process questions could have deceased participants' interest, motivation, and effort, leading to random responses and/or 50\% posterior estimates. (93 participants gave exactly 50\% as their posterior and 15 more were within 1\%).

Second, two features of this study encouraged balanced attention and may have undermined the attention manipulation (the pilot study and most other studies used non-equal base rates). The even base rate (required for the unique optimal search strategy) encourages participants to treat both islands equally. Similarly, use of the table format when making search selections, again, treats both islands equally and has been found to promote balanced thinking (Ward \& Jenkins, 1965).

Third, the balanced conditionals (again required for the unique optimal search strategy) could have led many non-Bayesian integration strategies to result close to $50 \%$. In fact, in the situation presented in this study both the participants who use the normative search and integration strategies (balanced Bayesian search and Bayesian 
Discussion 190

integration) and the most unmotivated (“I don’t know”, therefore 50\%) might be expected to produce the same posterior. The balanced conditionals themselves may cause an expectancy effect, where participants realize that the experimenter is looking for bias and that $50 \%$ is the fair or even or correct answer.

Fourth, the lack of conflict among the base rate, the conditional probabilities, and the hints may encourage less deep thinking and a 50\% posterior (which is the exact posterior chosen by 93 of the 324 of the participants). Ofir's (1988) found that conflicting information encouraged the use of false alarm rate information (deeper thinking).

Fifth, the existence of an over-biasing effect would reduce the effect of the attention and motivation manipulations by eliminating the biasing effects of these manipulations for the $25 \%$ of participants who receive the combined conditions of focal attention and motivated conditions. This lowered the power of all analyses involving these two manipulations using the original testing design (combining the IVs into one dichotomous variable, biasing and non-biasing conditions).

Sixth, some dependent measures such as hypothesis focus bias and predictor selection bias were not very sensitive (since there were only five possibilities of numbers of conditionals selected in a category 0-4) and with most participants selecting two probabilities conditioned on each island (71.6\%) and most participants selecting two probabilities hinted to favor each island (69.4\%). In contrast Doherty in his original study (Doherty, et al. 1979) used a situation that produced a 0-6 scale. 
Discussion 191

Seventh, the power of the study was reduced by the pre-manipulation selection differences that caused the eight simultaneous conditions (and half the participants) to be dropped. These subjects demonstrated differences in their search phase behavior. These differences appear to be caused by the size of the conditional variable, which the participants should not have yet seen. This indicates that many of these subjects might have misunderstood the instructions and chosen their conditional probabilities sequentially or cheated and changed their selections after the fact. This resulted in half the participants being dropped.

Eighth, the increase in the proportion of Bayesian participants may make research in this area less statistically sensitive. It appears to be a safe assumption that Bayesian participants are more aware of the need to examine probabilities conditioned on both hypotheses, and by extensions that they would be more aware of the need to be fair in selection information hinted to favor both hypotheses. Therefore, they would be less vulnerable to manipulations of their attention and motivation.

Ninth, the minimal effect of the low conditionals to generate surprise, and the minimal effect of surprise on participants' actions appears to have interfered with the original testing design (combining the IVs into one dichotomous variable, biasing and non-biasing conditions). Additionally, the low conditionals may have generated apathy, anger or confusion rather than the intended surprise in participants when they did not receive the feedback they were expecting. For example 12 low conditionals participants failed to answer the surprise question while only 7 high conditionals participants failed to answer the surprise question $(p=.13$, $\mathrm{df}=322)$. 
Tenth, the script for the pilot study (Borthwick \& Anderson, 2001) was in a setting that participants would be familiar with (hiring a new employee) as opposed to the current script that focused on a more abstract problem (but is more similar to the one used by Doherty, et al., 1979). The intuitive conditional results support the idea that familiarity with the context could affect performance.

Eleventh, the pilot study (Borthwick \& Anderson, 2001) was conducted over the summer, whereas the current study was conducted during the academic school year. Summer students may be more motivated participants and / or more vulnerable to biases (they chose diagnostic pairs at a rate less than chance in contrast to these participants who chose diagnostic pairs at a rate more than chance).

Technical limitations. This study additionally has three technical limitations. First, the hints were all accurate and all of the hints, the probabilities, and the base rate were perfectly balanced between alternatives (as was required for creating a single normatively correct answer). If participants identify any of these characteristics, their identification may reinforce their tendency to respond with a 50\%-50\% final estimate (which in this case happens to be correct). This problem would work against rejection of the null hypotheses.

Second, the definition of predictor selection integration bias, while not a limitation in this study because the hints were all accurate, could be a limitation in the general case when hints might not be accurate. If the hints were not accurate then the hints and the data would disagree as to which predictors would support the focal 
Discussion 193

hypothesis. Then it would no longer be clear which dimensions should be considered indicative of predictor selection integration bias.

Third, several dependent variables were not continuous and had as few as five values. This restriction of range would work against rejection of the null hypotheses.

Limitations of external validity due to the laboratory setting. Finally, there were at least five possible limitations of this study that stem from its laboratory experimental design.

First, the artificial situation may have made participants less biased by making them more cautious in order to avoid looking "bad". This caution may have led them to minimize the distance by which they would revise their probability estimates, resulting in more posterior probabilities around 50\%. This problem would generally bias this research against rejecting the null hypotheses. This effect is unlikely or small because prior published lab studies commonly achieved significant results.

Second, the fact that participants were isolated from other people may have distorted the results, although research on group decision making has demonstrated some of the same biases as individual decision making (Schulz-Hardt, Frey, Luthgens, \& Moscovici, 2000). Consulting others who may have a different attention focus and different motivations could reduce these biases. This problem would generally bias this research towards rejecting the null hypotheses, and might also create an external validity problem. Again, this effect is unlikely or small because prior published lab studies did commonly achieve significant results. 
Third, other reactivity effects were possible since participants knew their decision making process was being studied. One possible effect of reactivity would be to encourage participants to use in integration all the information they selected in the search phase. Because if participants did not use all their selected information, it would be admitting that they made a mistake in their search selections and had chosen useless information. A second example would be participants changing their nonnumeric strategies to a numeric strategy in an attempt to use the "correct" integration method.

Fourth, participants may have responded randomly due to low motivation and a desire to avoid cognitive effort on this difficult task (the 97 participants who chose exact $50 \%$ may be an indication of this).

Fifth, any results are limited to the research paradigm employed (Bayesian conditionals selection paradigm), the situation, and the parameters employed. As just one example, this study asked participants to choose 4 of 16 conditional probabilities, the fact that participants then had 4 conditional probabilities in addition to the base rate, made it likely that participants who would have used an additive strategy with fewer numbers to work with, would switch to another strategy when they realize that adding four or five probabilities together would result in a probability above $100 \%$ and therefore cannot be the correct integration strategy. No other researcher has tried to categorize individual participant’s integration strategies. 
Discussion 195

\section{Implications}

Multi-phase research. Eventually, future research could address some of these limitations, most notably by including all eight of Hall’s (1989) system methodology steps. This research would require a less structured problem, which would entail a more difficult statistical analysis. Such research would probably focus on defining and/or classifying patterns or styles of decision making in individuals rather than averaging across individuals. Similarly, moving the study out of a laboratory environment would resolve the five potential limitations listed above for this study due to its laboratory setting. However, the use of field studies is largely prevented by the logistical difficulties of observing decision making in a field study. Some of the limitations of a laboratory study can be addressed while still in the laboratory environment, such as having married couples perform both joint and individual decision making.

Once studies are expanded to all eight of Hall’s (1989) system methodology steps, other biases that have sometimes been labeled confirmation bias (and were excluded from this study) could be included. These include biases in the interpretation of (Kelley, 1950) and memory for information (Perkins, et al., 1991). These two biases could interact with the biases explored in this study. For example, when memory is involved, participants may use information from dimensions that are normatively anticonfirmatory (e.g. predictor-focus integration anti-bias) but they misremember the information to be confirmatory. 
Technical improvements for future laboratory research. There are at least five ways to make technical improvements to laboratory research in this area.

The first way would be to strengthen the manipulations. One critical failing of this study was the weakness of the manipulations. In order to overcome this problem several options are available. For the attention manipulation, the use of an uneven base rate and/or the presentation of the search choices in a form other than a table would strengthen the attention manipulation. For the motivation manipulation, more real world motivations could be stronger. These could include a promise of a real life reward for the desired outcome (e.g. getting out of the study earlier, small cash reward, more extra credit points) or the use of a decision involving a topic participants have an existing strong opinion on (e.g. Iraq War or Abortion). Another technique to maximize the size of the bias would be to ask participants to deliberately be biased. This technique has been used extensively in personality testing for employment purposes (see Viswesvaran \& Ones, 1999 for a meta-analysis and review).

The second way to improve the study would be to automate the study through computer programming. The use of an automated script could prevent some of the more common participant mistakes, such as selecting more than 4 conditional probabilities and providing less than all 12 of the intuitive conditional probabilities.

The third way to improve the study would be change it to a within-subjects design. The most interesting participants for this topic are the least numerically oriented. Unfortunately, these participants’ integration strategies proved very difficult to classify without any mathematical notations to guide our understanding of their 
thinking. In a within-subjects design, individual integration strategies can be inferred more accurately from their repeated judgments.

The fourth way to improve the design would be to cut up the study into a series of smaller experiments. While we need to understand the whole system, the length and complexity of this study may have contributed to some of its failing and therefore, simplifying the study may improve the quality of the resulting data. This could be done by running an initial experiment limited to the search phase with just the attention manipulation. Each succeeding experiment could build on the prior study by adding or changing one feature (i.e. changing how attention is manipulated or by adding a motivation manipulation). This research plan would have avoided some of the problems experienced by this study (e.g. failure of attention and motivation to consistently cause biases, and failure of surprise to reduce biases).

The fifth way to improve the study would be to improve the hints. The hints in this study may have been both artificial and weak. The pilot study used an employee selection cover story, in which the hints were whether or not the job applicant passed each test. This form of a diagnostic hint is simultaneously understandable (pass is good, failed is bad), familiar, and subtle (no need for a long explanation as to what passed means). Additionally, these hints could easily be modified to be strong or weak in their diagnosticity.

Future research topics. The results of this study suggest at least seven implications for future laboratory research in this area. 
Phase interactions. The most important implication of this study is that future research must be careful about making conclusions about effects in phases that are not examined. The search and integration phases were shown to be both partly-dependent on each other, and partly-independent (see Primary Goals for a discussion of the evidence of partial dependence). Thus, cross-phase generalizations need to be made cautiously, if at all.

Intuitive conditionals. The finding that intuitive conditionals are used in Bayesian integration has three important research implications. First, research should not be conducted in this area without gathering intuitive conditionals. Second support is provided for Gigerenzer's arguments that the degree to which cognitive errors found in laboratory experiments can be a product of the artificial environment. If participants use intuitive controls then it is not automatically a cognitive error for people to search pseudodiagnostically. Third, this raises questions regarding other findings of cognitive errors from laboratory experiments. Future research could further investigate this phenomenon, and the related issue of the participants' familiarity with the context.

Studies could ask participants about intuitive controls after actual conditional probabilities are provided but before their posterior probability is collected. Similarly, intuitive conditionals could be gathered before the search phase selections are made or after the selection are made but before feedback is provided. Another way to gauge their impact would be to vary the number of dimensions while keeping the number of selections constant. This would determine whether the number of unexamined dimensions of intuitive conditionals (diagnostic pairs of intuitive conditionals) affects 
the decision process. If participants can be accurately classified according to their integration strategies, then their use of intuitive conditional probabilities could be more effectively examined.

Relative size of biases from attention and motivation. This study found weak support that attentional biases are greater than motivational ones. (Exploratory Hypotheses 4 and 5 found that for both attention and motivation the effects were greater on focal search bias than predictor selection bias. Additionally, attention produced more and stronger effects than did motivation.) Therefore, research should focus on attentional processes more than on motivational ones.

Surprise. This study’s failure to replicate prior findings regarding surprise indicates that surprise may not be inherently debiasing, or that what other researchers labeled as surprise was something else. Future research employing surprise as a variable should use a manipulation check to ensure that they are actually surprising the participants.

Overbiasing. This study's finding of an overbiasing effect between attention and motivation could either indicate a demand characteristic or some other mechanism. One possibility is that what was here found and labeled as overbiasing, may in fact be related to conservatism, which is the tendency to under-adjust for new information. The participants' initial positions are assumed to be the base rate. Therefore any adjustment from it, whether caused by attentional or motivational processes, would have to work against an inherent resistance to change. Future 
Discussion 200

research should determine the extent of overbiasing, and the degree it is related to broader decision making concepts.

Predictor selection bias and the Biased Bayesian Integration Strategy. The findings regarding predictor selection bias and the biased Bayesian integration strategy call for a re-examination and interpretation of prior findings. Even in situations where the research did not intentionally include hints as to the diagnostic direction of information, participants may have their own ideas / prejudices. The use of cover stories with familiar situations (e.g. the color of a cab) in prior studies may have activated participants' biases as to the diagnosticity of a dimension. This possibility should be investigated.

Increase in frequency of Bayesian participants. The finding of a greater number of Bayesian participants raises a caution for future research. Since Bayesian participants (133 participants selected at least 1 diagnostic pair) are presumably more resistant to biases, an increase in the proportion of Bayesian participants will lower the effect size of any biasing manipulation in a between-subjects design. Research may have to compensate through increasing the strength of their manipulations, or running more participants.

Specific future studies. Three specific studies are suggested by this research. A first study could systematically manipulate the base rate in order to examine the base rate's attentional impact on predictor selection bias, hypothesis focus bias and pseudodiagnosticity. This would help determine why the results of this study differ from its pilot study. 
Discussion 201

A second study could examine the interaction between attention and motivation. This study found evidence for additive, sub-additive (when the effect of two variables together is less than the combination of the two effects separately) and over-biasing (when the effects of two variables together is less than either of the effects separately) effects of attention and motivation. The interaction appears subadditive or over-biasing in the integration phase while additive in the search phase. Determining why this interaction effect changes would be useful.

A third study could examine the possibility of mediator relationships among the variables. The current study's definition of confirmation bias (as a confirmatory effect on the posterior) implies that the effects of the independent variables on confirmation bias are mediated through the three search phase biases: pseudodiagnosticity, hypothesis focus bias and predictor selection bias. Similarly, these three search phase biases may (also) mediate the relationship between the independent variables and their corresponding integration phase biases (pseudodiagnostic integration, Hypothesis-Focus Integration Bias, and predictor selection integration bias). The current study did not find consistent patterns of search phase biases causing parallel integration phase biases.

Researchers have always assumed that search phase biases produced a confirmatory effect, but they now need to not only demonstrate the confirming effect, but also the process of how a search phase bias becomes transmitted across phases.

Implications for practice. Implications for practice can be divided into those for decision analysts and those for untrained decision makers. Decision analysts’ use 
Discussion 202

of software for computational purposes ensures that appropriate probabilities will be obtained and that they will be integrated optimally. However, decision analysts and their software are still vulnerable to errors in identifying diagnostic information and in judging probabilities. This study has two findings that can impact these areas of vulnerability. First, this study showed that the judgment of a conditional probability can be affected by the size of other conditional probabilities. Second, this study also highlighted the possibility that the diagnostic information considered might be vulnerable to predictor selection bias.

For decision makers unaided by decision analysts and decision analysis software, this study provides three issues to be aware of in addition to those for decision analysts. First, attention may be a more important bias to be aware of than motivation, so tables or trees should be used to equalize attention. Second, they should be aware that they need to find and use diagnostic pairs of conditional probabilities. Third, despite the use of an optimal search strategy and integration with Bayes’ Theorem, bias is still possible (the Biased Bayesian integration strategy). Therefore, decision makers should ensure that all diagnostic pairs are integrated. Fourth, they should be aware that the size of intuitive conditionals may affect their judgment of the posterior probability, and therefore should be careful about any intuitive conditional probabilities excluded from the analysis. 
References 203

\section{References}

Allan, L. G. (1993). Human contingency judgments: Rule based or associative? Psychological Bulletin, 114, 435-448.

Alloy, L. B., \& Tabachnik, N. (1984). Assessment of covariation by humans and animals: The joint influence of prior expectations and current situational information. Psychological Review, 91, 112-149.

Anderson, B. F., Deane, Donald H., Hammond, K. R., McClelland, G. H., \& Shanteau, J. C. (1981). Concepts in judgement and decision research. New York: Praeger Publishers.

Anderson, B. F., Gaffuri, A. \& Mooris, R. K. Treatment-condition bias in the judgment of covariation. Unpublished Manuscript.

Arkes H. R., \& Harkness A. R. (1983). Establishing contingency between two dichotomous variables. Journal of Experimental Psychology: General 112, 117-135.

Bar-Hillel, M., \& Fischhoff, B. (1981). When do base rates affect predictions? Journal of Personality and Social Psychology, 41, 671-680.

Baron, J., \& Ritov, I. (2004). Omission bias, individual differences, and normality. Organizational Behavior and Human Decision Processes, 94, 74-85.

Baron, J., Beattie, J., Hershey, J. C. (1988). Heuristics and biases in diagnostic reasoning: II. Congruence, information, and certainty. Organizational Behavior and Human Decision Processes, 42, 88-110. 
Baron, J., \& Hershey, J. C. (1988). Heuristics and biases in diagnostic reasoning: I. Priors, error costs, and test accuracy. Organizational Behavior and Human Decision Processes, 41, 259-279.

Baron, J., \& Hershey, J. C. (1988). Outcome bias in decision evaluation. Journal of Personality and Social Psychology, 54, 569-579.

Bassok, M., Trope, Y. (1983). People's strategies for testing hypotheses about another's personality: Confirmatory or diagnostic? Social Cognition, 2, 199-216.

Beattie, J., \& Baron, J. (1988). Confirmation and matching biases in hypothesis testing, The Quarterly Journal of Experimental Psychology, 40, 269-297.

Beyth-Marom, R., \& Fischhoff, B. (1983). Diagnosticity and pseudodianosticity, Journal of Personality and Social Psychology, 45, 1185-1195.

Borthwick, G. L., \& Anderson, B. F. (2001, August). Pseudodiagnosticity and confirmation bias. Poster session presented at the $109^{\text {th }}$ American Psychological Association Conference, San Francisco, CA.

Broadbent, D. E. (1975). The magic number seven after fifteen years. In A. Kennedy \& A. Wilkes (Eds.), Studies in long term memory, (pp. 3-18), NY: John Wiley.

Brownstein, A. L. (2003). Biased predecision processing. Psychological Bulletin, 129, 545-568.

Bruner, J. S., Goodnow, J. J., \& Austin, G. A. (1956). A study in thinking. New York: Wiley. 
References 205

Dawson, E., Gilovich, T., \& Regan, D. T. (2002). Motivated reasoning and performance on the Wason Selection Task, Personality and Social Psychology Bulletin, 28, 1379-1387.

Ditto, P. H., \& Lopez, D. F. (1992). Motivated skepticism: Use of differential decision criteria for preferred and nonpreferred conclusions. Journal of Personality and Social Psychology, 63, 568-584.

Doherty, M. E., Mynatt, C. R., Tweney, R. D., \& Schiavo, M. D. (1979). Pseudodiagnosticity. Acta Psychologica, 43, 111-121.

Doherty, M. E., Schiavo, M. D., Tweney, R. D., \& Mynatt, C. R. (1981). The influence of feedback and diagnostic data on pseudodiagnositicity. Bulletin of the Psychonomic Society, 18, 191-194.

Edwards, W. (1968). Conservatism in human information processing. In B. Kleinmuntz (Ed.), Formal representation of human judgment. NewYork: Wiley.

Evans, J. St. B. T. (1993). The cognitive psychology of reasoning: An introduction. Quarterly Journal of Experimental Psychology Human Experimental Psychology, 46A, 561-567.

Evans, J. St. B. T. (1972). Interpretation and matching bias in a reasoning task. Quarterly Journal of Experimental Psychology, 24, 193-199.

Fairley, N., Manktelow, K., \& Over, D. (1999) Necessity, sufficiency, and perspective effects in causal conditional reasoning. Quarterly Journal of Experimental Psychology: Human Experimental Psychology, 52A, 771-790. 
Fiedler, K. (2000). Beware of samples! A cognitive-ecological sampling approach to judgment biases. Psychological Review, 107, 659-676.

Fischhoff, B., \& Beyth-Marom, R. (1983). Hypothesis evaluation from a Bayesian Perspective. Psychological Review, 90, 239-260.

Fischhoff, B, Bar-Hillel, M. (1984). Diagnosticity and the base-rate effect. Memory and Cognition, 12, 402-410.

Fitts, P.M., \& Posner, M. I. (1967). Human performance. Oxford, England: Brooks/Cole.

Frey, D., Schulz-Hardt, S., \& Stahlberg, D. (1996). Information seeking among individuals and groups and possible consequences for decision-making in business and politics. In E. Witte \& J. Davis (Eds.), Understanding group behavior: Vol. II. Small group processes and interpersonal relations (pp. 211-225). Mahwah, NJ: Erlbaum.

Frey, D. (1986). Recent research on selective exposure to information. In L. Berkowitz (Ed.), Advances in experimental social psychology (Vol. 19, pp. 41-80). New York: Academic Press.

Galotti, K., M., Baron, J., \& Sabini, J. P. (1986). Individual differences in syllogistic reasoning: Deduction rules or mental models? Journal of Experimental Psychology: General, 115, 16-25.

Gaeth, G. J., \& Shanteau, J. (1984). Reducing the influence of irrelevant information on experienced decision makers. Organizational Behavior and Human Performance, 33, 263-282. 
Gigerenzer, G., Hell, W., \& Blank, H. (1988). Presentation and content: The use of base rates as a continuous variable. Journal of Experimental Psychology: Human Perception and Performance, 14, 513-525.

Gigerenzer, G. (1996). On narrow norms and vague heuristics: A reply to Kahneman and Tversky. Psychological Review. 103, 592-596.

Hilton, D. J., \& Slugoski, B. R. (1986). Knowledge-based causal attributions: The abnormal conditions focus model. Psychological Review, 93, 75-88.

Hoch, S. J. (1985) Counterfactual reasoning and accuracy in predicting personal events. Journal of Experimental Psychology, 11, 719-731.

Jenkins, H. M., \& Ward, W. C. (1965) Judgment of contingency between responses and outcomes. Psychological Monographs: General \& Applied. 79 (1, Whole No. 594), 17.

Johnston, L. (1996). Resisting change: Information-seeking and stereotype change. European Journal of Social Psychology, 26, 799-825.

Johnson-Laird, P. N., Legrenzi, P., \& Legrenzi, M. S. (1972). Reasoning and a sense of reality. British Journal of Psychology. 6, 395-400.

Jonas, E., Schulz-Hardt, S., Frey, D., \& Thelen, N. (2001). Confirmation bias in sequential information search after preliminary decisions: An expansion of dissonance theoretical research on selective exposure to information. Journal of Personality and Social Psychology, 80, 557-571.

Kahneman, D., \& Tversky, A. (1996). On the reality of cognitive illusions: A reply to Gigerenzer's critique. Psychological Review, 103, 582-591. 
References 208

Kareev, Y., \& Halberstadt, N. (1993). Evaluating negative tests and refutation in a rule discovery task. Quarterly Journal of Experimental Psychology Human Experimental Psychology, 46A, 715-727.

Kelley, H. H. (1950). The warm-cold variable in first impressions of persons. Journal of Personality, 18, 431-439.

Kemmelmeier, M. (2004). Separating the wheat from the chaff: Does discriminating between diagnostic and nondiagnostic information eliminate the dilution effect? Journal of Behavioral Decision Making, 17, 231-243.

Klar, Y. (1990). Linking structures and sensitivity to judgment-relevant information in statistical and logical reasoning tasks. Journal of Personality and Social Psychology, 59, 841-858.

Klayman, J. \& Ha, Y., (1989). Hypothesis testing in rule discovery: Strategy, structure, and content. Journal of Experimental Psychology: Learning, memory, and Cognition, 15, 596-604.

Klayman, J., \& Ha, Y. (1987). Confirmation, disconfirmation, and information in hypothesis testing. Psychological Review, 94, 211-228.

Lendaris, G. G., (1986). On systemness and the problem solver: tutorial comments. Systems, Man, and Cybernetics. IEEE, Vol. SMC-16, No.4.

Lord, C. G., Lepper, M. R., \& Preston, E. (1984). Considering the opposite: A corrective strategy for social judgment. Journal of Personality and Social Psychology, 47, 1231-1243. 
References 209

Lord, C. G., Ross, L., \& Lepper, M. R. (1979). Biased assimilation and attitude polarization: The effects of prior theories on subsequently considered evidence. Journal of Personality and Social Psychology, 37, 2098-2109.

Luce, M. F., Bettman, J. R., \& Payne, J. W. (1997). Choice processing in emotionally difficult decisions. Journal of Experimental Psychology: Learning, Memory, and Cognition, 23, 384-405.

Lundgren, S. R., \& Prislin, R. (1998). Motivated cognitive processing and attitude change. Personality and Social Psychology Bulletin, 24, 715-726.

Maggi, J., Butera, F., Leggrenzi, P., \& Mugny, G. (1998). Relevance of information and social influence in the Pseudodiagnosticity bias. Swiss Journal of Psychology, 57, 188-199.

Manktelow, K. I. (1999). Reasoning and thinking. Hove, UK: Psychology Press.

Manktelow, K. I., \& Over, D. E. (1991). Social roles and utilities in reasoning with deontic conditionals. Cognition, 39, 85-105.

Manktelow, K. I., \& Over, D. E. (1992). Utility and deontic reasoning: Some comments on Johnson-Laird and Byrne. Cognition, 43, 183-188.

Murphy, K. R., \& Myors, B. (2004). Statistical Power Analysis: A simple and general model for traditional and modern hypothesis tests. $2^{\text {nd }}$ Ed, Mahwah, New Jersey: Lawerence Earlbaum Associates.

Mynatt, C. R., Doherty, M. E., \& Sullivan, J. A. (1991). Data selection in a minimal hypothesis testing task. Acta Psychologica, 76, 293-305. 
Ofir, C. (1988). Pseudodiagnosticity in judgment under uncertainty. Organizational Behavior and Human Decision Process, 42, 343-363.

Ormerod, T. C., Manktelow, K. I., \& Jones, G. V. (1993). Reasoning with three types of conditional: Biases and mental models. Quarterly Journal of Experimental Psychology: Human Experimental Psychology, 46A, 561-567.

Over, D. E., Manktelow, K.I., \& Hadjichristidis, C. (2004). Conditions for the Acceptance of Deontic Conditionals. Canadian Journal of Experimental Psychology, 58, 96-105.

Nickerson, R. S. (1998). Confirmation Bias: A ubiquitous phenomenon in many guises. Review of General Psychology, 2, 175-220.

Pennington, N., \& Hastie, R. (1993). A theory of explanation-based decision making. In G. A. Klein, J. Orasanu, R. Calderwood, \& C. E. Zsambok (Eds.), Decision making in action: Models and methods (pp. 188-201). Norwood, NJ: Ablex Publishing Corp.

Perkins, D. N., Farady, M. \& Bushey, B. (1991) Everyday reasoning and the roots of intelligence. In J F. Voss, D. N. Perkins, \& J. W. Segal (Eds.), Informal reasoning and education (pp.83-106). Hillsdale, NJ: Earlbaum.

Peterson, C. R., \& Beach, L. R. (1967). Man as an intuitive statistician, Psychological Bulletin, 68, 29-46.

Phillips L.D., Edwards, W. (1966). Conservatism in simple probability inference task. Journal of Experimental Psychology, 72, 346-354. 
References 211

Pinkley, R. L., Griffith, T. L., \& Northcraft, G. B. (1995). “Fixed pie” a la mode: Information availability, information processing, and the negotiation of suboptimal agreements. Organizational Behavior and Human Decision Processes, 62, $101-112$.

Platt, R. D., \& Griggs, R. A. (1993) Facilitation in the Abstract selection task: The effects of attention and instructional factors. Quarterly Journal of Experimental Psychology Human Experimental Psychology, 46A, 591-613.

Poletiek, F. H. (1996). Paradoxes of falsification. Quarterly Journal of Experimental Psychology: Human Experimental Psychology. 49A, 447-462.

Rudksi, J. M. (2002). Hindsight and confirmation biases in an exercise in telepathy. Psychological Reports, 91, 899-906.

Russo, E. J., Medvec, V. H., \& Meloy, M. G., (1996). The distortion of information during decisions. Organizational Behavior and Human Decision Processes, 66, 102-110.

Schulz-Hardt, S., Frey, D., Lüthgens, C., \& Moscovici, S. (2000). Biased information search in group decision making. Journal of Personality and Social Psychology, 78, 655-669.

Simon, D., Pham, L. B., Le, Q. A., \& Holyoak, K. J. (2001). The emergence of coherence over the course of decision making. Journal of Experimental Psychology: Learning, Memory, and Cognition, 27, 1250-1260.

Simon, H. (1997). The sciences of the artificial, $3^{\text {rd }}$ ed., MIT Press, Cambridge, Ma. 
Shaklee, H., \& Goldston, D. (1989). Development in causal reasoning: Information sampling and judgment rule. Cognitive Development, 4, 269-281.

Shaklee, H., \& Hall, L. (1983). Methods of assessing strategies for judging covariation between events. Journal of Educational Psychology, 75, 583-594.

Shaklee H., \& Tucker, D. (1980). A rule analysis of judgments of covariation between events. Memory and Cognition, 8, 459-467.

Shaklee H., \& Mims, M. (1982). Sources of error in judging events covariations. Journal of Experiment Psychology: Learning, Memory, and Cognition, 8, 208-224.

Shanteau, J. (1975). Averaging versus multiplying combination rules of inference judgment. Acta Psychologica, 39, 83-89.

Skov, R. B., \& Sherman, S. J. (1986). Information-Gathering processes: Diagnosticity, hypothesis-confirmatory strategies, and perceived hypothesis confirmation. Journal of Experimental Social Psychology, 22, 93-121.

Smedslund, J. (1963). The concept of correlation in adults. Scandinavian Journal of Psychology, 4, 544-551.

Smith, E.E., Shoben, E.J., \& Rips, L.J. (1974). Structure and process in semantic memory. Psychological Review, 81, 214-241.

Spellman, B. A., \& Mandel, D. R. (1999). When possibility informs reality: Counterfactual thinking as a cue to causality. Current Directions in Psychological Science, 8, 120-123. 
References 213

Tierney, J. (1991, July 21). Behind Monty Hall's Doors: Puzzle, Debate and Answer? The New York Times, p. A1.

Trautman, C. M., \& Shanteau, J. (1997). Inferences based on nondiagnostic information. Organizational Behavior and Human Decision Performance, 19, 43-55.

Trope, Y., \& Bassok, M. (1982). Confirmatory and diagnosing strategies in social information gathering. Journal of personality and social psychology, 43, 22-34.

Viswesvaran, C., \& Ones, D. S. (1999). Meta-Analyses of Fakability Estimates: Implications for Personality Measurement. Educational and Psychological Measurement, 59, 197-210.

Ward, W. C., \& Jenkins, H. M. (1965). The display of information and the judgment of contingency. Canadian Journal of Psychology. 19, 231-241.

Wason, P. C. (1966). Reasoning. In B. M. Foss (Ed.), New horizons in psychology I, Harmondsworth, Middlesex, England: Penguin.

Wason, P. C., \& Johnson-Laird, P.N. (1972). Psychology of reasoning, structure and content. Cambridge, Massachusetts: Harvard University Press.

Wason, P. C., \& Johnson-Laird, P. N. (1970). A conflict between selecting and evaluating information on an inferential task. British Journal of Psychology, 61, 509515. 
Appendices 214

\section{Appendix A}

Experimental Script.

\section{1 Attention}

\section{Balanced}

Imagine you are an archeologist specializing in South Sea cultures. Your research has led you to believe that the legendary Urn of Zor actually existed and was created on either Shell Island or nearby Coral Island, two islands that share a unique culture. You have found this Urn of Zor, resting on the ocean floor between Shell and Coral Islands.

Of the 10 urns that have previously been found at this spot on the ocean floor, 5 have been determined to have been made on Shell Island and 5 on Coral Island. Since there are no other islands with the same culture as Shell and Coral, no other island of origin is possible.

\section{Focused}

Imagine you are an archeologist specializing in South Sea cultures. Your research has led you to believe that the legendary Urn of Zor actually existed. While conducting underwater excavations, from your research facility on Shell Island, you have found this Urn of Zor, resting on the ocean floor.

\section{$\mathrm{IV}_{2}$ Motivation}

\section{Not Motivated}

The Urn of Zor is believed to have been created soon after the first settlements on the islands. Finding the Urn provides new information about the early colonization of the islands and will trigger new interest and research opportunities for you and your fellow South Sea archeologists and greatly enhance your prestige. This is especially important to you, since you are coming up for promotion and tenure next year.

\section{Motivated}

If it could be established that this urn had come from Shell Island, this would dispute the legend that Shell Island was the last island in the South Sea area to be settled. This would provide abundant research opportunities for you and your fellow South Sea archeologists and greatly enhance your prestige. This is especially important to you, since you are coming up for promotion and tenure next year. 
Appendices 215

\section{1 Attention}

\section{Balanced}

You need to make a determination as to the island on which the urn was more probably made. Your first step is to analyze the mineral content of the Urn of Zor. You find that the Urn of Zor has high content of the 8 minerals listed in the table below. Your second step is to determine the content of these minerals in the clay pits now in use on the two islands. The table below displays the results of the mineral content of the Urn of Zor and that of the current clay pits on the two islands:

\begin{tabular}{|l|l|}
\hline $\begin{array}{l}\text { Mineral found to be in } \\
\text { abundance in the Urn of } \\
\text { Zor }\end{array}$ & $\begin{array}{l}\text { Island whose current clay pits are } \\
\text { characterized by an abundance of this mineral }\end{array}$ \\
\hline Aluminum & Shell Island \\
\hline Calcium & Shell Island \\
\hline Chromium & Shell Island \\
\hline Copper & Shell Island \\
\hline Iron & Coral Island \\
\hline Magnesium & Coral Island \\
\hline Nickel & Coral Island \\
\hline Zinc & Coral Island \\
\hline
\end{tabular}

Unfortunately, the islanders have migrated around the islands over the years and have changed the clay pits they use. While the mineral content of the current clay pits is probably similar to the mineral content of those clay pits used in ancient times to make the Urn of Zor, it is certainly not identical. Therefore you will need an additional step in your analysis.

At this point what is the probability the urn came from:

Shell Island
Coral Island ?

\section{Focused}

When you return to your research facility on Shell Island, your first thought is to analyze the mineral content of the Urn of Zor. The results indicate that the Urn of Zor has a high content of eight minerals (Aluminum, Calcium, Chromium, Copper, Iron, Magnesium, Nickel and Zinc). Four (Aluminum, Calcium, Chromium and Copper) of these high content minerals are characteristic of the clay pits in current use on Shell Island. When you tell your research team the results, one of your co-workers states that some of these minerals remind him of the current clay pit on the side of Shell Island he has been working on. A second co-worker states that some of these minerals remind her of the current clay pit on another side of Shell Island on which she has been working. This sets off a furious debate among your research team as to the side of Shell Island on which the Urn was more probably made. Unfortunately, the evidence is not conclusive because, the islanders have migrated around the islands 
over the years and have changed the clay pits they use. While the mineral content of the current clay pits is probably similar to the mineral content of those clay pits used in ancient times to make the Urn of Zor, it is certainly not identical. Therefore you will need an additional step in your analysis.

At this point what is the probability the Urn came from anywhere on Shell Island ?

$I V_{2}$ Motivation

1 Not Motivated

(No manipulation check)

\section{Motivated}

Would it be good for your career if the probability were higher or lower that the Urn came from Shell Island?

\section{1 Attention}

\section{Balanced}

(Nothing)

\section{Focused}

You announce the finding of the Urn of Zor and your intention to give it to the Shell Island National Museum, which you believe to be the most appropriate representative of the rightful owners. Coral Island, which has the same culture as Shell Island, quickly requests that you investigate the possibility that the Urn might have been made on their island, and requests that you not give the Urn to the Shell Island National Museum until you confirm that the Urn came from Shell Island.

Coral Island argues that of the 10 urns that have previously been found at the spot on the ocean floor where the Urn of Zor was found, 5 have been determined to have been made on Shell Island and 5 on Coral Island. Since there are no other islands with the same culture as Shell and Coral, no other island of origin is possible.

Additionally, they argue that despite the fact that four of the minerals (Aluminum, Calcium, Chromium and Copper) characterize the current clay pits of Shell Island, the other four minerals (Iron, Magnesium, Nickel and Zinc) characterize the current clay pits of Coral Island.

(These two pages are page 2) 
Appendices 217

\section{All Conditions}

Since the evidence you have collected so far is not definitive as to which island the Urn of Zor was made on, you decide to take the additional step of comparing the mineral content of the Urn of Zor with the mineral content of urns made at the same time as the Urn of Zor. A private collector owns all the urns from that era whose island of origin has been clearly established (20 urns known to have been made on Shell Island and 20 known to have been made on Coral Island).

This private collector, in order to limit damage to his urns, requires you to remove only enough material from his urns to perform 4 of the 16 possible mineral tests. Each test is specific to a mineral and an island. Each test would tell you the number of the 20 urns from the specified island that have the same high content of the specified mineral as that in the Urn of Zor.

Choose 4 of the following 16 tests to perform, as the most efficient way to determine the origin of the Urn of Zor, following the instructions below.

\section{$\mathrm{IV}_{3}$ Timing}

\section{Sequential}

Put the number 1 next to the first test you would like to perform, and then remove the corresponding sticker to reveal the test result.

Next choose your second test, put the number 2 next to that test and then remove the corresponding sticker to reveal the test result.

Next choose your third test, put the number 3 next to that test and then remove the corresponding sticker to reveal the test result.

Finally, choose your fourth test, put the number 4 next to that test and then remove the corresponding sticker to reveal the test result.

\section{Simultaneous}

Put a number (1 to 4 ) next to the four tests you would like to perform in the order of importance to you ( 1 being top priority, 4 being lowest). Then remove the four corresponding stickers to reveal the four test results. 
Analysis of the urns made at the same time as the Urn of Zor

\begin{tabular}{|c|c|c|c|c|c|}
\hline \multirow[t]{2}{*}{$\begin{array}{l}\text { Mineral found } \\
\text { to be in } \\
\text { abundance in } \\
\text { the Urn of Zor } \\
\text { Aluminum }\end{array}$} & \multirow{2}{*}{$\begin{array}{l}\begin{array}{l}\text { Characterizes } \\
\text { the current } \\
\text { clay pits of }\end{array} \\
\text { Shell Island }\end{array}$} & \multicolumn{2}{|c|}{$\begin{array}{l}\text { Number of the } 20 \text { old urns } \\
\text { from Shell Island that } \\
\text { have an abundance of this } \\
\text { mineral }\end{array}$} & \multicolumn{2}{|c|}{$\begin{array}{l}\text { Number of the } 20 \text { old urns } \\
\text { from Coral Island that } \\
\text { have an abundance of this } \\
\text { mineral }\end{array}$} \\
\hline & & 2 or 12 & Choice \# & 1 or 6 & Choice \# \\
\hline Calcium & Shell Island & 8 or 18 & Choice \# & 4 or 9 & Choice \# \\
\hline Chromium & Shell Island & 6 or 16 & Choice \# & 3 or 8 & Choice \# \\
\hline Copper & Shell Island & 4 or 14 & Choice \# & 2 or 7 & Choice \# \\
\hline Iron & Coral Island & 2 or 7 & Choice \# & 4 or 14 & Choice \# \\
\hline Magnesium & Coral Island & 3 or 8 & Choice \# & 6 or 16 & Choice \# \\
\hline Nickel & Coral Island & 4 or 9 & Choice \# & 8 or 18 & Choice \# \\
\hline Zinc & Coral Island & 1 or 6 & Choice \# & 2 or 12 & Choice \# \\
\hline
\end{tabular}

The actual \#s are covered by stickers the numbers are IV 4 (see below)

\section{$\mathrm{IV}_{4}$ Conditionals:}

1 Low

$p(\mathrm{~d} \mid \mathrm{h} 2) 20 \%$ - 10\% (2-1, 4-2, 6-3, 8-4)

2 High

$p(\mathrm{~d} \mid \mathrm{h} 1)$ 80\% - 40\% (18-9, 16-8, 14-7, 12-6)

\section{All Conditions}

Which Island do you now believe the Urn of Zor is more likely to have come from?

What do you now believe is the probability that the Urn of Zor came from that island? $\%$

What do you believe is the probability that the Urn of Zor came from the other island? $\%$

(These two pages will be page 3 of the script) 
Appendices 219

\section{All Conditions}

What information did you use in making this estimate of the probability of the island of origin? Put a check on the right next to all the pieces of information below that you used.

\section{Location the Urn was Found}

Of the 10 urns that have previously been found at this spot of the ocean floor 5 have been determined to have been made on Shell Island and the other 5 on Coral Island.

\section{Current Clay Pit Information}

Mineral Tested that the

Urn of Zor had High content

Aluminum

Calcium

Chromium

Copper

Iron

Magnesium

Nickel

Zinc
This mineral content characterizes the current clay pits of

Shell Island

Shell Island

Shell Island

Shell Island

Coral Island

Coral Island

Coral Island

Coral Island

\section{Old Urns Information}

Your first choice of the mineral tests on the old urns.

Your second choice of the mineral tests on the old urns.

Your third choice of the mineral tests on the old urns.

Your fourth choice of the mineral tests on the old urns.

\section{Other Information}

Please describe

(This would be page 4 of the script) 
Appendices 220

\section{All Conditions}

If you used indicated on the previous page that you used one piece of evidence, then go to question 3 below.

If you used indicated on the previous page that you used more than one piece of evidence, then go to question 1 below.

1) Tell me how you went about making your estimate of the probability that the Urn came from the island you chose. You can use a verbal description or a formula to describe the method you used.

2) Place a check next to all the mathematical processes you used? (Check all that apply)

Type of Method

Addition

Subtraction

Multiplication

Division

Averaging

Other
Mathematical Example using two pieces of evidence (evidence \#1 + evidence \#2)

(evidence \#1 - evidence \#2)

(evidence \#1 * evidence \#2)

(evidence \#1 / evidence \#2)

(evidence \#1 + evidence \#2)/ 2

3) Did you find the results of any of the tests on the old urns surprising? (Yes / No)

4) If yes, indicate which ones and whether they were higher or lower than expected.

(Please look back to see your four selections on page three and then circle higher or lower below).

$\begin{array}{llr}\text { First Choice Surprising } & \text { higher } & \text { lower } \\ \text { Second Choice Surprising } & \text { higher } & \text { lower } \\ \text { Third Choice Surprising } & \text { higher } & \text { lower } \\ \text { Fourth Choice Surprising } & \text { higher } & \text { lower }\end{array}$

5) Please go back to page three again and, without removing any more stickers, write down on the stickers what you think the value of the tests would be that you did not choose to perform. 
Appendices 221

\section{All Conditions}

6) Circle one or more of the following racial / ethnic groups that best applies to you. African American

Caucasian

Latino / Hispanic

Asian / Pacific Islander

Native American

Other

7) Circle your gender. Male $\quad$ Female

8) What is the number of college level statistics classes you have completed?

9) What is your college G.P.A. (on a 4 point scale $4=A$, 3=B etc.) 
Appendices 222

\section{Appendix B}

\section{Instructions.}

Participants will be told to read the experimental instructions and to raise their hands when they have a questions or when they have completed the experiment. They will be told not to talk to or look at their neighbors papers. 
Appendices 223

\section{Appendix C}

Demographic Variables.

1) Race (African American, Caucasian, Latino / Hispanic, Asian / Pacific Islander, Native American, Other)

2) Gender ～(Male, Female)

3) Number of college level statistics classes completed.

4) What is your college G.P.A. 
Appendices 224

\section{Appendix D}

Informed Consent Form.

\section{Decision Making Study}

You are invited to participate in a research study conducted by Geoffrey Borthwick from Portland State University, Psychology. The researcher hopes to learn how people make decisions in partial fulfillment of the requirements for a doctoral degree, under the supervision of Barry Anderson. You were selected as a possible participant in this study because you because you have not participated in this study previously.

If you decide to participate, you will be asked to read a scenario and answer hypothetical questions in it, including making decision and asking you about how you made those decisions. The study will take approximately 30 minutes. While participating in this study you may feel uncomfortable in answering these questions. You may receive extra credit from the instructor in the class from which you were recruited.

Any information that is obtained in connection with this study and that can be linked to you or identify you will be kept confidential, except the possibility of reporting your participation to your instructor solely for the purpose of your obtaining extra credit in that class. This information will be kept confidential by separating it from your name and storing all materials in a locked room.

Your participation is voluntary. You do not have to take part in this study, and it will not affect your relationship with Portland State University. You may also withdraw from this study at any time without affecting your relationship with Portland State University.

If you have any concerns or problems about your participation in this study or your rights as a research subject, please contact the Human Subjects Research Review Committee, Office of Research and Sponsored Projects, 111 Cramer Hall, Portland State University, (503) 725-4288. If you have any questions about the study itself, contact Geoffrey Borthwick at borthwg@pdx.edu or (503) 725-3989.

Your signature indicates that you have read and understand the above information and agree to take part in this study. Please understand that you may withdraw your consent at any time without penalty, and that, by signing, you are not waiving any legal claim, rights or remedies. The researcher will provide you with a copy of this form for your own records. 
Appendices 225

\section{Appendix E}

\section{Pilot Study Integration Strategies.}

Integration Strategies found in pilot study, and the number (and percentages) of subjects who used each (Borthwick \& Anderson, 2001).

\begin{tabular}{|c|c|c|}
\hline Strategy (Numbered as in the present study) & $\begin{array}{l}\text { Number of } \\
\text { participants }\end{array}$ & Percentage \\
\hline 1 Bayesian & 0 & $0 \%$ \\
\hline 2 Highest probability conditioned on focal island & \multirow{2}{*}{$\begin{array}{l}5 \text { (Sub-strategies not } \\
\text { distinguished in pilot } \\
\text { study) }\end{array}$} & \multirow[t]{2}{*}{$9.4 \%$} \\
\hline $\begin{array}{l}11 \text { Highest probability, conditioned on and } \\
\text { expected to favor focal Island }\end{array}$ & & \\
\hline 3 Average of all available conditional probabilities & 5 & $9.4 \%$ \\
\hline $\begin{array}{l}4 \text { Average of the probabilities conditioned on focal } \\
\text { island }\end{array}$ & 5 & $9.4 \%$ \\
\hline $\begin{array}{l}5 \text { Average of the base rate and the probabilities } \\
\text { conditioned on focal island }\end{array}$ & 1 & $1.9 \%$ \\
\hline $\begin{array}{l}6 \text { Sum of all probabilities conditioned on focal } \\
\text { island }\end{array}$ & \multirow{3}{*}{$\begin{array}{l}24 \text { (Sub-strategies } \\
\text { not distinguished in } \\
\text { pilot study) }\end{array}$} & \multirow[t]{3}{*}{$45.3 \%$} \\
\hline $\begin{array}{l}7 \text { Sum of all probabilities conditioned on focal } \\
\text { island, minus the sum of all probabilities } \\
\text { conditioned on the non-focal island }\end{array}$ & & \\
\hline $\begin{array}{l}8 \text { Sum of all probabilities conditioned on focal } \\
\text { island plus the base rate, minus the sum of all } \\
\text { probabilities conditioned on the non-focal island }\end{array}$ & & \\
\hline 9 Base Rate & 6 & $11.3 \%$ \\
\hline 10 Even (50\%-50\%) & 2 & $3.8 \%$ \\
\hline 12 Base Rate with non-numeric adjustment & 5 & $9.4 \%$ \\
\hline
\end{tabular}

